\title{
Endogenous RNAi pathways in $C$. elegans
}

\author{
Allison C. Billi ${ }^{1}$, Sylvia E. J. Fischer ${ }^{2}$, and John K. Kim ${ }^{1 \S}$ \\ ${ }^{1}$ Life Sciences Institute, Department of Human Genetics, University of Michigan, Ann Arbor MI, \\ USA \\ ${ }^{2}$ Department of Molecular Biology, Massachusetts General Hospital, Department of Genetics, \\ Harvard Medical School, Boston MA, USA
}

\section{Table of Contents}

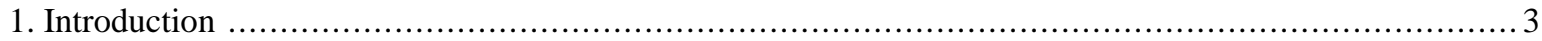

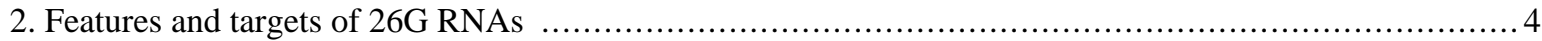

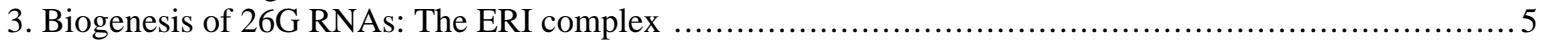

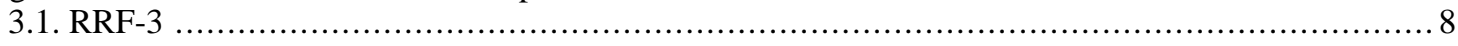

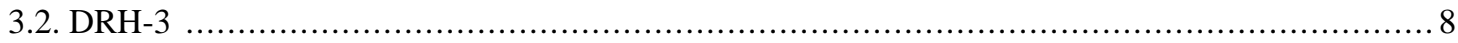

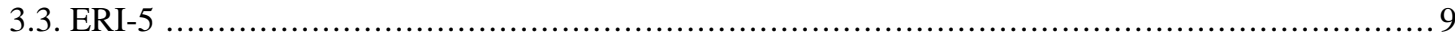

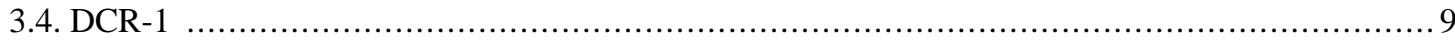

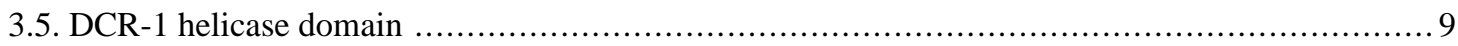

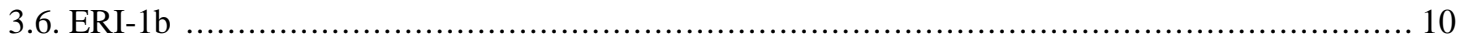

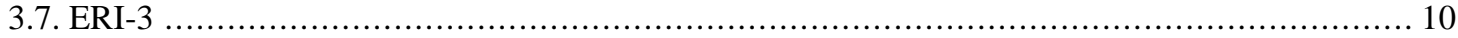

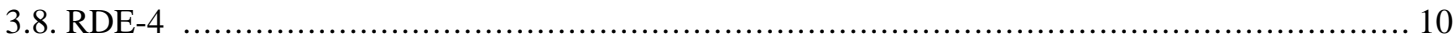

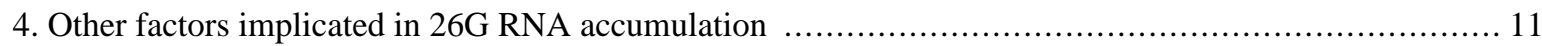

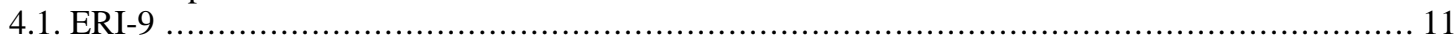

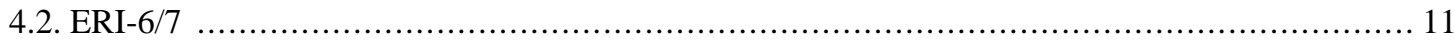

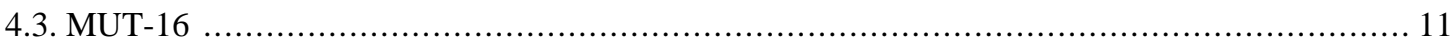

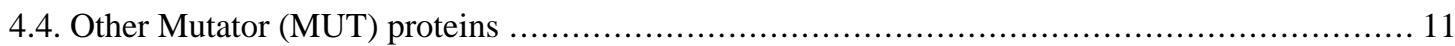

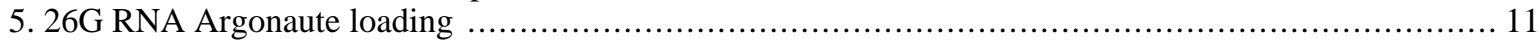

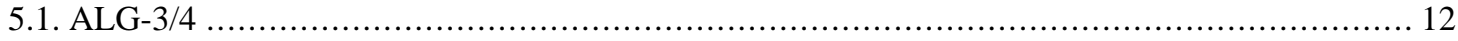

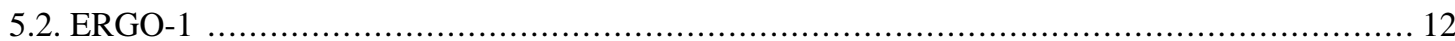

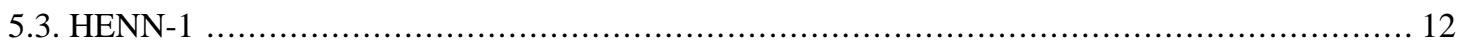

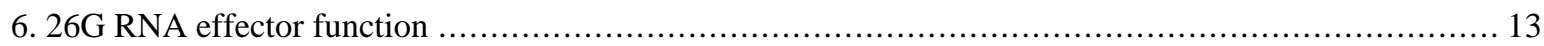

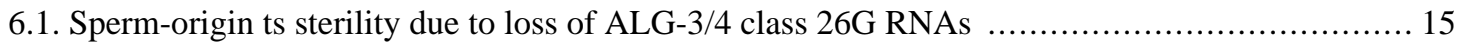

6.2. Enhanced RNAi sensitivity due to loss of ERGO-1 class $26 \mathrm{G}$ RNAs ........................... 16

\footnotetext{
*Edited by Julie Ahringer. Last revised Novermber 10, 2013. Published May 7, 2014. This chapter should be cited as: Billi et al. Endogenous RNAi pathways in C. elegans (May 7, 2014), WormBook, ed. The C. elegans Research Community, WormBook, doi/10.1895/wormbook.1.170.1, http://www.wormbook.org.

Copyright: ( 2014 Allison C. Billi, Sylvia E. J. Fischer, John K. Kim. This is an open-access article distributed under the terms of the Creative Commons Attribution License, which permits unrestricted use, distribution, and reproduction in any medium, provided the original author and source are credited.

${ }^{\S}$ To whom correspondence should be addressed. E-mail: jnkim@umich.edu
} 
7. 22G RNAs comprise two major small RNA pathways ....................................................... 16

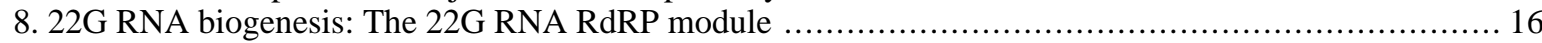

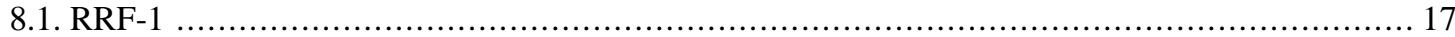

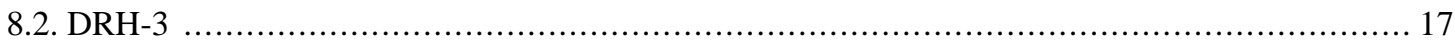

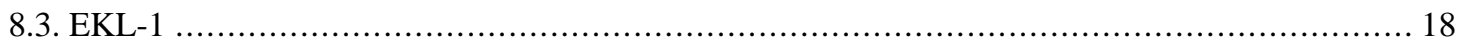

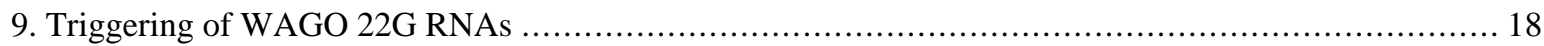

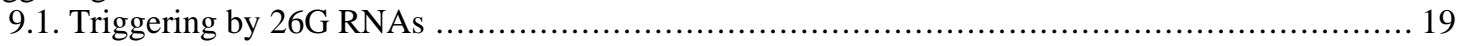

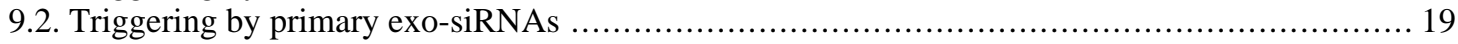

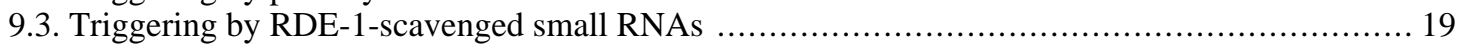

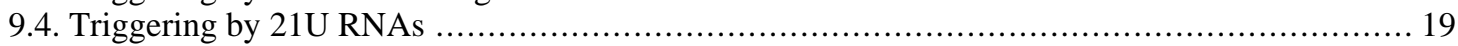

10. Germline amplification of WAGO 22G RNAs in perinuclear Mutator foci ............................. 20

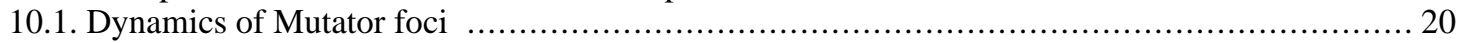

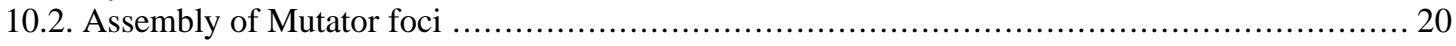

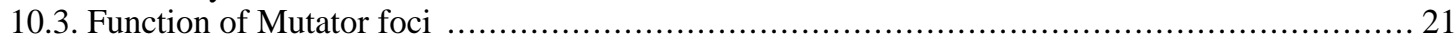

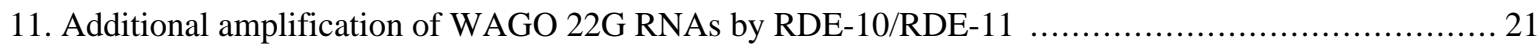

11.1. RDE-10/RDE-11 amplifies 22G RNAs triggered by exo-RNAi and ERGO-1 ....................... 21

11.2. RDE-10/RDE-11-mediated target degradation ...................................................... 22

11.3. RSD-2, RSD-6, and/or HAF-6 may potentiate RDE-10/RDE-11 function ......................... 22

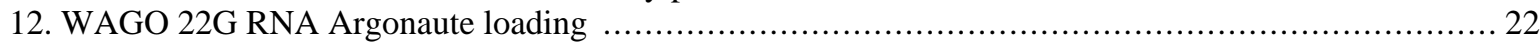

13. WAGO 22G RNA-mediated post-transcriptional gene silencing ....................................... 23

14. WAGO 22G RNA-mediated transcriptional gene silencing: The nuclear RNAi pathway .................. 24

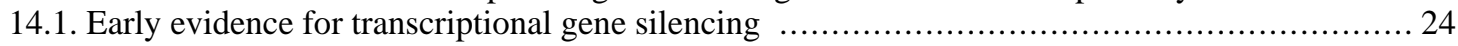

14.2. Early evidence for heritable and multigenerational gene silencing ................................ 24

14.3. NRDE-3 and HRDE-1 WAGO Argonautes mediate nuclear RNAi ............................... 24

14.4. NRDE-3 collaborates with other WAGO Argonautes to silence somatic targets .................... 25

14.5. HRDE-1 transgenerationally silences diverse targets to promote germline immortality ............25

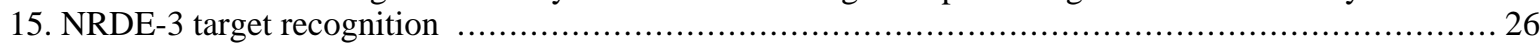

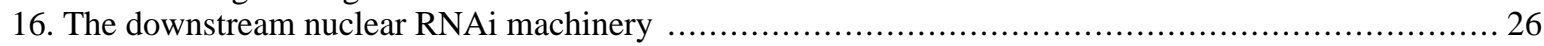

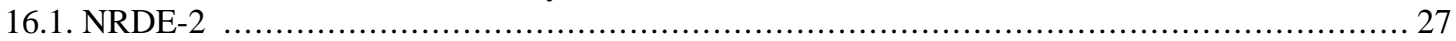

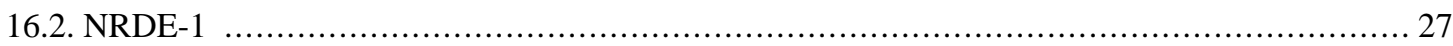

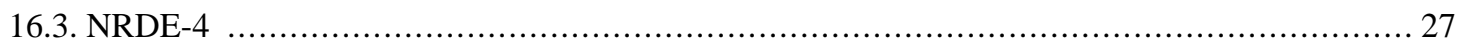

17. Nuclear RNAi inhibits transcriptional elongation and drives H3K9 trimethylation ........................ 27

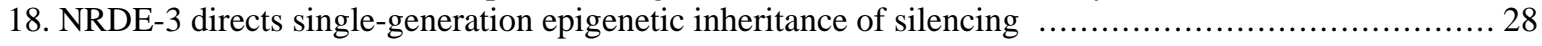

19. HRDE-1 directs multigenerational epigenetic inheritance of silencing .................................. 29

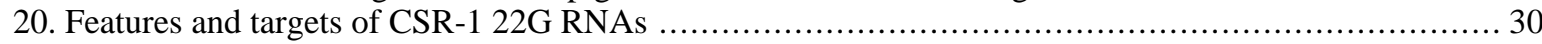

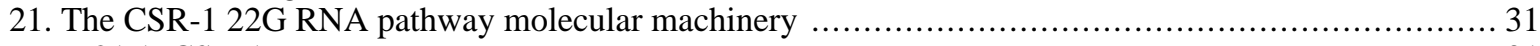

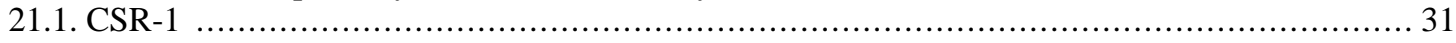

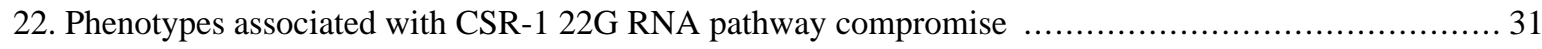

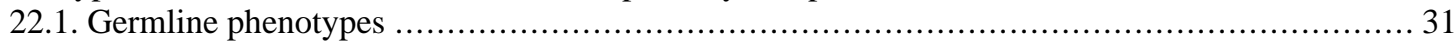

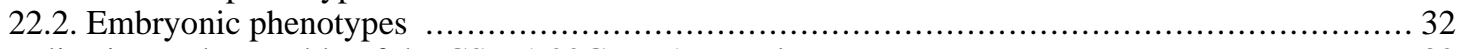

23. Localization and assembly of the CSR-1 22G RNA complex …...................................... 32

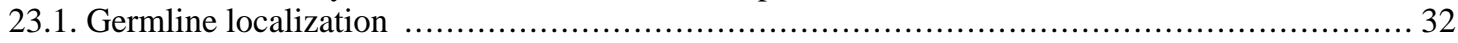

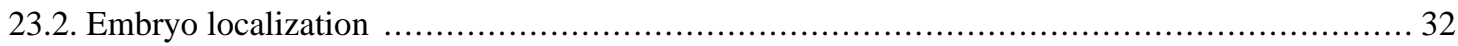

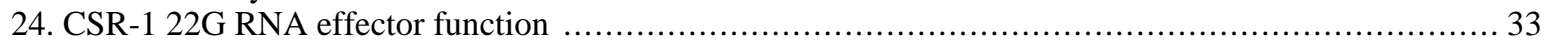

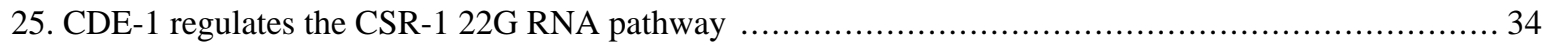

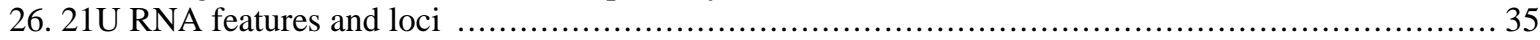

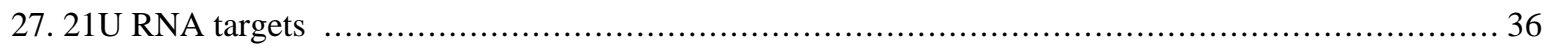

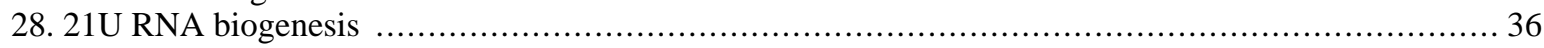

28.1. The 21U RNA large motif and Forkhead family transcription factors ............................. 37

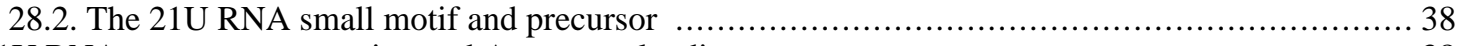

29. 21U RNA precursor processing and Argonaute loading ….............................................. 38

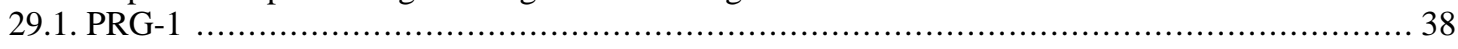

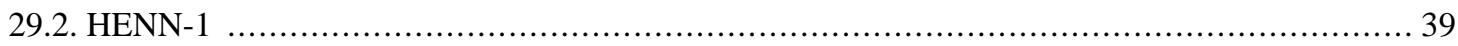

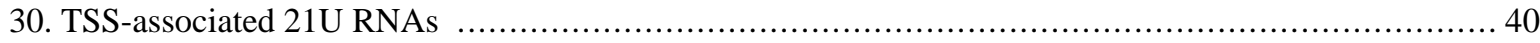

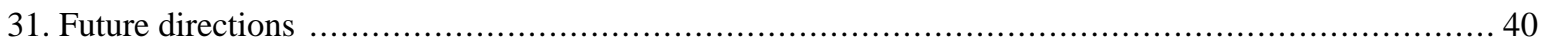

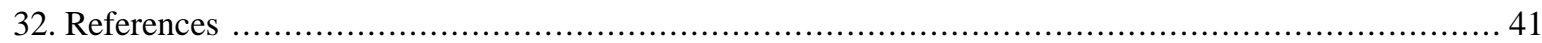


Abstract

In addition to several hundred microRNAs, $C$. elegans produces thousands of other small RNAs targeting coding genes, pseudogenes, transposons, and other noncoding RNAs. Here we review what is currently known about these endogenous small interfering RNAs (siRNAs) and piwi-interacting RNAs (piRNAs), providing an overview of their biogenesis, their associated protein factors, and their effects on mRNA dynamics and chromatin structure. Additionally, we describe how the molecular actions of these classes of endogenous small RNAs connect to their physiological roles in the organism.

\section{Introduction}

The phenomenon of RNA interference (RNAi), wherein introduction of dsRNA results in silencing of homologous genes, was first observed and reported in C. elegans. In addition, when the first small RNA, the lin-4 microRNA, was identified, it was thought to be a curiosity of $C$. elegans biology until the discovery of the conserved let-7 family of microRNAs. Since then, thousands of unique endogenous small RNAs have been found in C. elegans falling into three distinct classes: microRNAs, endogenous small interfering RNAs (endo-siRNAs), and Piwi-interacting RNAs (piRNAs). All three types of small RNAs bind to Argonaute effector proteins, recognize target transcripts exhibiting partial or perfect complementarity, and direct target regulation that is primarily inhibitory in nature. Yet microRNAs, endo-siRNAs, and piRNAs vary greatly in biogenesis mechanisms, specific protein cofactors, and even effector function. The details of $C$. elegans microRNA biology have been previously described (Fischer, 2010) and are addressed in this review only insofar as they intersect with endo-siRNA and piRNA biology. Similarly, a thorough examination of exogenously-derived siRNAs and exogenous RNAi (exo-RNAi), and its function as an anti-viral silencing response, is beyond the scope of this review; however, the gene silencing mechanisms initiated by exo-RNAi are discussed, as C. elegans exo-RNAi engages a downstream endo-RNAi amplification pathway also shared by primary endo-siRNAs and piRNAs.

The gene silencing capacity of antisense RNA was first described in C. elegans over two decades ago (Fire et al., 1991). The effective interfering agent was subsequently determined to be double-stranded RNA (dsRNA), and its incredible potency suggested the existence of a catalytic or amplification mechanism engaged by exogenous dsRNA (Fire et al., 1998). This dsRNA is processed into primary exo-siRNAs by the conserved RNase III enzyme Dicer (Zamore et al., 2000; Bernstein et al., 2001), increasing the ratio of trigger to target, but still to a degree insufficient to explain the potency of exo-RNAi. Further studies of the small RNA effector populations during exo-RNAi in C. elegans revealed that primary exo-siRNAs are not the ultimate effectors of interference; rather, they trigger vigorous production of secondary siRNAs by RNA-dependent RNA polymerases (RdRPs), amplifying the signal and focusing the interference response on expressed sequences (Sijen et al., 2001). Before the discovery of endogenous silencing pathways in $C$. elegans, isolation of mutations causing deficiencies in both exo-RNAi and endogenous silencing phenomena such as transposon silencing suggested a shared mechanism (Tabara et al., 1999; Ketting et al., 1999; Ketting and Plasterk, 2000). Mutations in core molecular machinery of RNAi also were found to result in phenotypes indicating essential endogenous roles. Loss of DCR-1, the sole C. elegans ortholog of Dicer, results in profound cell fate specification defects and germline abnormalities leading to lethality and sterility (Grishok et al., 2001; Ketting et al., 2001; Knight and Bass, 2001); the RdRP EGO-1 is required for germline development (Smardon et al., 2000); and mutants defective in transposon silencing and RNAi show compromised fertility (Ketting et al., 1999; Tijsterman et al., 2002a; Vastenhouw et al., 2003; Chen et al., 2005).

The discovery of the first microRNA, lin-4, in C. elegans suggested that endogenous small RNAs also initiate transcript regulation through antisense mechanisms (Lee et al., 1993; Wightman et al., 1993). After the identification of a second microRNA, let-7, in 2000 (Reinhart et al., 2000), cloning approaches were successfully applied to expand dramatically the repertoire of microRNAs in C. elegans (Lau et al., 2001; Lee and Ambrose 2001). It was not until a decade after the discovery of the first microRNA that other endogenous small RNAs were identified in $C$. elegans (Ambros et al., 2003; Lim et al., 2003), and only in 2006 did small RNA deep sequencing first reveal the incredible diversity of C. elegans small RNAs (Ruby et al., 2006). Among the species identified were a large pool of 5' guanosine antisense small RNAs identified as endo-siRNAs that appeared to represent distinct 26- and 22-nucleotide (nt) subpopulations, later determined to correspond to primary and secondary endo-siRNAs, respectively (Ruby et al., 2006; Pak and Fire, 2007; Sijen et al., 2007; Han et al., 2009; Conine et al., 2010; Vasale et al., 2010). Subsequent dissection of these $26 \mathrm{G}$ and $22 \mathrm{G}$ RNA populations identified unique subgroups with largely overlapping biogenesis requirements but that engage different effector pathways distinguished by the particular Argonaute protein(s) interacting with the small RNAs. C. elegans encodes 27 Argonaute proteins. Roughly half of these proteins belong to a worm-specific Argonaute (WAGO) clade and bind 22G RNAs of 
particular subclasses, the WAGO 22G RNAs and CSR-1 22G RNAs; the former represent secondary siRNAs that effect target silencing, whereas the latter are a class of siRNAs complementary to germline-expressed transcripts that do not silence target genes but rather promote proper chromosome organization during mitosis (Claycomb et al., 2009; Gu et al., 2009). Also identified in the initial deep sequencing dataset were the 21U RNAs, 5' uridine 21-nt small RNAs later determined to represent the piRNAs of C. elegans (Batista et al., 2008; Das et al., 2008). This review summarizes the literature contributing to our current understanding of the C. elegans 26G RNAs, WAGO 22G RNAs, CSR-1 22G RNAs, and 21U RNAs, discussing mechanisms of triggering, biogenesis, and effector function, where known. Whereas microRNAs are required for diverse developmental and physiological processes in the soma, endo-siRNAs and 21U RNAs serve as the guardians of the immortal germline, constituting a complex, interconnected, and tremendously robust system for surveillance of the C. elegans genome.

\section{Features and targets of 26G RNAs}

Early deep sequencing of $C$. elegans small RNAs revealed a distinct population of 26 nt species that are largely anti-sense to annotated genes and therefore classified as endo-siRNAs (Ruby et al., 2006). Like endo-siRNAs sequenced earlier in C. elegans (Ambros et al., 2003), these 26 nt species show a 5' guanosine bias and are thus termed 26G RNAs (Ruby et al., 2006; Asikainen et al., 2008; Han et al., 2009; Stoeckius et al., 2009; Gent et al., 2010) (Table 1). 26G RNAs are 5' monophosphorylated (Ruby et al., 2006; Han et al., 2009; Stoeckius et al., 2009; Gent et al., 2010). 26G RNAs are quite enriched in male and female germlines (Han et al., 2009) where they comprise two distinct subpopulations that are temporally isolated and bound by unique, germline-specific effector complexes. 26G RNAs in the spermatogenic gonad are bound by redundant Argonautes ALG-3 and ALG-4, whereas 26G RNAs in oogenic gonad are bound by the ERGO-1 Argonaute (Han et al., 2009; Conine et al., 2010; Vasale et al., 2010). ERGO-1 class 26G RNAs are also highly abundant in embryos (Han et al., 2009; Stoeckius et al., 2009) and perdure through early larval development (Gent et al., 2010).

Table 1. Features of different classes of endogenous small RNAs.

\begin{tabular}{|c|c|c|c|c|c|c|}
\hline Small RNA & 5 ' terminus & 3' terminus & Polymerase & $\begin{array}{l}\text { Biogenesis } \\
\text { factors }\end{array}$ & \begin{tabular}{|l|} 
Cofactors \\
regulating \\
accumulation
\end{tabular} & $\begin{array}{l}\text { Argonaute(s) } \\
\text { and RISC } \\
\text { components }\end{array}$ \\
\hline $\begin{array}{l}\text { ERGO-1 class } \\
\text { 26G RNA }\end{array}$ & Monophosphorylated & 2'-O-methylated & RdRP RRF-3 & $\begin{array}{l}\text { DRH-3, } \\
\text { ERI-5, } \\
\text { DCR-1, } \\
\text { ERI-1b, } \\
\text { ERI-3, } \\
\text { RDE-4 }\end{array}$ & $\begin{array}{l}\text { HENN-1, } \\
\text { ERI-9, } \\
\text { ERI-6/7, } \\
\text { MUT-16 }\end{array}$ & ERGO-1 \\
\hline $\begin{array}{l}\text { ALG-3/4 class } \\
\text { 26G RNA }\end{array}$ & Monophosphorylated & Hydroxylated & RdRP RRF-3 & $\begin{array}{l}\text { DRH-3, } \\
\text { ERI-5, } \\
\text { DCR-1, } \\
\text { ERI-1b, } \\
\text { ERI-3, } \\
\text { RDE-4 }\end{array}$ & & ALG-3/4 \\
\hline $\begin{array}{l}\text { WAGO 22G } \\
\text { RNA }\end{array}$ & Triphosphorylated & Hydroxylated & $\begin{array}{l}\text { RdRPs } \\
\text { RRF-1/EGO- }\end{array}$ & $\begin{array}{l}\text { RRF-1/EGO- } \\
\text { DRH-3, } \\
\text { EKL-1 }\end{array}$ & $\begin{array}{l}\text { MUT-16, } \\
\text { MUT-7, } \\
\text { MUT-8, } \\
\text { MUT-2, } \\
\text { MUT-15, } \\
\text { MUT-14, } \\
\text { RDE-10, } \\
\text { RDE-11 }\end{array}$ & $\begin{array}{l}\text { WAGO } \\
\text { Argonautes }\end{array}$ \\
\hline $\begin{array}{l}\text { CSR-1 22G } \\
\text { RNA }\end{array}$ & Triphosphorylated & Hydroxylated & RdRP & EGO-1 & $\begin{array}{l}\text { EGO-1, } \\
\text { DRH-3, } \\
\text { EKL-1 }\end{array}$ & CDE-1 \\
\hline $\begin{array}{l}\text { 21U RNA } \\
\text { (Type I) }\end{array}$ & Monophosphorylated & 2'-O-methylated & RNA Pol II & $\begin{array}{l}\text { FKH-3/4/5, } \\
\text { UNC-130, } \\
\text { (large and } \\
\text { small motifs) }\end{array}$ & HENN-1 & PRG-1 \\
\hline
\end{tabular}




\begin{tabular}{|l|l|l|l|l|l|l|}
\hline Small RNA & $\mathbf{5}$, terminus & 3, terminus & Polymerase & $\begin{array}{l}\text { Cofactors } \\
\text { Biogenesis } \\
\text { factors }\end{array}$ & $\begin{array}{l}\text { Argonaute(s) } \\
\text { and RISC } \\
\text { regulating } \\
\text { accumulation } \\
\text { components }\end{array}$ \\
\hline $\begin{array}{l}\text { TSS-associated } \\
\text { 21U RNA } \\
\text { (Type II) }\end{array}$ & & & RNA Pol II & (Small motif) & & PRG-1 \\
\hline microRNA & Monophosphorylated & Hydroxylated & RNA Pol II & $\begin{array}{l}\text { DCR-1, } \\
\text { DRSH-1, } \\
\text { PASH-1 }\end{array}$ & $\begin{array}{l}\text { XRN-2, } \\
\text { DCS-1 }\end{array}$ & $\begin{array}{l}\text { ALG-1/2, } \\
\text { AIN-1/2, } \\
\text { NHL-2, } \\
\text { CGH-1, } \\
\text { TSN-1, } \\
\text { VIG-1 }\end{array}$ \\
\hline
\end{tabular}

Little is known about how transcripts are selected for targeting by 26G RNAs. 26G RNAs map primarily to protein-coding genes with a strong antisense bias, although some target unannotated loci (Ruby et al., 2006; Han et al., 2009; Stoeckius et al., 2009). They are transcribed from spliced mRNA templates by the RNA-dependent RNA polymerase RRF-3 (Gent et al., 2009; Han et al., 2009; Conine et al., 2010; Gent et al., 2010), as indicated by the sequencing of rare species spanning exon-exon junctions (Ruby et al., 2006; Han et al., 2009; Gent et al., 2010) and by the loss of complementary 26G RNAs upon nonsense-mediated decay of template mRNA (Han et al., 2009).

Targets of ALG-3/4 class 26G RNAs are highly enriched for transcripts classified as spermatogenesis-enriched (Reinke et al., 2004; Gent et al., 2009; Han et al., 2009; Conine et al., 2010), explaining earlier observations connecting endo-siRNAs primarily with regulation of sperm function genes (Ruby et al., 2006; Asikainen et al., 2007). ALG-3/4 class 26G RNAs map across the lengths of their target sequences, but preferentially target transcript 5' and 3' termini; transcripts exhibiting higher 5' UTR targeting appear to be more efficiently silenced (Conine et al., 2010).

Although biogenesis of ERGO-1 class 26G RNAs initiates in oogenesis, their targets are depleted of germline-intrinsic transcripts (Reinke et al., 2004; Han et al., 2009); accordingly, ERGO-1 class 26G RNAs primarily regulate zygotic targets throughout development (Han et al., 2009; Gent et al., 2010). The majority of abundant ERGO-1 class 26G RNAs map to within $5 \mathrm{Mb}$ of chromosome ends at more gene-poor regions (Vasale et al., 2010; Fischer et al., 2011). Unlike ALG-3/4 class 26G RNAs, ERGO-1 class 26G RNAs are excluded from the first 100 nt of target transcripts (Vasale et al., 2010). Roughly half of ERGO-1 class 26G RNAs map to coding loci, lincRNAs, or pseudogenes and half to loci that likely correspond to unannotated transcription units (Vasale et al., 2010). Many of their targets represent recently duplicated, non-conserved genes, suggesting ERGO-1 class 26G RNAs may buffer expression of newly acquired genes (Vasale et al., 2010; Fischer et al., 2011). A large-scale proteomics dataset (Schrimpf et al., 2009) shows peptides corresponding to less than 20\% of ERGO-1 class 26G RNA targets, but 54\% of all annotated coding genes, indicating that very few of these targets represent functional, coding loci (Fischer et al., 2011).

\section{Biogenesis of 26G RNAs: The ERI complex}

The ERI complex mediates 26G RNA biogenesis and is named for the enhanced (exogenous) RNAi (Eri) phenotype that is associated with compromise of ERGO-1 class 26G RNA function (Table 2 and Figure 1). Loss of ERI complex members also results in temperature-sensitive (ts) sterility at $25^{\circ} \mathrm{C}$ due to defective spermatogenesis as well as a high incidence of male progeny (Him) phenotype, indicative of increased X chromosome nondisjunction, due to compromised ALG-3/4 class 26G RNA function. The Eri and ts sterile phenotypes are explained in greater detail below. At the heart of the ERI complex is a core RdRP module consisting of RRF-3, DRH-3, and ERI-5. Associated with this module are DCR-1, accessory factors ERI-1b, ERI-3, RDE-4, and possibly the RNA phosphatase PIR-1. Analyses of DCR-1, ERI-1b, and ERI-5 complexes immuno-purified from gravid adult and embryo have revealed interactions with each other member of the ERI complex (Duchaine et al., 2006; Thivierge et al., 2012), and the $\sim 850 \mathrm{kD}$ estimated mass of the ERI complex is similar to the summed mass of these proteins, $\sim 810 \mathrm{kD}$ (Thivierge et al., 2012). The ERI complex shares only DCR-1 and RDE-4 with the RDE (exogenous $\underline{\mathrm{R} N A} \underline{\mathrm{i}}$ defective) complex, which mediates processing of dsRNA trigger to initiate the exo-RNAi pathway (Tabara et al., 2002). DCR-1 and ERI-1 also both interact with ERI-9, another factor implicated in ERGO-1 class 26G RNA biogenesis (Thivierge et al., 2012), suggesting that the ERI complex includes other transient interactors. 
Table 2. Protein factors acting in endogenous small RNA pathways.

\begin{tabular}{|c|c|c|c|}
\hline \multicolumn{2}{|l|}{ 26G RNA pathway factors } & \multirow{2}{*}{\begin{tabular}{|l|} 
Protein/domain(s) \\
RdRP
\end{tabular}} & \multirow[t]{2}{*}{ Orthologous Hs protein(s) } \\
\hline \multirow[t]{7}{*}{ ERI complex } & RRF-3 & & \\
\hline & DRH-3 & Dicer-related helicase & DDX58/IFIH1/DHX58 \\
\hline & ERI-5 & Tudor domain protein & \\
\hline & DCR-1/ERI-4 & Helicase, PAZ, RNase III & DICER1 \\
\hline & ERI-1b & $\begin{array}{l}\text { DEDDh-like 3' to 5' } \\
\text { exonuclease }\end{array}$ & ERI1 \\
\hline & ERI-3 & & \\
\hline & RDE-4 & dsRNA-binding domains & \\
\hline \multirow[t]{4}{*}{ Accumulation regulators } & ERI-9 & Putative RNA transferase & \\
\hline & ERI-6/7 & Superfamily I helicase & $\begin{array}{l}\text { IGHMBP2, MOV10 } \\
\text { (ERI-7) }\end{array}$ \\
\hline & MUT-16 & $\begin{array}{l}\text { P-rich domain, Q/N-rich } \\
\text { domains }\end{array}$ & \\
\hline & HENN-1 & Methyltransferase & HENMT \\
\hline \multirow[t]{2}{*}{ Argonautes } & ALG-3/4 & Argonaute & \\
\hline & ERGO-1 & Argonaute & \\
\hline \multicolumn{2}{|l|}{ 22G RNA pathway factors } & Protein/domain(s) & Orthologous Hs protein(s) \\
\hline \multirow[t]{4}{*}{ RdRP module } & RRF-1 & RdRP & \\
\hline & EGO-1 & $\operatorname{RdRp}$ & \\
\hline & DRH-3 & Dicer-related helicase & DDX58/IFIH1/DHX58 \\
\hline & EKL-1 & Tudor domain protein & \\
\hline \multirow{6}{*}{$\begin{array}{l}\text { WAGO 22G RNA } \\
\text { accumulation regulators: } \\
\text { Mutator foci }\end{array}$} & MUT-16 & $\begin{array}{l}\begin{array}{l}\text { P-rich domain, Q/N-rich } \\
\text { domains }\end{array} \\
\end{array}$ & \\
\hline & MUT-7 & 3' to 5' exonuclease & EXD3 \\
\hline & MUT-8/RDE-2 & & \\
\hline & MUT-2/RDE-3 & Beta-nucleotidyltransferase & TUT1/MTPAP \\
\hline & MUT-15 & & \\
\hline & MUT-14 & DEAD box RNA helicase & DDX4 \\
\hline \multirow{5}{*}{$\begin{array}{l}\text { WAGO 22G RNA } \\
\text { accumulation regulators: } \\
\text { RDE-10/RDE-11 complex }\end{array}$} & RDE-10 & Maelstrom domain & \\
\hline & RDE-11 & $\begin{array}{l}\text { RING-type zinc finger } \\
\text { domain }\end{array}$ & \\
\hline & RSD-2 & & \\
\hline & RSD-6 & Tudor domain protein & TDRD5 \\
\hline & HAF-6 & $\mathrm{ABC}$ transporter & ABCB8 \\
\hline
\end{tabular}




\begin{tabular}{|c|c|c|c|}
\hline \multicolumn{2}{|c|}{ 22G RNA pathway factors } & \multirow{2}{*}{\begin{tabular}{|l|} 
Protein/domain(s) \\
WAGO Argonaute
\end{tabular}} & \multirow[t]{2}{*}{ Orthologous Hs protein(s) } \\
\hline \multirow{8}{*}{$\begin{array}{l}\text { Cytoplasmic WAGO } \\
\text { Argonautes }\end{array}$} & WAGO-1 & & \\
\hline & WAGO-2 & WAGO Argonaute & \\
\hline & PPW-2/WAGO-3 & WAGO Argonaute & \\
\hline & WAGO-4 & WAGO Argonaute & \\
\hline & WAGO-5 & WAGO Argonaute & \\
\hline & WAGO-6 & WAGO Argonaute & \\
\hline & PPW-1/WAGO-7 & WAGO Argonaute & \\
\hline & WAGO-8 & WAGO Argonaute & \\
\hline \multirow{4}{*}{$\begin{array}{l}\text { Nuclear WAGO } \\
\text { Argonautes }\end{array}$} & HRDE-1/WAGO-9 & WAGO Argonaute & \\
\hline & WAGO-10 & WAGO Argonaute & \\
\hline & WAGO-11 & WAGO Argonaute & \\
\hline & NRDE-3/WAGO-12 & WAGO Argonaute & \\
\hline \multirow[t]{3}{*}{$\begin{array}{l}\text { Core nuclear RNAi } \\
\text { machinery }\end{array}$} & NRDE-2 & $\begin{array}{l}\text { Ser/Arg-rich domain, } \\
\text { HAT-like domain, } \\
\text { DUF1740 }\end{array}$ & NRDE2 \\
\hline & NRDE-1 & & \\
\hline & NRDE-4 & & \\
\hline \multirow{5}{*}{$\begin{array}{l}\text { Chromatin-level nuclear } \\
\text { RNAi effectors }\end{array}$} & HPL-2 & Heterochromatin protein & CBX1/CBX3 \\
\hline & MES-3 & Polycomb complex protein & \\
\hline & MES-4 & Trithorax protein & several \\
\hline & $\begin{array}{l}\text { SET-32 } \\
\end{array}$ & SET domain & \\
\hline & SET-25 & $\begin{array}{l}\text { Putative H3K9 } \\
\text { methyltransferase }\end{array}$ & SETBP1 \\
\hline $\begin{array}{l}\text { CSR-1 22G RNA } \\
\text { Argonaute }\end{array}$ & CSR-1 & Argonaute & \\
\hline $\begin{array}{l}\text { CSR-1 22G RNA } \\
\text { accumulation regulator }\end{array}$ & CDE-1 & poly(U) polymerase & ZCCHC6/ZCCHC11 \\
\hline \multicolumn{2}{|c|}{ 21U RNA pathway factors } & Protein/domain(s) & Orthologous Hs protein(s) \\
\hline \multirow[t]{2}{*}{ Transcription factors } & FKH-3/4/5 & $\begin{array}{l}\text { Forkhead transcription } \\
\text { factor }\end{array}$ & \\
\hline & UNC-130 & $\begin{array}{l}\text { Forkhead transcription } \\
\text { factor }\end{array}$ & $\begin{array}{l}\text { FOXD2/FOXD3/FOXD4/ } \\
\text { FOXD4L1-L6/FOXE1/ } \\
\text { FOXE3 }\end{array}$ \\
\hline Argonaute & PRG-1 & Piwi Argonaute & PIWIL1-4 \\
\hline Accumulation regulator & HENN-1 & Methyltransferase & HENMT \\
\hline
\end{tabular}




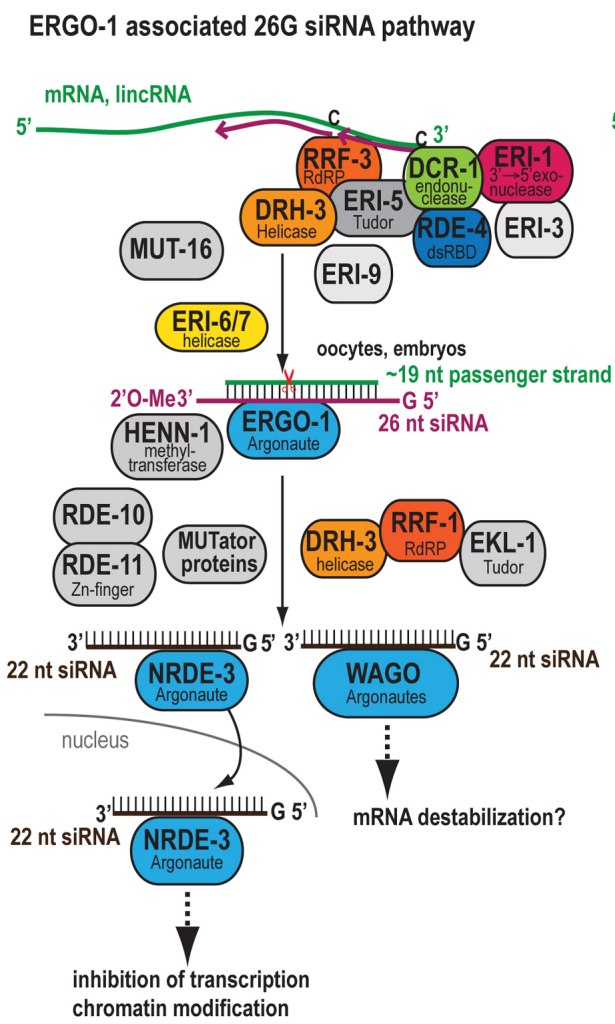

ALG-3/4 associated 26G siRNA pathway
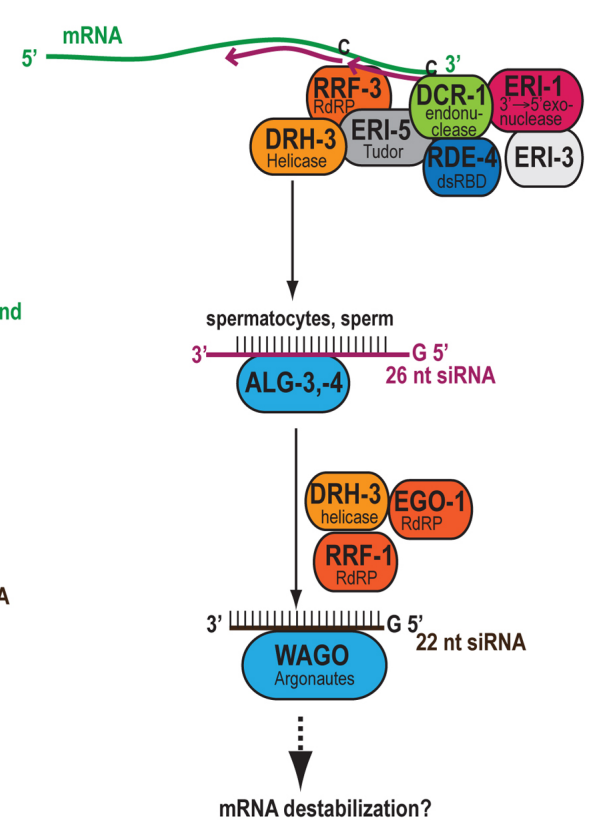

mRNA destabilization?

Figure 1. ERGO-1 and ALG-3/4 26G siRNA pathways. The templates for 26G siRNA generation are $\sim 500$ mRNAs and lincRNAs. A complex containing an RdRP and DCR-1 generates 26G siRNAs in both oocytes/embryos and spermatocytes/sperm. These 26G siRNAs associate with the Argonaute proteins ERGO-1 and ALG-3/4 respectively. 22G siRNA generation is triggered and requires another RdRP and multiple cofactors. These 22G siRNAs associate with WAGO class Argonautes to silence their targets in the soma through nuclear and cytoplasmic silencing pathways.

ERI complex factors show some interdependence and hierarchy of assembly. Most notably, ERI-5 is proposed to tether the RdRP module to DCR-1 to potentiate 26G RNA biogenesis. While the other factors are required for biogenesis of 26G RNAs, loss of ERI-5 merely attenuates their expression due to partial compensation by paralog EKL-1 (Thivierge et al., 2012). EKL-1 interacts with DRH-3 (Gu et al., 2009) and substitutes for ERI-5 in the RdRP module, but it does not interact with DCR-1 (Thivierge et al., 2012). Untethered to DCR-1, this EKL-1 RdRP module still produces 26G RNAs of normal genomic distribution and in a DCR-1-dependent manner, but 26G RNA accumulation is impaired (Thivierge et al., 2012).

\subsection{RRF-3}

RRF-3 is an RNA-dependent RNA polymerase (RdRP) (Smardon et al., 2000) required for 26G RNA biogenesis (Duchaine et al., 2006; Lee et al., 2006; Gent et al., 2009; Han et al., 2009; Pavelec et al., 2009; Conine et al., 2010; Gent et al., 2010; Vasale et al., 2010). C. elegans RdRPs and homologs catalyze primer-independent synthesis of antisense siRNAs (Makeyev and Bamford, 2002; Aoki et al., 2007; Pak and Fire, 2007; Sijen et al., 2007), with a preference for initiating transcription with GTP (Aoki et al., 2007) (see Section 8.1). The RdRP activity of RRF-3 is necessary for 26G RNA accumulation and function (Pavelec et al., 2009), suggesting that RRF-3 directly transcribes $26 \mathrm{G}$ RNAs. Each $26 \mathrm{G}$ RNA is likely generated as an independent transcript, as homologous RdRPs exhibit very low processivity (Makeyev and Bamford, 2002; Aoki et al., 2007), and 26G RNAs show irregular phasing and occasional overlap (Ruby et al., 2006; Vasale et al., 2010; Fischer et al., 2011). The N terminus of RRF-3 is divergent and may confer target specificity to the ERI RdRP module (Thivierge et al., 2012), as DRH-3 is also involved in biogenesis of other endo-siRNAs. In addition to loss of 26G RNAs, rrf-3 mutants show depletion of dependent secondary siRNAs, called WAGO 22G RNAs, whose biogenesis is triggered by 26G RNA targeting (Duchaine et al., 2006; Lee et al., 2006; Gent et al., 2009; Conine et al., 2010; Gent et al., 2010; Vasale et al., 2010). Loss of RRF-3 results in the characteristic sperm-origin ts sterile, Him, and Eri phenotypes associated with compromise of the 26G RNA pathway (Sijen et al., 2001; Simmer et al., 2002; Duchaine et al., 2006; Pavelec et al., 2009). 


\subsection{DRH-3}

The conserved Dicer-related helicase genes include two paralogs, $d r h-1$ and $d r h-3$, and a probable pseudogene, $d r h-2$. These genes are named for the similarity of their encoded DExD/H box helicase domains to that of DCR-1 and likely act as helicases on dsRNA intermediates during biogenesis of siRNAs (Tabara et al., 2002). The functions of DRH-1 and DRH-3 in siRNA biogenesis complexes differ. DRH-1 interacts with DCR-1, RDE-4, and Argonaute RDE-1 within the RDE complex to process primary exo-siRNAs from dsRNA trigger (Tabara et al., 2002; Thivierge et al., 2012). DRH-1 interacts directly with DCR-1 but does not participate in an RdRP module, whereas DRH-3 does not interact directly with DCR-1 but appears to represent an essential component of all $C$. elegans RdRP modules (Gu et al., 2009; Claycomb et al., 2009; Duchaine et al., 2006; Aoki et al., 2007; Nakamura et al., 2007). Thus, loss of DRH-3 results in loss of 26G RNAs (Vasale et al., 2010), although many other populations of small RNAs are also lost. Accordingly, the phenotypes associated with loss of DRH-3 are pleiotropic, with more severe phenotypes masking defects attributable to loss of 26G RNAs, and are discussed below with the relevant WAGO and CSR-1 22G RNA pathways.

\subsection{ERI-5}

ERI-5 is a protein containing two Tudor domains that putatively tethers the ERI complex RdRP module to DCR-1 (Duchaine et al., 2006; Thivierge et al., 2012). In vitro, recombinant ERI-5 binds recombinant DCR-1 but fails to bind recombinant RRF-3, suggesting that RRF-3 may be modified in vivo to generate Tudor domain-binding sites (Thivierge et al., 2012). Loss of ERI-5 results in the sperm-origin ts sterility and Eri phenotype of ERI complex mutants (Duchaine et al., 2006). However, due to redundancy with EKL-1, 26G RNAs are only depleted two-fold and the dependent (or secondary) WAGO 22G RNAs are depleted nine-fold in eri-5 mutant embryos, resulting in a two-fold up-regulation of 26G RNA target mRNAs (Duchaine et al., 2006; Thivierge et al., 2012).

\subsection{DCR-1}

Dicer contains a helicase domain, a PAZ domain, and dual RNase III motifs and is a conserved member of a family of RNase III nucleases that cleave dsRNA (Bernstein et al., 2001). dcr-1 encodes the sole Dicer homolog of C. elegans and acts in microRNA, primary exo-siRNA, and primary endo-siRNA biogenesis (Grishok et al., 2001; Ketting et al., 2001; Knight and Bass, 2001; Han et al., 2009). Loss of DCR-1 results in sterility, with abnormal oocyte formation and absence of fertilization (Grishok et al., 2001; Ketting et al., 2001; Knight and Bass, 2001), as well as heterochronic phenotypes due to defects in microRNA processing (Grishok et al., 2001; Ketting et al., 2001). Maternal inheritance of DCR-1 somewhat ameliorates somatic $d c r-1$ null mutant phenotypes. The $d c r-1$ mutant germline, however, shows an exogenous $\underline{R} N A i$-defective (Rde) phenotype and impaired transgene silencing, a related phenotype that reports on integrity of exo- and endo-RNAi pathways (Grishok et al., 2001; Ketting et al., 2001; Knight and Bass, 2001). In C. elegans extracts, DCR-1 dices long dsRNA into 23 bp duplexes (Ketting et al., 2001). Immuno-purified DCR-1 complexes digest dsRNA processively into siRNAs in the presence of ATP but terminate processing when ssRNA is encountered, demonstrating the specificity for dsRNA substrate (Ketting et al., 2001). Although 26G RNAs are transcribed by RRF-3, they do not show the signature 5' triphosphate of unprimed synthesis by an RdRP (Makeyev and Bamford, 2002). Rather, 26G RNAs show a 5' monophosphate characteristic of Dicer products, and indeed DCR-1 is required for their biogenesis (Han et al., 2009), as well as accumulation of dependent WAGO 22G RNAs (Duchaine et al., 2006; Lee et al., 2006). However, dsRNA substrates in embryo extract are cleaved with similar efficiency by DCR-1 regardless of the 5' nt identity, indicating that the 5' guanosine bias of 26G RNAs and other endo-siRNAs is not imposed by preferential DCR-1 processing (Welker et al., 2011).

\subsection{DCR-1 helicase domain}

The helicase domain of DCR-1 appears to play a specific role in biogenesis of primary siRNAs. The dcr-1(mg375) allele encodes a missense mutation in the helicase domain that does not affect ERI complex formation but abrogates biogenesis of 26G RNAs and dependent 22G RNAs (Pavelec et al., 2009, Welker et al., 2010). The dcr-1(mg375) helicase mutant shows sperm-origin ts sterile, Him, and Eri phenotypes, but is viable, fertile, and does not show heterochronic defects, indicating that DCR-1 helicase activity is dispensable for microRNA production (Pavelec et al., 2009, Welker et al., 2010). The RNAi sensitivity of the $d c r-1(m g 375)$ helicase mutant is less enhanced than that of other ERI complex mutants (Pavelec et al., 2009), suggesting that the helicase activity of DCR-1 may enhance primary exo-siRNA production from dsRNA triggers. This would be consistent with the theory that the helicase domain also functions as a translocase, allowing DCR-1 to catalyze multiple, processive cleavage events before dissociation from a dsRNA substrate (Welker et al., 2010). Study of the function of DCR-1 in cell-free 
embryo extracts suggests that the DCR-1 helicase domain facilitates cleavage of dsRNAs with blunt or 5 '-overhanging ends and promotes processive cleavage along the substrate. 3 '-overhanging termini are cleaved to 21-23 nt products without engaging the helicase domain, explaining the intact microRNA levels in the $d c r-1(m g 375)$ helicase mutant (Welker et al., 2011). The C. elegans Dicer homolog appears to measure from the 3' terminus of dsRNA substrate. Cleavage of short ( 40 bp) blunt-ended dsRNA yields a 26 nt small RNA with a 22-23 nt passenger strand. Processing of longer ( $\sim 100 \mathrm{bp})$ blunt-ended dsRNA in the presence of ATP yields both 26 and 27 nt species, with subsequent internal cleavages yielding $~ 23 \mathrm{nt}$ duplexes with 3 ' overhangs. Failure to produce $26 \mathrm{nt}$ species from internal cleavages strongly supports independent processing of 26G RNAs from short RdRP products rather than sequential cleavage of a long dsRNA precursor. Consistent with these in vitro data, deep sequencing libraries show evidence of shorter passenger strands antisense to 26G RNAs. Most commonly, the 5' nt of the sense read maps to nt 23 of the 26G RNA, corresponding to a 3 nt 3' overhang for the 26G RNA (Ruby et al., 2006; Fischer et al., 2011; Warf et al., 2012). While these sense fragments may be temporarily stabilized in the dsRNA duplex, the 3' ends of sense reads are highly variable, suggesting 3' to 5' degradation (Fischer et al., 2011; Warf et al., 2012).

\subsection{ERI-1b}

eri-1 encodes two isoforms of a conserved RNase that contains a DEDDh-like 3' to 5' exonuclease and a SAP/SAF-box domain (Kennedy et al., 2004). In vitro, ERI-1 partially degrades siRNAs with 2-nt 3' overhangs, but not ssRNA or siRNA internally hybridized to a long RNA (Kennedy et al., 2004). In vivo, ERI-1 is required for 26G RNA biogenesis and degrades the 3' end of the 5.8S rRNA, which pairs with the 5' end of the 25-28S rRNA. Loss of ERI-1 results in sperm-origin ts sterile, Him, and Eri phenotypes with loss of 26G RNAs and dependent 22G RNAs (Kennedy et al., 2004; Duchaine et al., 2006; Gabel and Ruvkun, 2008; Han et al., 2009; Conine et al., 2010; Gent et al., 2010). The significance of 5.8S rRNA processing is unknown but conserved in mammals. Both ERI-1 isoforms are cytoplasmically localized (Kennedy et al., 2004; Gabel and Ruvkun, 2008). Whereas either ERI-1 protein isoform is capable of rescuing 5.8S rRNA processing in vivo, eri-1 mutant phenotypes and 26G RNA accumulation are only rescued by expression of ERI-1b (Kennedy et al., 2004; Gabel and Ruvkun, 2008). ERI-1a may be insufficient for rescue because only ERI-1b interacts with DCR-1 (Duchaine et al., 2006), possibly via its extended C-terminal domain (Kennedy et al., 2004; Gabel and Ruvkun, 2008). ERI-1 exonuclease activity is required for 26G RNA accumulation and rRNA processing (Gabel and Ruvkun, 2008), but the precise function of ERI-1b in 26G RNA biogenesis is unclear. Possibly, ERI-1b recognizes 3' stem-loop structures in mRNA targets and removes excess nucleotides to generate a suitable RNA substrate for RRF-3-dependent synthesis (Duchaine et al., 2006). Alternatively, RRF-3 may synthesize dsRNA with short 3' overhangs that must be processed by ERI-1b to create blunt termini that engage DCR-1-mediated production of $26 \mathrm{nt}$ species (Welker et al., 2011).

\subsection{ERI-3}

ERI-3 is a protein without identifiable domains (Duchaine et al., 2006). eri-3 is encoded in an operon with taf-6.1 and can be expressed as a single polypeptide or as a fusion protein with TAF-6.1, which is also detected in immuno-purified DCR-1 complexes. Loss of eri-3 results in the characteristic phenotypes of ERI complex compromise, and ERI-3 may serve only to recruit ERI-1b to DCR-1 (Duchaine et al., 2006).

\subsection{RDE-4}

RDE-4 contains two dsRNA-binding motifs and binds long dsRNA preferentially in vitro without specificity for sequence or overhang structure (Tabara et al., 2002; Parker et al., 2006). RDE-4 protein mediates exo-RNAi in both germline and soma by interacting with DCR-1, DRH-1, and the Argonaute RDE-1 (Tabara et al., 1999). In vitro and in vivo studies of recombinant RDE-4 have dissected the contributions of the constituent domains to RDE-4 function: The C-terminal domain of RDE-4 directs its dimerization in solution (Parker et al., 2006). The 3' dsRNA-binding motif is important for dsRNA binding and mediates interaction with DCR-1 along with the linker between the motifs (Parker et al., 2008; Blanchard et al., 2011). RDE-4 binds dsRNA cooperatively (Parker et al., 2006; Parker et al., 2008) and promotes its accumulation in vivo (Tabara et al., 2002). During exo-RNAi, RDE-4 binds long trigger dsRNA in vivo, but not mRNA or amplified siRNAs (Tabara et al., 2002). RDE-4 is required for primary exo-siRNA and dependent 22G RNA production, but its absence can be partially bypassed by injection of prepared 24-25 nt siRNA duplexes (Parrish and Fire, 2001; Tabara et al., 2002). RDE-4 is required for full production of ERGO-1 class 26G RNAs and dependent 22G RNAs (Duchaine et al., 2006; Lee et al., 2006; Vasale et al., 2010; Welker et al., 2010). Although its role in ALG-3/4 class 26G RNA production has not been explicitly tested, RDE-4 appears to be required for detection of an endo-siRNA corresponding to ALG-3/4 class 26G target mRNA ssp-16 (Welker et al., 2010), and microarray profiling indicates that mRNA levels of ssp-16 are elevated in 
rde-4 mutant adult (Welker et al., 2007). In spite of this, loss of RDE-4 does not appear to result in the Him phenotype and highly penetrant sperm-origin sterility associated with compromise of ALG-3/4 class 26G RNAs (Welker et al., 2010; Blanchard et al., 2011). Perhaps loss of RDE-4 does not fully abrogate 26G RNA biogenesis, as target desilencing is less profound in rde-4 than eri-1 mutant embryo (Zhang et al., 2011). The function of RDE-4 in 26G RNA biogenesis remains somewhat unclear. The preference shown by RDE-4 for binding of long dsRNA may promote exo-RNAi by aiding release of dsRNA siRNA duplexes after DCR-1 processing (Parker et al., 2006), but argues against a role for RDE-4 in binding and stabilizing the likely very short dsRNA 26G RNA precursor.

\section{Other factors implicated in 26G RNA accumulation}

\subsection{ERI-9}

ERI-9 is a worm-specific putative RNA transferase (Pavelec et al., 2009; Zhuang and Hunter, 2012) that interacts with DCR-1 (Duchaine et al., 2006) and is required only for ERGO-1 class 26G RNA and dependent 22G RNA accumulation (Pavelec et al., 2009). Loss of ERI-9 results in the Eri phenotype associated with loss of ERGO-1 class 26G RNAs, but neither the Him phenotype nor the sperm-origin ts sterility associated with loss of ALG-3/4 class 26G RNAs (Pavelec et al., 2009).

\subsection{ERI-6/7}

ERI-6/7 is a helicase protein required only for ERGO-1 class 26G RNA and dependent 22G RNA accumulation (Fischer et al., 2008; Fischer et al., 2011). The ERI-6/7 protein is encoded by antiparallel eri-6 and eri-7 pre-mRNAs that are trans-spliced to generate a fusion mRNA; these two genes constitute a single, contiguous gene in $C$. briggsae and the $C$. elegans CB4856 isolate (Fischer et al., 2008). Although eri-7 is classified as oogenesis-enriched (Reinke et al., 2004), transcriptional fusion reporters indicate that these genes are also somatically expressed (Fischer et al., 2008). ERI-6/7 is predominantly cytoplasmically localized, suggesting RNA helicase function (Fischer et al., 2008). Loss of ERI-6/7 results in an Eri phenotype (Fischer et al., 2008), but only moderately decreased fertility (Fischer et al., 2011). ALG-3/4 class 26G RNAs and all other major classes of $C$. elegans small RNAs are not decreased by loss of ERI-6/7 (Fischer et al., 2011). Interaction between ERI-6/7 and the ERI complex has not been detected, suggesting that ERI-6/7 may function at a later step in ERGO-1 class 26G RNA accumulation, such as Argonaute loading.

\subsection{MUT-16}

MUT-16 is a worm-specific protein with proline-rich and glutamine/asparagine-rich regions (Vastenhouw et al., 2003) that functions critically in Mutator foci in the WAGO 22G RNA pathway (Phillips et al., 2012) and will therefore be further discussed below (Section 10). Loss of MUT-16 results in severe depletion of ERGO-1 class $26 \mathrm{G}$ RNAs and dependent 22G RNAs, but not ALG-3/4 class 26G RNAs (Zhang et al., 2011). Some C. elegans laboratory strains, such as the $d c r-1(m g 375)$ helicase mutant, contain a mut-16(mg461) mutation; this allele fails to express somatic MUT-16 fully, potentially compromising somatic 26G RNA production and complicating interpretation of experimental results pertaining to the ERGO-1 class 26G RNA pathway or somatic WAGO pathway-dependent gene silencing (Zhang et al., 2011).

\subsection{Other Mutator (MUT) proteins}

ERGO-1 class 26G RNAs also require MUT-2, MUT-7, and MUT-15 and show minor dependence on MUT-8 and MUT-14 (Zhang et al., 2011). These proteins are recruited by MUT-16 to form Mutator foci in the germline for WAGO 22G RNA amplification and target silencing (see Section 10) (Phillips et al., 2012). ALG-3/4 class 26G RNAs are modestly decreased by loss of MUT-7, but appear intact upon loss of the other Mutator foci MUT proteins (Zhang et al., 2011). The dependence of ERGO-1 class 26G RNAs on this set of factors suggests that Mutator foci may also serve as amplification centers for primary endo-siRNA biogenesis.

\section{26G RNA Argonaute loading}

The ERGO-1 and ALG-3/4 Argonautes are required for accumulation of their respective 26G RNA populations (Han et al., 2009; Conine et al., 2010; Gent et al., 2010; Vasale et al., 2010), but they are not found in the ERI complex and are thought to act downstream of biogenesis. While many of the Argonautes encoded by $C$. elegans do not show conservation of the three critical catalytic residues mediating slicer activity, all of the Argonautes that bind primary small RNAs (ERGO-1, ALG-3/4, PRG-1/2, RDE-1, ALG-1/2, and, putatively, 
CSR-1) show intact catalytic triads in their RNase H-related PIWI domains (Yigit et al., 2006). Although the slicer activity of an Argonaute refers generally to the ability to catalyze target cleavage, it appears also to play a critical role in effector complex maturation through passenger strand removal. The catalytic activity of RDE-1, the Argonaute that binds primary exo-siRNAs, is required only for efficient removal of the passenger strain; thus, the catalytic mutant of RDE-1 shows only a partial Rde phenotype attributable to impaired target mRNA interaction (Steiner et al., 2009). A putative ERGO-1 catalytic mutant exhibits an Eri phenotype (Fischer et al., 2011). This indicates that catalytic activity is required for ERGO-1-mediated triggering of WAGO 22G RNAs, but 26G RNA and passenger strand levels have not been assessed. The ergo-1 mutant shows vastly depleted ERGO-1 class 26G RNA levels but two-fold increased passenger strand levels (Fischer et al., 2011), supporting a role for ERGO-1 slicer activity in liberating the passenger strand from a 26G RNA dsRNA intermediate. In comparison, both mature ERGO-1 class 26G RNAs and passenger strands are depleted by loss of ERI-1 or ERI-6/7, suggesting function upstream of 26G RNA duplex formation (Fischer et al., 2011). Similar analyses of catalytically inactive ALG-3 or ALG-4 have not been reported.

\subsection{ALG-3/4}

The highly homologous Argonautes ALG-3 and ALG-4 redundantly bind and stabilize 26G RNAs generated in spermatogenic germline and mediate their effector functions (Han et al., 2009; Conine et al., 2010). alg-3 mRNA and protein are enriched in male worms and depleted in female worms (Han et al., 2009; Conine et al., 2010). In the spermatogenic germline, ALG-3 expression begins in postpachytene spermatocytes, showing cytoplasmic localization with enrichment in P granules (Conine et al., 2010). After spermatogenesis, ALG-3 is detected only in the spermatheca, where it is confined to residual bodies after mature, postmeiotic spermatids have budded off (Conine et al., 2010). The fertility of the alg-3 or alg-4 single mutants does not differ significantly from that of wild type (Han et al., 2009; Conine et al., 2010), reflecting their redundancy, but loss of both ALG-3 and ALG-4 impairs fertility at $20^{\circ} \mathrm{C}$ and results in the characteristic sperm-origin ts sterility at $25^{\circ} \mathrm{C}$ (Han et al., 2009; Conine et al., 2010). The RNAi sensitivity of the single and double mutants is wild type (Han et al., 2009).

\subsection{ERGO-1}

The Argonaute ERGO-1 binds and stabilizes 26G RNAs generated in oogenic germline, embryo, and likely beyond and mediates their effector function (Han et al., 2009; Pavelec et al., 2009; Gent et al., 2010; Vasale et al., 2010). ERGO-1 is nearly absent from L3 and L4 larva and young adult (Vasale et al., 2010), paralleling decreased detection of ERGO-1 class 26G RNAs during these stages (Han et al., 2009; Billi et al., 2012). In the hermaphrodite oogenic germline, ERGO-1 expression begins at pachytene exit and persists into embryo, showing cytoplasmic localization throughout (Billi et al., 2012). The ergo-1 mutant exhibits the characteristic Eri phenotype associated with loss of ERGO-1 class 26G RNAs, but only a minor fertility defect and no Him phenotype (Yigit et al., 2006; Han et al., 2009; Pavelec et al., 2009).

\subsection{HENN-1}

HENN-1 is the C. elegans ortholog of HEN1 methyltransferase (Park et al., 2002). HENN-1 catalyzes 2'-O-methylation of the 3' terminus of small RNAs associated with Argonautes of the PIWI clade (Billi et al., 2012; Montgomery et al., 2012), namely, ERGO-1 class 26G RNAs and 21U RNAs (Ruby et al., 2006; Billi et al., 2012; Kamminga et al., 2012; Montgomery et al., 2012). Direct interaction between HENN-1 and either ERGO-1 or PRG-1 has not been demonstrated (Billi et al., 2012; Kamminga et al., 2012), and recombinant HENN-1 is capable of methylating RNA oligomers in the presence of S-adenosyl methionine (Kamminga et al., 2012). Nevertheless, HENN-1 appears to methylate small RNAs only following Argonaute binding, as loss of ERGO-1 results in loss of methylation for the rare residual ERGO-1 class 26G RNAs (Billi et al., 2012). henn-1 mRNA and protein are detected at all stages in both germline and soma with strongest expression in germline and embryo (Billi et al., 2012; Kamminga et al., 2012). HENN-1 is detected throughout male and female germlines, with proximal oocytes showing cytoplasmic and intense nucleoplasmic signal; the nucleoplasmic enrichment is lost upon fertilization (Billi et al., 2012). During sperm maturation, HENN-1 becomes enriched in residual bodies, suggesting possible exclusion from mature spermatids (Billi et al., 2012). In embryos, HENN-1, like ERGO-1, is abundant and diffusely cytoplasmic (Billi et al., 2012; Kamminga et al., 2012). HENN-1-mediated methylation is critical for ERGO-1 class 26G RNA stability and inheritance into the embryo; in the absence of HENN-1, ERGO-1 class 26G RNAs show size heterogeneity (Billi et al., 2012; Kamminga et al., 2012) and increased levels of non-templated nucleotide additions (Montgomery et al., 2012). However, this trimming and tailing activity is not limited to unmethylated 26G RNAs. Analysis of trimming and tailing rates by small RNA class reveals that the methylated 21U RNAs show the lowest frequency and ALG-3/4 class 26G RNAs the highest, but ERGO-1 class 26G RNAs show a trimming and tailing 
rate nearly as high as that of ALG-3/4 class 26G RNAs (Montgomery et al., 2012). 21U RNAs show significantly decreased perdurance in the absence of HENN-1, but their initial accumulation is less severely affected than that of ERGO-1 class 26G RNAs (Billi et al., 2012; Kamminga et al., 2012; Montgomery et al., 2012). The relevance of HENN-1 to 21U RNA accumulation and stability are discussed further below. It is unclear how HENN-1 affects $22 \mathrm{G}$ RNA levels. Global levels of both WAGO and CSR-1 22G RNAs are decreased in the absence of HENN-1 by $\sim 30 \%$ without major changes in size or frequency of addition of non-templated nucleotides (Kamminga et al., 2012), contributing to decreased detection of $22 \mathrm{G}$ RNAs dependent upon methylated primary siRNAs (Billi et al., 2012; Montgomery et al., 2012). The effects on target mRNAs are difficult to interpret, as global mRNA analysis shows general downregulation of germline-expressed genes upon loss of HENN-1; however, ERGO-1 class 26G RNA target mRNAs are not downregulated, hinting at impaired target silencing (Kamminga et al., 2012), and another report identifies significant upregulation of several ERGO-1 class 26G RNA targets (Montgomery et al., 2012). It is possible that the general depletion of germline mRNAs causes decreased 22G RNA levels due to decreased template-dependent synthesis, but another explanation may be that mRNA levels of critical 22G RNA pathway factors such as PPW-2 and MUT-7 are decreased by loss of HENN-1 (Kamminga et al., 2012). This may also explain a curious phenotype of henn-1 mutants: while the soma shows an Eri phenotype, presumably due to somatic ERGO-1 class 26G RNA depletion, the germline is Rde (Billi et al., 2012; Kamminga et al., 2012). Loss of HENN-1 also results in slightly decreased fertility at $25^{\circ} \mathrm{C}$ and a mild Him phenotype (Billi et al., 2012; Kamminga et al., 2012).

\section{26G RNA effector function}

Loss of factors involved in ERGO-1 and/or ALG-3/4 class 26G RNA biogenesis or accumulation results in desilencing of the relevant population of complementary target mRNAs (Duchaine et al., 2006; Lee et al., 2006; Gent et al., 2009; Han et al., 2009; Pavelec et al., 2009; Conine et al., 2010; Gent et al., 2010; Vasale et al., 2010) (Table 3). Factors that play lesser roles in accumulation such as ERI-5 or Mutator foci MUT proteins show less pronounced silencing defects (Duchaine et al., 2006; Zhang et al., 2011). While several factors have been identified that appear to contribute exclusively to ERGO-1 class 26G RNA accumulation (Pavelec et al., 2009; Fischer et al., 2011; Billi et al., 2012; Kamminga et al., 2012; Montgomery et al., 2012; Zhuang and Hunter, 2011), ALG-3 and ALG-4 remain the only factors known to be specifically required for accumulation of ALG-3/4 class 26G RNAs (Han et al., 2009; Conine et al., 2010). 26G RNAs repress target mRNA expression in their cognate cell types; loss of $26 \mathrm{G}$ RNAs does not result in ectopic expression within other cell types of the native male or female germline or inappropriate target expression in the opposite germline (Han et al., 2009).

Table 3. Functions of endogenous small RNAs.

\begin{tabular}{|c|c|c|c|c|}
\hline Small RNA & Features of targets & $\begin{array}{l}\text { Targeting } \\
\text { Mechanism }\end{array}$ & $\begin{array}{l}\text { Cofactors involved } \\
\text { in function }\end{array}$ & Phenotypes of loss \\
\hline $\begin{array}{l}\text { ERGO-1 class } 26 \mathrm{G} \\
\text { RNA }\end{array}$ & $\begin{array}{l}\text { Duplicated genes, } \\
\text { lincRNA genes, } \\
\text { noncoding loci }\end{array}$ & $\begin{array}{l}\text { Silencing in cis and in } \\
\text { trans } \\
\text { (mismatch-tolerant) }\end{array}$ & $\begin{array}{l}\text { WAGO (NRDE-3) } \\
\text { 22G RNAs, } \\
\text { RDE-10/RDE-11, } \\
\text { RSD-2, RSD-6, } \\
\text { HAF-6 }\end{array}$ & Eri \\
\hline $\begin{array}{l}\text { ALG-3/4 class } 26 \mathrm{G} \\
\text { RNA }\end{array}$ & $\begin{array}{l}\text { Spermatogenesis- } \\
\text { enriched genes }\end{array}$ & Unknown & WAGO 22G RNAs & $\begin{array}{l}\text { ts Ste (male origin), } \\
\text { Him }\end{array}$ \\
\hline $\begin{array}{l}\text { NRDE-3-associated } \\
\text { 22G RNA }\end{array}$ & $\begin{array}{l}\text { Somatic targets of } \\
\text { exo-siRNAs and } \\
\text { ERGO-1 class } 26 \mathrm{G} \\
\text { RNAs }\end{array}$ & $\begin{array}{l}\text { Silencing in cis and in } \\
\text { trans } \\
\text { (mismatch-tolerant) }\end{array}$ & $\begin{array}{l}\text { NRDE-1, NRDE-2, } \\
\text { NRDE-4 }\end{array}$ & Nrde \\
\hline $\begin{array}{l}\text { HRDE-1-associated } \\
\text { 22G RNA }\end{array}$ & $\begin{array}{l}\text { Germline targets of } \\
\text { exo-siRNAs and } 21 \mathrm{U} \\
\text { RNAs }\end{array}$ & & $\begin{array}{l}\text { NRDE-1, NRDE-2, } \\
\text { NRDE-4, HPL-2, } \\
\text { MES-4, SET-32, } \\
\text { SET-25 }\end{array}$ & Nrde, Him, Mrt \\
\hline $\begin{array}{l}\text { Cytoplasmic } \\
\text { WAGO-associated } \\
\text { 22G RNA }\end{array}$ & $\begin{array}{l}\text { Targets of } 26 \mathrm{G} \\
\text { RNAs, } 21 \mathrm{U} \text { RNAs, } \\
\text { RDE-1-associated } \\
\text { siRNAs }\end{array}$ & & & Rde, Mut \\
\hline
\end{tabular}




\begin{tabular}{|l|l|l|l|l|}
\hline Small RNA & Features of targets & $\begin{array}{l}\text { Targeting } \\
\text { Mechanism }\end{array}$ & $\begin{array}{l}\text { Cofactors involved } \\
\text { in function }\end{array}$ & Phenotypes of loss \\
\hline CSR-1 22G RNA & $\begin{array}{l}\text { Germline-expressed } \\
\text { coding genes }\end{array}$ & Unknown & Emb \\
\hline 21U RNA (Type I) & $\begin{array}{l}\text { Transposon genes, } \\
\text { pseudogenes } \\
\text { (depleted of } \\
\text { protein-coding genes) }\end{array}$ & $\begin{array}{l}\text { Silencing in trans } \\
\text { (mismatch-tolerant) }\end{array}$ & $\begin{array}{l}\text { HRDE-1-bound 22G } \\
\text { RNAs, cytoplasmic } \\
\text { WAGO 22G RNAs }\end{array}$ & ts Ste, Him \\
\hline $\begin{array}{l}\text { TSS-associated 21U } \\
\text { RNA (Type II) }\end{array}$ & $\begin{array}{l}\text { Targets and function } \\
\text { unknown }\end{array}$ & $\begin{array}{l}\text { Silencing in trans } \\
\text { (seed sequence } \\
\text { pairing) }\end{array}$ & & microRNA-specific \\
\hline microRNA & Diverse & & & \\
\hline
\end{tabular}

During 26G RNA biogenesis, DCR-1 likely catalyzes the cleavage of the mRNA template in generating the dsRNA intermediate (Welker et al., 2011), thereby reducing mRNA levels. Subsequently, 26G RNA Argonaute recognition may also trigger target cleavage. However, neither is likely to represent a significant means of target silencing, as WAGO $22 \mathrm{G}$ RNAs triggered by $26 \mathrm{G}$ RNA targeting are the major effectors of the 26G RNA endo-RNAi pathway. These secondary siRNAs, discussed in depth below, are strictly required for 26G RNA target silencing (Conine et al., 2010; Gent et al., 2010; Vasale et al., 2010), as well as silencing of other small RNA pathway targets (Figure 2). It is not known precisely how 26G RNA RISC association triggers 22G formation, but target transcripts show 26G RNA-dependent accumulation of WAGO 22G RNAs (Conine et al., 2010; Gent et al., 2010; Vasale et al., 2010), and many 22G and 26G RNAs originate from the same 5' nt (Gent et al., 2010).

WAGO-associated $22 \mathrm{G}$ siRNA generation

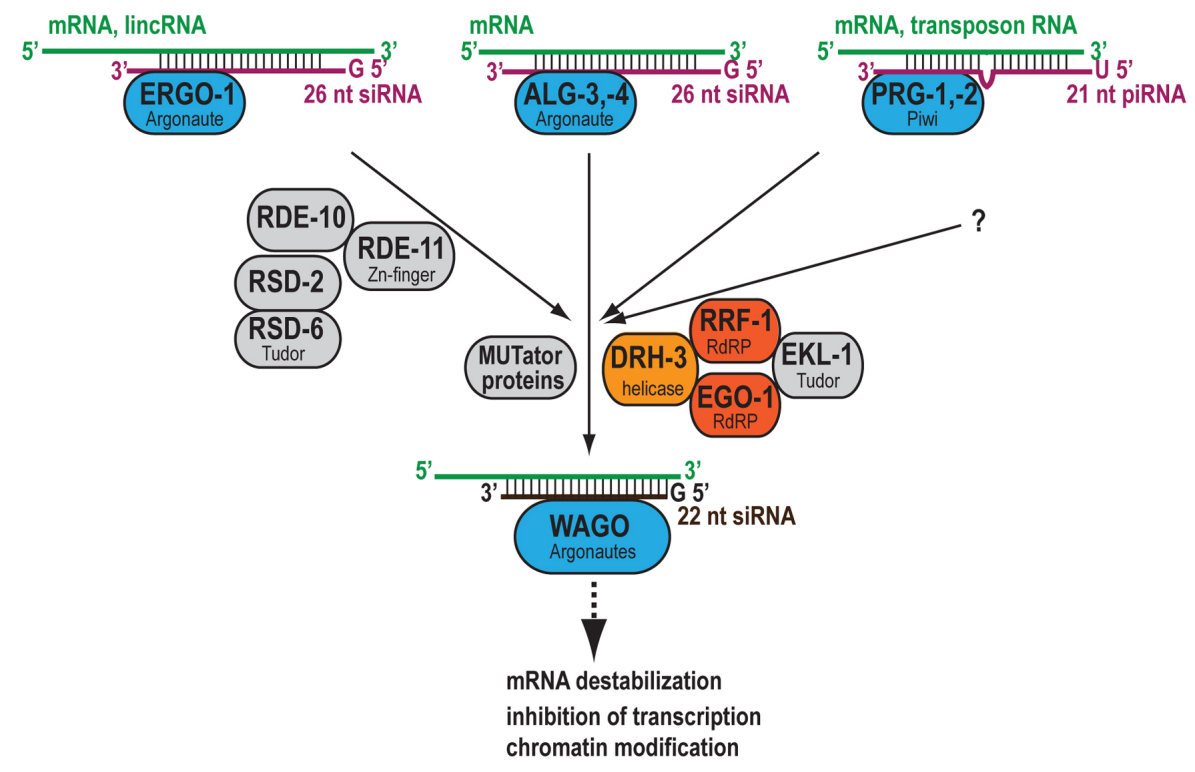

Figure 2. Different classes of primary small RNAs trigger secondary siRNA generation. 26G siRNAs and 21U/piRNAs trigger secondary 22G siRNA generation by the RdRPs RRF-1 and EGO-1, in conjunction with the helicase DRH-3 and the Tudor domain protein EKL-1. Six mutator proteins that form perinuclear Mutator foci are required for $22 \mathrm{G}$ siRNA production in the germline. The RDE-10/11 complex promotes secondary $22 \mathrm{G}$ siRNA amplification in the soma.

22G RNAs amplify the silencing signal of 26G RNAs and increase the perdurance of the repression. ERGO-1 class 26G RNA levels peak in embryos and decline significantly throughout larval development (Han et al., 2009; Gent et al., 2010; Vasale et al., 2010); however, ERGO-1 class 26G RNAs primarily target transcripts that are not germline-intrinsic (Reinke et al., 2004; Han et al., 2009), and triggering the production of WAGO 22G RNAs enables their silencing influence to persist through larval development (Han et al., 2009; Gent et al., 2010; Vasale et al., 2010). Similarly, 26G RNAs and ALG-3/4 are depleted in mature sperm, but WAGO 22G RNA Argonaute 
WAGO-1 is abundant in mature sperm (Conine et al., 2010). This precludes significant inheritance of paternal 26G RNAs, but evidently not dependent 22G RNAs: an ALG-3/4 class 26G RNA-dependent 22G RNA can be detected even at the L1 larval stage (Han et al., 2009).

Analysis of 26G RNA-dependent 22G RNAs further suggests that 26G RNAs target transcripts in trans as well as in cis. The X-cluster (Ambros et al., 2003) describes a region of the $\mathrm{X}$ chromosome from which abundant $22 \mathrm{Gs}$, but none or very few 26Gs, are generated (Vasale et al., 2010). Accumulation of X-cluster 22G RNAs requires ERGO-1 (Yigit et al., 2006) and a particular ERGO-1 class 26G RNA derived from the K02E2.11 transcript, which shares no other significant homology with the X-cluster (Montgomery et al., 2012). This particular 26G RNA shows multiple putative target sites throughout the X-cluster. None is perfectly complementary, and the best sites contain a minimum of three adjacent, central mismatches and two wobble pairs (Montgomery et al., 2012). This indicates that 26G RNA targeting of mRNA targets to initiate $22 \mathrm{G}$ RNA production in trans is mismatch-tolerant, suggesting immense targeting capacity.

\subsection{Sperm-origin ts sterility due to loss of ALG-3/4 class 26G RNAs}

As indicated above, compromise of ALG-3/4 class 26G RNA pathway activity results in sterility at $25^{\circ} \mathrm{C}$ due to defective spermatogenesis and spermiogenesis (Han et al., 2009; Pavelec et al., 2009; Conine et al., 2010). The Him phenotype also results from X chromosome missegregation during compromised spermatogenesis. eri-1, eri-3, and $r r f-3$ mutants do not show maternal rescue of this fertility defect, unlike the Eri phenotype (Zhuang and Hunter, 2011). 26G RNA and dependent $22 \mathrm{G}$ RNA production is lost in eri-1, eri-3, eri-5, and rrf-3 mutants at both elevated and permissive temperatures (Duchaine et al., 2006), indicating that it is not siRNA production that is sensitive to temperature but rather that these siRNAs are only required for functional spermatogenesis at elevated temperatures. The temperature-sensitive period for fertility in the absence of ALG-3/4 class 26G RNAs coincides with spermatogenesis at the L4 larval stage (Gent et al., 2009; Pavelec et al., 2009; Conine et al., 2010).

The defects occurring during spermatogenesis at $25^{\circ} \mathrm{C}$ in the absence of $26 \mathrm{G}$ RNAs have been detailed in $r r f-3$ and $d c r-1(m g 375)$ helicase mutants as well as in the alg-3; alg-4 double mutant, which lacks only ALG-3/4 class 26G RNAs (Gent et al., 2009; Pavelec et al., 2009; Conine et al., 2010). The phenotypes are highly similar, suggesting that compromise of only the male germline-expressed class of $26 \mathrm{G}$ RNAs is sufficient to cause these defects; consistent with this, loss of ERGO-1 ERI-9, or ERI-6/7 results on only very mild decreases in fertility at $25^{\circ} \mathrm{C}$ (Han et al., 2009; Fischer et al., 2011).

The spermatogenesis defects associated with compromised ALG-3/4 class 26G RNA target silencing are as follows: (1) mutant gonads show delayed spermatogenesis and decreased production of sperm-like nuclei; (2) nuclear abnormalities first become evident in primary spermatocytes; (3) some late-stage spermatocytes show chromatin bridges and arrest as multinucleate masses or produce spermatids with large, misshapen, or multiple nuclei; (4) spermatocytes that develop without obvious morphological abnormalities produce smaller numbers of residual bodies and sperm; (5) mutant sperm show abnormal wreaths of microtubules surrounding nuclei; and (6) activation of these mutant sperm is impaired - many fail to form pseudopods and instead show long spike structures and impaired motility (Gent et al., 2009; Pavelec et al., 2009; Conine et al., 2010).

Precisely how ALG-3/4 class 26G RNAs promote thermotolerant fertility is unknown. It is unlikely that desilencing of a single target results in these sperm development and motility defects, as individual depletion of 68 spermatogenesis-enriched transcripts desilenced in eri- 1 or $r r f-3$ mutants failed to suppress the ts sterility and EMS mutagenesis failed to identify any suppressors among several million genomes screened (Pavelec et al., 2009). Many of these defects are observed with variable penetrance at $20^{\circ} \mathrm{C}$, but nearly all sperm show the abnormal spike structures during activation at $25^{\circ} \mathrm{C}$, correlating with the penetrant ts sterility. Many ALG-3/4 class 26G RNAs target transcripts encoding major sperm proteins (Asikainen et al., 2007; Han et al., 2009; Pavelec et al., 2009; Conine et al., 2010), which assemble into filamentous fibers in the pseudopod. One function of 26G RNAs may be to promote male fertility by limiting excess accumulation of major sperm proteins. Additionally, $r r f-3$ and eri-1 mutant hermaphrodites cultured at $23^{\circ} \mathrm{C}$ generate embryos with significant spindle structure abnormalities including tripolar spindles or male pronuclei with supernumerary microtubule asters (Gent et al., 2009). These defects, as well as the abnormal microtubule wreaths in spermatids (Gent et al., 2009) and the chromosome segregation defects in spermatocytes (Gent et al., 2009; Pavelec et al., 2009; Conine et al., 2010), suggest a role for ALG-3/4 class 26G RNAs in regulating microtubule organization during spermatogenesis. Alternatively, these microtubule defects may reflect abnormalities in chromosome complements resulting from dysfunctional spermatogenesis. 


\subsection{Enhanced RNAi sensitivity due to loss of ERGO-1 class 26G RNAs}

In C. elegans, exo-RNAi and endo-RNAi engage a common downstream pathway to effect target silencing: the WAGO 22G RNAs. The earliest evidence for intersection between these two pathways was the identification of mutants that show both Rde and Mutator (Mut, loss of transposon silencing) phenotypes (Ketting et al., 1999; Tabara et al., 1999), indicating that common mechanisms mediate exo-RNAi and germline transposon silencing. Discovery of distinct primary and secondary phases of exo-RNAi first prompted the hypothesis that loss of RRF-3 might release limiting, common cofactors for function in the amplification phase of RNAi (Sijen et al., 2001). The WAGO Argonautes appear to represent these limiting factors in RNAi: loss of one can impair sensitivity to exogenous RNAi (Tijsterman et al., 2002b; Yigit et al., 2006), and overexpression of one enhances accumulation of 22G RNAs and RNAi sensitivity (Yigit et al., 2006). Accordingly, loss of ERGO-1 class 26G RNAs results in an Eri phenotype due to decreased competition for WAGO Argonaute occupancy by secondary siRNAs (Duchaine et al., 2006; Lee et al., 2006; Yigit et al., 2006; Gu et al., 2009), in line with an earlier observation that loss of ERI-1 increases accumulation of exo-RNAi-triggered siRNAs (Kennedy et al., 2004). The converse is also true: in the absence of exogenous dsRNA, RDE-1 scavenges diverse small RNAs and triggers endogenous somatic 22G RNA production (Welker et al., 2007; Gu et al., 2009; Corrêa et al., 2010); loss of RDE-1 and these dependent endo-siRNAs enhances accumulation of an ERGO-1-dependent somatic 22G RNA (Yigit et al., 2006).

Loss of ERGO-1 class 26G RNAs, but not ALG-3/4 class 26G RNAs, results in the Eri phenotype of ERI complex mutants (Han et al., 2009). Only ERGO-1 class 26G RNAs are inherited by offspring to generate abundant 22G RNAs and effect target silencing (Conine et al., 2010; Gent et al., 2010; Vasale et al., 2010). Eri mutants show strong maternal, but not paternal, rescue of the Eri phenotype in the soma (Zhuang and Hunter, 2011), consistent with this phenotype arising due to liberation of WAGO Argonautes in the absence of maternally inherited ERGO-1 class 26G RNAs and dependent 22G RNAs. It is possible that upregulation of target mRNAs encoding factors such as helicases and dsRNA-binding proteins may also contribute to the Eri phenotype associated with loss of ERGO-1 class 26G RNAs (Fischer et al., 2011).

\section{22G RNAs comprise two major small RNA pathways}

Early capture of $C$. elegans small RNAs using a protocol insensitive to 5' structure indicated that a majority of antisense small RNAs are $22 \mathrm{nt}$ in length (Ambros et al., 2003). Protocols selective for 5' monophosphorylated species failed to recover these sequences effectively (Ruby et al., 2006; Lim et al., 2003), revealing a 5' structure different from that of the 26G RNAs. Subsets of these small RNAs were subsequently shown to exhibit a 5' triphosphate (Pak and Fire, 2007) and a 3' hydroxyl (Pak and Fire, 2007; Sijen et al., 2007; Gu et al., 2009). Finally, 22 nt siRNAs in C. elegans show a prominent 5' guanosine bias (Ambros et al., 2003; Ruby et al., 2006; Gu et al., 2009), distinguishing these as $22 \mathrm{G}$ RNAs.

As a whole, 22G RNAs target about $~ 50 \%$ of the annotated coding genome, with most $22 \mathrm{G}$ RNAs targeting unique genome sequences (Gu et al., 2009). 22G RNAs mapping antisense to mRNAs show enrichment primarily at transcript 3' ends, consistent with RdRP engagement at the mRNA 3' terminus, but some also show enrichment at 5' ends (Gu et al., 2009). Most 22G RNAs are germline expressed and deposited into embryo, potentially coinherited with their mRNA targets (Gu et al., 2009). The 22G RNAs comprise two distinct classes of small RNAs that are synthesized by similar RdRP modules but engage unique pathways mediated by specific Argonautes and cofactor proteins to effect entirely distinct outcomes. The WAGO 22G RNAs bind semi-redundant Argonautes of the worm-specific WAGO clade to mediate silencing of certain protein-coding genes, transposons, pseudogenes, and cryptic loci through both transcriptional and post-transcriptional mechanisms (Guang et al., 2008; Gu et al., 2009; Burkhart et al., 2011; Burton et al., 2011; Ashe et al., 2012; Buckley et al., 2012; Gu, S.G., et al. 2012; Luteijn et al., 2012; Shirayama et al., 2012). The CSR-1 22G RNAs bind CSR-1, another WAGO Argonaute, and target germline-expressed genes to fulfill an essential role in promoting chromosome segregation (Claycomb et al., 2009). Thus, 22G RNAs target both silent and expressed loci genome-wide to maintain the germline.

\section{22G RNA biogenesis: The 22G RNA RdRP module}

RdRPs RRF-1 and EGO-1 both contribute to biogenesis of 22G RNAs (Figure 2). The ego-1; rrf-1 double mutant lacks 22G RNAs synthesized de novo in the germline (Gu et al., 2009). EGO-1 and RRF-1 proteins show greater than 50\% sequence identity (Smardon et al., 2000) and collaborate to generate WAGO 22G RNAs, but EGO-1 alone is required for CSR-1 22G RNAs (Claycomb et al., 2009; Gu et al., 2009). Accordingly, the ego-1 transcript is classified as germline-intrinsic (Reinke et al., 2004), and ego-1 mRNA and protein are enriched in 
young adult, adult, and embryo but nearly absent during larval development (Smardon et al., 2000; Vought et al., 2005; Claycomb et al., 2009). EGO-1 protein is also enriched in the nuclear fraction of cell lysates (Maine et al., 2005). In contrast, a transgene expressing GFP::RRF-1 that rescues the $r r f-1$ mutant phenotype shows robust somatic expression, with prominent cytoplasmic and weak nuclear localization (Aoki et al., 2007). Despite their different expression patterns, the $r r f-1$ gene is encoded directly downstream of ego- 1 in an operon (Mangone et al., 2010).

Because exo-RNAi produces WAGO 22G RNAs to mediate transcript knockdown (Sijen et al., 2001; Pak and Fire, 2007; Sijen et al., 2007), the RNAi sensitivity phenotypes associated with loss of RRF-1 versus EGO-1 reveal their respective contributions to the WAGO pathway. RRF-1 is required in somatic tissues for 22G RNA accumulation and therefore exo-RNAi (Sijen et al., 2001; Gent et al., 2010; Vasale et al., 2010), whereas no role for EGO-1 is detected during RNAi in the soma (Smardon et al., 2000; Sijen et al., 2001). Accordingly, 22G RNAs mapping to ERGO-1 class 26G RNA targets are largely RRF-1-dependent (Vasale et al., 2010). In the germline, however, EGO-1 and RRF-1 are partially redundant for WAGO 22G RNA biogenesis. The rrf-1 mutant germline is sensitive to exo-RNAi of germline transcripts (Sijen et al., 2001), revealing compensation by EGO-1. This explains why loss of RRF-1 does not recapitulate the ts sterility and other germline phenotypes associated with total compromise of WAGO 22G RNA target silencing. Loss of EGO-1 results in a partial germline Rde phenotype (Smardon et al., 2000). Interestingly, injection of ego-l dsRNA enhances the germline defects of an ego-1 null mutant (Smardon et al., 2000), suggesting the possibility that RRF-1 may be recruited, although insufficiently, to CSR-1 22G RNA biogenesis in the absence of EGO-1. EGO-1 and phenotypes associated with its loss are discussed further with CSR-1 22G RNAs, below (Section 22).

\subsection{RRF-1}

In a cell-free system for analyzing secondary siRNA production triggered by exo-RNAi, RRF-1 accounts for 90\% of RdRP activity (Aoki et al., 2007). Secondary siRNAs generated by immuno-purified GFP::RRF-1 complexes are complementary to a supplied RNA template, and production is inhibited by addition of RNA chain elongation terminator. RRF-1 also exhibits terminal transferase activity in cell extracts. 93\% of RdRP products generated by GFP::RRF-1 complexes show a 5' guanosine. This indicates that the 5' guanosine bias of 22G RNAs, and perhaps also 26G RNAs, reflects an RdRP preference for initiation of transcription with GTP. GFP::RRF-1 complexes show very low processivity when incubated with ribonucleotides and mRNA template, generating products 22-23 nt in length; this length is not imposed by DCR-1-mediated cleavage, as DCR-1 is not recovered in GFP::RRF-1 complexes. DRH-3 immuno-purifies with GFP::RRF-1 and is required for RdRP activity. GFP::RRF-1 complexes fail to generate RdRP products from long dsRNA template, but polyadenylated and non-polyadenylated mRNAs are equally effective templates in vitro; in cell extract, the non-polyadenylated template stimulates more robust RdRP activity. Neither in cell extract nor in vitro do GFP::RRF-1 complexes catalyze extension of an RNA primer complementary to a template, arguing against primer-dependent synthesis. In contrast, GFP::RRF-1 complexes successfully incorporate labeled GTP as the 5' nucleotide of RdRP products in vitro, demonstrating unprimed synthesis (Aoki et al., 2007). The 5' ends of these RdRP products are also sensitive to capping, suggesting these products show the 5' triphosphate of 22G RNAs generated in vivo (Pak and Fire, 2007). Unprimed synthesis also occurs in vivo, as secondary siRNAs triggered by a mismatch-containing primary siRNA show perfect complementarity to the endogenous target (Sijen et al., 2007). Furthermore, the rare secondary siRNAs that are generated 3' to the trigger sequence could not originate via extension of exo-siRNA-derived primers (Pak and Fire, 2007).

\section{2. $\mathrm{DRH}-3$}

As mentioned above (see Section 3.2), DRH-3 is an essential component of all known C. elegans RdRP modules (Duchaine et al., 2006; Aoki et al., 2007; Nakamura et al., 2007; Claycomb et al., 2009; Gu et al., 2009). DRH-3 is detected at all developmental stages and is not restricted to germline (Claycomb et al., 2009), but the $d r h-3$ transcript is classified as germline-intrinsic (Reinke et al., 2004), and $d r h-3$ mRNA levels are three-fold higher in young adult and adult than in larva (Nakamura et al., 2007). The most severe phenotypes associated with loss of DRH-3 result from compromise of the CSR-1 22G RNA pathway (Claycomb et al., 2009) and will be discussed below (Section 22). Nevertheless, partial loss of DRH-3 function results in phenotypes and target upregulation attributable to loss of WAGO 22G RNAs: point mutations in the helicase domain yield viable $d r h-3$ hypomorphic mutants that show variable germline and somatic Rde, Mut, ts sterile, and Him phenotypes as well as embryonic lethality possibly due to reduced CSR-1 22G and transposon 22G RNA accumulation (Gu et al., 2009). Additionally, reduction of $d r h-3$ by RNAi results in hypersensitivity to DNA-damaging agents (Nakamura et al., 2007). This phenotype could be the result of defective selection of repair templates because of chromosome 
segregation defects (CSR-1 pathway). Alternatively, it may be that $d r h-3$ mutant animals already experience an increase in DNA double-strand breaks because of defects in transposon silencing (WAGO pathway) and are therefore hypersensitive to DNA-damaging agents. Finally, DRH-3 could play a more direct role in DNA double-strand break repair. A sterile $d r h-3$ null mutant is insensitive to RNAi in germline but not soma (Duchaine et al., 2006), presumably due to rescue by maternal inheritance. Deep sequencing of this mutant using a 5' monophosphate-independent protocol shows dramatically reduced endo-siRNA reads mapping to protein-coding genes, pseudogenes, repetitive elements, and unannotated loci but intact microRNAs and 21U RNAs (Gu et al., 2009). Residual 22G RNAs detected in the $d r h-3$ null mutant map primarily to the 3 ' ends of transcripts, suggesting that the RdRP module may be loaded at transcript 3' ends, with DRH-3 possibly acting to promote sequential initiation of polymerization (Gu et al., 2009). Proposed roles for DRH-3 within the RdRP module include relaxing template secondary structure and promoting $22 \mathrm{G}$ RNA dissociation from the transcript for Argonaute loading (Gu et al., 2009).

\subsection{EKL-1}

EKL-1 is a Tudor domain protein (Robert et al., 2005) paralogous to ERI-5. Like ERI-5, EKL-1 interacts with DRH-3, but only ERI-5 interacts DCR-1 (Thivierge et al., 2012). This is consistent with 22G RNA biogenesis occurring through a DCR-1-independent mechanism (Aoki et al., 2007; Gu et al., 2009; Pavelec et al., 2009). The ekl-1 transcript is classified as germline-intrinsic (Reinke et al., 2004), but EKL-1 is detected at all developmental stages and does not show germline restriction (Claycomb et al., 2009). Like RRF-1/EGO-1 and DRH-3, EKL-1 is also required for $22 \mathrm{G}$ RNA biogenesis (Gu et al., 2009) and exhibits phenotypes characteristic of compromise of CSR and WAGO 22G RNA pathways. Loss of EKL-1 results in sterility as well as somatic and germline defects in exo-RNAi and related phenomena (Kim et al., 2005; Robert et al., 2005; Rocheleau et al., 2008; Claycomb et al., 2009).

\section{Triggering of WAGO 22G RNAs}

WAGO 22G RNAs serve as the critical amplification pathway upon which most primary small RNAs in $C$. elegans converge. Accordingly, WAGO 22G RNAs are generated from and target transcripts targeted by 26G RNAs, primary exo-siRNAs, RDE-1-scavenged small RNAs, and 21U RNAs. The requirements for triggering of WAGO 22G RNAs by each of these primary small RNA types are addressed below (Sections 9.1, 9.2, 9.3, and 9.4).

Secondary siRNAs in C. elegans were first identified during exo-RNAi as a population of small RNAs that are antisense to a target mRNA but not derived from the initial dsRNA trigger (Sijen et al., 2001). However, the existence of some means of amplification had been hypothesized several years prior. It had been noted that a few molecules of dsRNA are sufficient to deplete a cellular pool of target mRNAs, arguing against simple stoichiometric interaction and indicating the involvement of a catalytic and/or amplification mechanism (Fire et al., 1998; Montgomery and Fire, 1998). Although trigger dsRNA cleavage generates some measure of amplification, this was recognized as still insufficient to explain the potent silencing ability of exogenous dsRNA (Sijen et al., 2001). The essential contribution of RdRP activity to this amplification was subsequently established by demonstrating complete insensitivity of the $r r f-1$ mutant soma to injection of prepared siRNA duplexes (Sijen et al., 2001).

WAGO 22G RNAs are generated from spliced transcripts targeted by the triggering primary small RNAs (Pak and Fire, 2007). RdRP initiation appears to be non-random however as 22G RNAs show some phasing across a target transcript, and similar sets of 22G RNAs are produced in different transgenic lines (Sijen et al., 2007). 22G RNA biogenesis shows a limited degree of spreading from the target site of the primary small RNA (Sijen et al., 2001). During exo-RNAi, spreading occurs primarily 5' of the primary RNA target site with respect to the sense of the target transcript (Sijen et al., 2001; Pak and Fire, 2007; Sijen et al., 2007). ERGO-1 class 26G RNAs rarely target the first $\sim 100 \mathrm{nt}$ of a transcript, but 22G RNAs show robust coverage of this interval at these same targets (Vasale et al., 2010). Similarly, 21U RNAs trigger 22G RNA production primarily 5' of the target site (Lee et al., 2012). This may have implications for transgenic construct design, as transgenes encoding foreign sequence 3 ' to endogenous sequence are more likely to evade silencing by 21U RNAs (Shirayama et al., 2012); presumably, 5' spread of 22G RNA biogenesis from foreign to endogenous sequence could be detrimental, resulting in selection for evaders. The range of the spreading is relatively modest and may vary by primary small RNA abundance. Exo-RNAi appears to generate secondary siRNAs with a range of 100-180 nt 5' to the region targeted by dsRNA (Alder et al., 2003). 21U RNAs trigger production of 22G RNAs within the surrounding 40-100 nt (Bagijn et al., 2012; Lee et al., 2012). 


\subsection{Triggering by $26 \mathrm{G}$ RNAs}

As described above, 26G RNAs trigger robust production of WAGO 22G RNAs at target sites, and these secondary siRNAs are essential for target silencing (Conine et al., 2010; Gent et al., 2010; Vasale et al., 2010). However, loss of 26G RNAs does not significantly deplete 22G RNA levels for all loci (Gent et al., 2009; Gu et al., 2009; Gent et al., 2010; Vasale et al., 2010), indicating the existence of other primary triggers of WAGO 22G RNA biogenesis.

\subsection{Triggering by primary exo-siRNAs}

Competition between endo- and exo-RNAi implicates primary exo-siRNAs and the associated Argonaute RDE-1 in triggering 22G RNAs as well (Sijen et al., 2001; Duchaine et al., 2006; Lee et al., 2006; Yigit et al., 2006). Exo-RNAi triggers production of antisense secondary siRNAs that share all of the features and dependencies of endogenously triggered WAGO 22G RNAs (Sijen et al., 2001; Tijsterman et al., 2002a; Aoki et al., 2007; Pak and Fire, 2007; Sijen et al., 2007; Gu et al., 2009). Expression of a single hairpin-derived 22 nt siRNA (22siR) targeting the unc-22 mRNA 3' UTR reveals some of the requirements for RDE-1-triggered 22G RNA biogenesis (Sijen et al., 2007): a 22siR showing perfect target site complementarity depletes unc-22 mRNA levels by $50 \%$ and generates abundant secondary siRNAs upstream of the target site. A mutated 22siR bearing target site mismatches at positions 10-12 binds the unc-22 mRNA but does not trigger secondary siRNA biogenesis. Single mismatches at position 11 or position 21 weaken target silencing, but do not alter the range of secondary siRNAs triggered. The lack of an effect of mismatch at the putative cleavage site (position 11) on the distribution of secondary siRNAs suggests that RDE-1-mediated target cleavage is not necessary for triggering secondary siRNA production (Sijen et al., 2007), consistent with a restricted role RDE-1 cleavage activity in passenger strand removal (Steiner et al., 2009).

\subsection{Triggering by RDE-1-scavenged small RNAs}

In the absence of exogenous dsRNA, RDE-1 binds a variety of DCR-1 products; among these are microRNAs and dsRNA-derived siRNAs cleaved from endogenous hairpins or bidirectionally transcribed genomic regions (Corrêa et al., 2010). Some of these scavenged siRNAs initiate significant 22G RNA production at target loci: the Y47H10A.5 transcript generates abundant 22G RNAs triggered by miR-243-loaded RDE-1, for which a perfectly complementary target site $22 \mathrm{nt}$ in length appears on Y47H10A.5 transcript (Welker et al., 2007; Gu et al., 2009; Corrêa et al., 2010). Like primary exo-siRNAs, miR-243-mediated triggering of these $22 \mathrm{G}$ RNAs requires RDE-4 in addition to RDE-1 (Gu et al., 2009). In contrast, the chief microRNA pathway Argonaute ALG-1 does not trigger secondary siRNA biogenesis at target sites (Steiner et al., 2007); this may explain why microRNAs fail to elicit dramatic target knockdown despite the vast abundances of individual microRNAs relative to individual endo-siRNAs. It is important to note that $22 \mathrm{G}$ RNAs themselves are rarely bound by RDE-1 (Sijen et al., 2001), prohibiting triggering of further siRNA biogenesis from aberrant tertiary targets in trans. Similarly, 22G RNAs bound by WAGO Argonautes also do not appear to trigger further amplification or spreading at target sites: a sensor transgene with a single target site for an abundant $22 \mathrm{G}$ RNA shows confined loss of secondary siRNAs at the target site when the triggering 22G RNA is removed (Montgomery et al., 2012).

\subsection{Triggering by $21 U$ RNAs}

A fourth population of primary small RNAs converging on the WAGO 22G RNAs are 21U RNAs, the piRNAs of $C$. elegans. These small RNAs are bound by and require the PIWI clade Argonaute PRG-1 for accumulation and targeting (Batista et al., 2008; Das et al., 2008). 21U RNAs constitute a library of mismatch-tolerant sequences that are depleted for targeting of protein-coding genes but capable of silencing non-self transcripts by triggering WAGO 22G RNAs (Ashe et al., 2012; Bagijn et al., 2012; Lee et al., 2012; Shirayama et al., 2012). 21U RNA properties, biogenesis, and function are discussed in depth below. Initial studies identified $21 \mathrm{U}$ RNA-dependent secondary siRNAs (Batista et al., 2008; Das et al., 2008), but only recently were these established as WAGO 22G RNAs and the targeting requirements reported. 21U RNAs trigger 22G RNA biogenesis at engineered sensor targets with up to two target site mismatches and at endogenous targets with up to three or four mismatches (Bagijn et al., 2012; Lee et al., 2012). Seed site pairing, which plays an important role in microRNA-mediated target repression, may also be important for 21U RNA targeting. One study found that nearly perfect pairing is required at positions 2-8, with a maximum of one G-U wobble pair across the interval, to trigger 22G RNA biogenesis (Lee et al., 2012); however, another study found that transgenic 21U RNA sensor transcripts are effectively targeted regardless of the position of dinucleotide mismatches across the length of the 21U RNA 
Bagijn et al., 2012). Interestingly, 21U RNAs seem less effective at stimulating a secondary siRNA response, as less than $5 \%$ of target sites exhibiting perfect complementarity to $21 \mathrm{U}$ RNAs show unambiguous triggering of $22 \mathrm{G}$ RNAs (Bagijn et al., 2012; Lee et al., 2012). This may be in part due to the low abundances of many 21U RNA species, as levels of $22 \mathrm{G}$ RNAs triggered correlate with both $21 \mathrm{U}$ RNA abundance and degree of complementarity (Bagijn et al., 2012; Lee et al., 2012). Recombinant PRG-1 shows some slicing activity in vitro, but this activity is dispensable for triggering 22G RNA production as well as target silencing (Bagijn et al., 2012; Lee et al., 2012). Also in support of a cleavage-independent mechanism, 21U RNA target site mismatches at positions 10 and 11 do not abrogate 22G RNA triggering (Bagijn et al., 2012; Lee et al., 2012).

\section{Germline amplification of WAGO 22G RNAs in perinuclear Mutator foci}

The observation that some natural isolates of $C$. elegans are mutators (strains that have a high spontaneous germ line mutation rate) not only led to the use of these strains in genetic screens but also to research into the causal "mutagen", DNA transposons of the Tc1/mariner family, including Tc1 and Tc3. To understand how activity of these transposons is regulated, genetic screens were performed to identify mutants that display increased Tc1 or Tc3 transposition in the germline (Collins et al., 1987; Ketting et al., 1999; Vastenhouw et al., 2003). The description of the first mutator (Mut) gene identified, mut-7 (Ketting et al., 1999), and of the first genetic screen for exo-RNAi-defective (Rde) mutants (Tabara et al., 1999), provided the first evidence of a role for RNAi in transposon silencing in the germline: many Rde mutants displayed Mut phenotypes and many Mut mutants displayed Rde phenotypes. These and subsequent screens for related phenomena (Ketting et al., 1999; Vastenhouw et al., 2003; Grishok et al., 2005; Kim et al., 2005; Robert et al., 2005) recurrently identified members of a group of proteins that have now been implicated in formation of Mutator foci. These perinuclear processing compartments interact with RRF-1 and are required for WAGO 22G RNA amplification and target silencing in the germline (Phillips et al., 2012). The six proteins that assemble to form Mutator foci are MUT-16, MUT-7, MUT-8/RDE-2, MUT-2/RDE-3, MUT-15, and MUT-14. Consistent with their critical, non-redundant role in the WAGO 22G RNA pathway that silences transposons, loss of any of these proteins results in mutator (Mut), ts sterile, Him, and Rde phenotypes (Collins et al., 1987 Ketting et al., 1999; Tijsterman et al., 2002a; Vastenhouw et al., 2003; Chen et al., 2005; Tops et al., 2005; Gu et al., 2009; Phillips et al., 2012), although a mut-14 mutant carrying a missense mutation shows only a germline Rde phenotype (Tijsterman et al., 2002a; Phillips et al., 2012).

MUT-16 is a worm-specific protein containing proline-rich and glutamine/asparagine-rich regions (Vastenhouw et al., 2003). A translational MUT-16::GFP fusion protein is expressed broadly in cytoplasm and nuclei (Vastenhouw et al., 2003). MUT-16 is also required for accumulation of ERGO-1 class 26G RNAs, whereas other Mutator foci components are not strictly required (Zhang et al., 2011). MUT-7 is a 3' to 5' exonuclease (Ketting et al., 1999) that is conserved in animals (Phillips et al., 2012). A mut-7::gfp transcriptional fusion shows expression in many different cell types in larva and adult (Hunt-Newbury et al., 2007). MUT-8/RDE-2 contains no known domains and may only exist to recruit MUT-7 (Tops et al., 2005; Phillips et al., 2012). MUT-2/RDE-3 is a $\beta$ nucleotidyltransferase; mutations of conserved residues at the active site produce Rde phenotypes, suggesting polymerase activity is required for function (Chen et al., 2005). MUT-2/RDE-3 expressed in frog oocytes shows no polymerase activity, unlike homolog CDE-1 (Kwak and Wickens, 2007). MUT-15 contains no known domains. MUT-14 is a DEAD box RNA helicase (Tijsterman et al., 2002a).

\subsection{Dynamics of Mutator foci}

Germline Mutator foci are most prominent in the mitotic region and transition zone, showing perinuclear localization adjacent to $\mathrm{P}$ granules until late meiosis (Phillips et al., 2012). Somatic Mutator foci components are cytoplasmically localized but still required for efficient WAGO 22G RNA amplification and silencing function (Phillips et al., 2012), as most of the component proteins are required for exo-RNAi in both germline and soma (Ketting et al., 1999; Vastenhouw et al., 2003; Chen et al., 2005; Kim et al., 2005; Tops et al., 2005; Gu et al., 2009; Phillips et al., 2012). Failure to assemble Mutator foci in somatic tissues likely decreases the effectiveness of the components in promoting genome surveillance, as transposons efficiently silenced in germline can be active in the soma (Emmons and Yesner, 1984). Inheritance of maternal Mutator foci may be important for transposon suppression in offspring (Ketting et al., 1999).

\subsection{Assembly of Mutator foci}

Mutator foci may associate directly with the nuclear pore complex (Phillips et al., 2012), much like P granules (Pitt et al., 2000). However, Mutator foci are not required for $\mathrm{P}$ granule formation, nor are $\mathrm{P}$ granules required for Mutator focus assembly (Phillips et al., 2012). Consistent with the many associations of the nuclear pore with RNA 
processing bodies, nuclear pore complex proteins routinely emerge from screens for factors involved in RNAi (Vastenhouw et al., 2003; Kim et al., 2005). Mutator focus assembly is a regulated process: MUT-16, which contains a Q/N-rich domain that may mediate protein-protein interactions, recruits MUT-2/RDE-3, MUT-15, MUT-14, and MUT-8/RDE-2, which itself recruits MUT-7 (Tops et al., 2005; Phillips et al., 2012). In the absence of MUT-16, the other five components do not interact (Phillips et al., 2012). Intriguingly, formation of Mutator foci appears not to require the presence of WAGO 22G RNAs, as loss of DRH-3, EKL-1, or EGO-1 and RRF-1 simultaneously or alone does not affect their formation, nor does loss of 26G RNAs, CSR-1 22G RNAs, or several individual WAGO Argonautes (Phillips et al., 2012).

\subsection{Function of Mutator foci}

Mutator foci serve as amplification centers for WAGO 22G RNAs in the germline (Phillips et al., 2012). mut-15, mut-16, and mut-2/rde-3 mutants show defects in secondary siRNA accumulation in response to exo-RNAi (Sijen et al., 2007), and deep sequencing of mut-2, mut-7, and mut-16 mutants reveals general defects in accumulation of WAGO 22G RNAs (Gu et al., 2009; Zhang et al., 2011). Somatic X-cluster 22G RNAs are depleted in mut-15, mut-2/rde-3, mut-7, and mut-16 mutants (Zhang et al., 2011; Phillips et al., 2012). A mut-14 mutant shows intact X-cluster 22G RNAs, but loss of germline 22G RNAs (Phillips et al., 2012). Therefore, the germline and somatic Rde phenotypes of Mutator foci mutants reflect their respective capacities to support 22G RNA biogenesis. In Mutator foci mutants, however, loss of WAGO 22G RNAs is incomplete, and depletion does not appear proportionate for all species. For example, the targets showing greatest 22G RNA depletion upon loss of MUT-16, MUT-2, or MUT-7 are those that generate very high levels of 22G RNAs (Gu et al., 2009; Zhang et al., 2011; Phillips et al., 2012). Furthermore, MUT-2/RDE-3 is required for full target knockdown during dsRNA feeding, but not when dsRNA is transgenically expressed (Chen et al., 2005). These data suggest that Mutator foci are most important for heavy siRNA amplification and are less critical when primary small RNA triggers are abundant. The mechanisms by which Mutator foci promote WAGO 22G RNA amplification are not known, although recruitment of RdRP to target transcripts is likely involved. Germline RRF-1 localizes exclusively to Mutator foci, whereas EGO-1 localizes both to P granules and Mutator foci (Claycomb et al., 2009; Phillips et al., 2012), reflecting the dual function of EGO-1 in the WAGO and CSR-1 22G RNA pathways (Gu et al., 2009). The adjacency of Mutator foci and $\mathrm{P}$ granules suggests possible interaction between the two (Phillips et al., 2012). P granules contain ALG-3 and PRG-1 (Batista et al., 2008; Wang and Reinke, 2008; Conine et al., 2010), two Argonautes that require WAGO 22G RNA-mediated amplification to effect target silencing (Das et al., 2008; Gu et al., 2009; Conine et al., 2010; Bagijn et al., 2012; Lee et al., 2012). When a nascent mRNA passes through the nuclear pore, the presence of the hydrophobic $\mathrm{P}$ granule may retard its diffusion to enhance recognition by regulatory molecules such as loaded Argonautes (Sheth et al., 2010). Once a target transcript enters a P granule and is recognized by a primary small RNA Argonaute, the adjacent Mutator focus may engage the transcript for secondary siRNA amplification. Notably, CSR-1 22G RNAs do not accumulate to high levels or trigger gene silencing (Claycomb et al., 2009; Gu et al., 2009) and do not require Mutator foci for amplification, as a mut-16 mutant does not show depletion of 22G RNAs targeting a validated set of CSR-1 22G RNA pathway targets (Zhang et al., 2011).

\section{Additional amplification of WAGO 22G RNAs by RDE-10/RDE-11}

The RDE-10/RDE-11 protein complex amplifies 22G RNAs and potentiates degradation of the targets that serve as their templates (Yang et al., 2012; Zhang et al., 2012). RDE-10 and RDE-11 are worm-specific genes (Yang et al., 2012). WormBase reports RDE-10 as containing a Maelstrom domain, which adopts an RNase $\mathrm{H}$ fold that may confer nuclease or RNA-binding functionality. RDE-11 contains a RING-type zinc finger domain (Yang et al., 2012). Mutation of a key zinc-coordinating residue in RDE-11 abrogates its interaction with RDE-10 in vitro and results in failure to rescue $r d e-11$ mutant phenotypes (Yang et al., 2012). Thus, zinc binding facilitates the formation of the RDE-10/RDE-11 complex, possibly by promoting folding of RDE-11.

\subsection{RDE-10/RDE-11 amplifies 22G RNAs triggered by exo-RNAi and ERGO-1}

The RDE-10/RDE-11 complex appears to play a significant role in amplification of WAGO 22G RNAs triggered by exo-RNAi (Yang et al., 2012; Zhang et al., 2012). The absence of RDE-10 or RDE-11 results in a partial germline and somatic Rde phenotype, but no fertility defect or Him phenotype (Yang et al., 2012; Zhang et al., 2012). The exo-RNAi deficiency of these mutants is dosage-sensitive and results from impaired accumulation of secondary WAGO 22G RNAs: loss of RDE-10/RDE-11 depletes exo-RNAi-triggered 22G RNAs by $~ 80 \%$ (Yang et al., 2012; Zhang et al., 2012). ERGO-1 class 26G RNAs also variably require RDE-10/RDE-11 for maximal amplification of secondary siRNAs and target silencing. ERGO-1 is detected in immuno-purified RDE-10 
complexes (Yang et al., 2012; Zhang et al., 2012), and embryonic 22G RNAs targeting ERGO-1 26G RNA targets are depleted by more than half upon loss of RDE-10 (Zhang et al., 2012). 22G RNAs are most abundant in the germline (Gu et al., 2009; Gent et al., 2010), and some primary small RNAs are restricted to germline tissues (Batista et al., 2008; Das et al., 2008; Wang and Reinke, 2008; Conine et al., 2010). The more pronounced effects of RDE-10/RDE-11 on exo-RNAi and ERGO-1-mediated silencing therefore suggest that the RDE-10/RDE-11 complex may be particularly important in somatic 22G RNA amplification. Indeed, the somatic X-cluster and Y47H10A.5 22G RNAs triggered by ERGO-1 and RDE-1, respectively, showed strong dependence on RDE-10 and RDE-11 for accumulation (Zhang et al., 2012). In comparison, accumulation of a germline-enriched, ERGO-1-independent 22G RNA targeting T01A4.3 (Duchaine et al., 2006) does not require RDE-10/11 (Zhang et al., 2012), nor apparently do many other WAGO 22G RNAs, microRNAs, 21U RNAs, 26G RNAs, primary exo-siRNAs, or CSR-1 22G RNAs (Yang et al., 2012; Zhang et al., 2012). Perhaps the RDE-10/RDE-11 complex supports WAGO 22G RNA amplification in the soma, in the absence of perinuclear Mutator foci.

\subsection{RDE-10/RDE-11-mediated target degradation}

In addition to amplifying $22 \mathrm{G}$ RNAs, the RDE-10/RDE-11 complex may directly promote target deadenylation and degradation. RDE-10 binds mRNAs targeted by exo-RNAi, even in the absence of RDE-11 (Yang et al., 2012). This binding activity is lost in the absence of RDE-1, but intact in the absence of RRF-1, indicating that primary exo-siRNAs are guiding association of RDE-10/RDE-11 with mRNA targets independently of secondary 22G RNAs (Yang et al., 2012). After recognition, RDE-10-bound mRNAs are deadenylated and degraded in an RDE-11-dependent manner (Yang et al., 2012). Targets undergoing RDE-10/RDE-11-mediated degradation retain 5' caps, providing a transiently stable substrate for continued secondary siRNA production (Yang et al., 2012). Thus, the RDE-10/RDE-11 complex may promote secondary siRNA biogenesis by binding exo-RNAi targets to promote RRF-1 binding as deadenylation and degradation occur (Yang et al., 2012). The RDE-10/RDE-11-mediated degradation pathway likely acts in parallel to target silencing mediated by somatic nuclear Argonaute NRDE-3 and other WAGO Argonautes, as many NRDE-3-bound 22G RNAs are intact in the absence of RDE-10/RDE-11 (Yang et al., 2012; Zhang et al., 2012). In somatic exo-RNAi in particular, the RDE-10/RDE-11 complex and NRDE-3 may represent the two main pathways for target silencing: rde-10, rde-11, and $n r d e-3$ mutants all show partial sensitivity to $u n c-22$ dsRNA feeding, but loss of RDE-10 or RDE-11 in a $n r d e-3$ mutant background results in complete insensitivity to unc-22 dsRNA and a defect in unc-22 mRNA knockdown as profound as observed upon complete loss of exo-RNAi in the rde-l mutant (Yang et al., 2012).

\subsection{RSD-2, RSD-6, and/or HAF-6 may potentiate RDE-10/RDE-11 function}

RSD-2 and Tudor domain protein RSD-6 are worm-specific proteins; RSD-2 contains no annotated domains (Tijsterman et al., 2004) HAF-6 is a half-molecule ATP-binding cassette transporter detected with a reticular pattern in intestinal, muscle, and germline cells, with perinuclear localization in germline (Sundaram et al., 2006). Like rde-10 and $r d e-11$ mutants, $r s d-2$, rsd-6, and haf-6 mutants also show dosage-sensitive Rde phenotypes (Tijsterman et al., 2004; Sundaram et al., 2006; Han et al., 2008) due to defects in WAGO 22G RNA accumulation (Han et al., 2008; Zhang et al., 2012). These three proteins also regulate accumulation and target silencing of a subset of WAGO 22G RNAs, many of which are ERGO-1- and RDE-10/RDE-11-dependent (Zhang et al., 2012). RSD-2, RSD-6, and HAF-6 are all required for accumulation of X-cluster 22G RNAs (Zhang et al., 2012). The shared dosage-sensitive Rde phenotype and functional overlap of RDE-10/RDE-11 and RSD-2, RSD-6, and HAF-6 suggests the possibility of a common complex. Supporting this, RSD-2 interacts with both RDE-10 and RSD-6 (Tijsterman et al., 2004; Zhang et al., 2012). However, loss of RSD-2 also results in a Mut phenotype at $25^{\circ} \mathrm{C}$, and loss of RSD- 6 causes ts sterile and Him phenotypes (Han et al., 2008). These more severe WAGO 22G RNA pathway phenotypes indicate that RSD-2 and RSD-6 may play more critical or additional roles in WAGO 22G RNA accumulation or function. The perinuclear germline localization of HAF-6 (Sundaram et al., 2006) suggests that HAF-6 could also interact with Mutator foci, and there is some evidence for genetic interaction between HAF-6 and Mutator foci components: double heterozygous mut-8/rde-2(+/-); mut-7(+/-) mutants show impaired exo-RNAi, and heterozygosity for a haf-6 mutation in either a mut-8/rde-2(+/-) or mut-7(+/-) heterozygous background also results in defects in exo-RNAi (Sundaram et al., 2006).

\section{WAGO 22G RNA Argonaute loading}

Early analysis of $C$. elegans Argonautes revealed significant expansion of the protein family (Grishok et al., 2001). A large subset of these cluster phylogenetically into a WAGO clade that is roughly equally distant from the conserved PIWI and AGO clades (Yigit et al., 2006). Included in these are CSR-1, the Argonaute that mediates the CSR-1 22G RNA pathway (Claycomb et al., 2009), and related protein C04F12.1. Also variably included is the 
divergent RDE-1, the first C. elegans Argonaute to be linked to RNAi (Tabara et al., 1999). The other 12 Argonaute proteins in this clade constitute the WAGO Argonautes of the WAGO 22G RNA pathway. These 12 Argonautes bind and stabilize WAGO 22G RNAs semiredundantly to mediate target silencing. Simultaneous loss of all 12 Argonautes in the MAGO12 Argonaute mutant results in penetrant ts sterile, Him, and Rde phenotypes and massive depletion of 22G RNAs that are not dependent on CSR-1 (Claycomb et al., 2009; Gu et al., 2009). The ts sterility of the MAGO12 mutant can only be partially rescued by wild type sperm, likely because loss of 21U RNAs, one of the triggers of 22G RNA biogenesis, results in ts fertility defects in female germline as well as male (Wang and Reinke, 2008). These WAGO-dependent $22 \mathrm{G}$ RNAs are also globally depleted in mut-7 and mut-2/rde-3 mutants (Gu et al., 2009), reflecting the contribution of Mutator foci components to WAGO 22G RNA accumulation (Phillips et al., 2012).

Although the phylogenetic relationships between the Argonautes can vary depending on whether full-length protein or specific domains were used for phylogenetic analysis, the 12 WAGO Argonautes of the cognate $22 \mathrm{G}$ RNA pathway (Grishok et al., 2001; Yigit et al., 2006; Montgomery et al., 2012) are usually clustered into three subclades referred to by phylogenetic branch: Branch 1 comprises WAGO-1 (R06C7.1), WAGO-2 (F55A12.1), WAGO-3 (PPW-2/Y110A7A.18), WAGO-4 (F58G1.1), WAGO-5 (ZK1248.7); Branch 2 comprises WAGO-6/8 (SAGO-2/F56A6.1), WAGO-7 (PPW-1/C18E3.7), WAGO-8/6 (SAGO-1/K12B6.1); and Branch 3 comprises WAGO-9 (HRDE-1/C16C10.3), WAGO-10 (T22H9.3), WAGO-11 (Y49F6A.1), WAGO-12 (NRDE-3/R04A9.2) (Gu et al., 2009). wago-1, wago-2, wago-3/ppw-2, and wago-5 transcripts are classified as germline-intrinsic (Reinke et al., 2004). wago-4 and wago-9/hrde-1 transcripts are classified as oogenesis-enriched, whereas wago-10 is spermatogenesis-enriched (Reinke et al., 2004). The wago-12/nrde-3 transcript is classified as mixed oogenesis-somatic (Reinke et al., 2004). The others are not assigned germline enrichment classifications (Reinke et al., 2004). WAGO-12/NRDE-3 and WAGO-9/HRDE-1 will be discussed in greater detail below in the context of the nuclear RNAi pathway.

Loss of single WAGO Argonautes can cause mild defects in exo-RNAi sensitivity and germline transposon silencing, such as the germline Rde phenotype observed in a natural C. elegans isolate (CB4856) that lacks functional WAGO-3/PPW-2 (Tijsterman et al., 2002b; Vastenhouw et al., 2003; Yigit et al., 2006). Simultaneous loss of multiple WAGO Argonautes within the same phylogenetic branch produces more significant impairment. The MAGO strain lacks Branch 2 and WAGO-4 Argonautes and shows a somatic and germline Rde phenotype and a ts fertility defect (Yigit et al., 2006). The Quadruple mutant lacks all of the Branch 1 Argonautes but WAGO-1 and shows an Rde phenotype (Gu et al., 2009). Similarly, WAGO Argonautes bind and stabilize WAGO 22G RNAs semiredundantly. In exo-RNAi of the unc-22 transcript, Branch 2 Argonautes bind secondary siRNAs redundantly, and overexpression of any Branch 2 Argonaute rescues the RNAi defect of the MAGO strain, which otherwise fails to accumulate unc-22 secondary siRNAs (Yigit et al., 2006). Still, particular 22G RNAs in germline and soma show different dependencies upon different WAGO Argonautes. The Quadruple mutant, while showing a germline Rde phenotype, still generates wild-type levels of a germline F37D6.3 22G RNA, whereas loss of the remaining Branch 1 Argonaute, WAGO-1, severely depletes this species (Gu et al., 2009). Loss of all five Branch 1 Argonautes in the Quintuple mutant results in dramatic reduction of germline 22G RNAs, whereas a MAGO+2 mutant lacking Branch 2, WAGO-3, and WAGO-4 Argonautes (Gu et al., 2009) shows near wild type levels of germline 22G RNAs, but loss of a somatic Y47H10A.5 22G RNA triggered by RDE-1 (Gu et al., 2009).

\section{WAGO 22G RNA-mediated post-transcriptional gene silencing}

Early evidence suggested that exo-RNAi occurred primarily through a post-transcriptional silencing mechanism. Injection of dsRNA segments corresponding to intron and promoter sequences does not elicit interference (Fire et al., 1998), and injection of dsRNA targeting a transcript encoded in an operon generally does not decrease levels of transcripts encoded downstream of the target within the operon appreciably (Montgomery et al., 1998). Little progress has been made in understanding cytoplasmic, post-transcriptional target silencing by WAGO 22G RNAs. It is unlikely to involve Argonaute-mediated target cleavage, as WAGO Argonautes lack the catalytic triad residues required for slicer activity (Yigit et al., 2006); furthermore, target cleavage induced by introduction of 5' triphosphorylated siRNAs into cell lysate is predominantly mediated by CSR-1 (Aoki et al., 2007), which is not involved in the WAGO 22G RNA pathway (Claycomb et al., 2009; Gu et al., 2009). This suggests that other nucleases may be recruited to the target mRNA to mediate mRNA degradation in the WAGO pathway. It is possible that RDE-10/RDE-11-mediated target deadenylation and degradation represents the main post-transcriptional mechanism for target silencing (Yang et al., 2012). In the germline, post-transcriptional target silencing may occur at P granules, as GFP::WAGO-1 is expressed in the germline and localizes to perinuclear P granules (Gu et al., 2009), like Argonautes ALG-3, PRG-1, and CSR-1 (Batista et al., 2008, Wang and Reinke, 2008; Claycomb et al., 2009; Conine et al., 2010). Earlier data connecting the nonsense-mediated decay (NMD) pathway with exo-RNAi likely resulted from the presence of the mut-16(mg461) mutation in the mutant strains of 
smg-2, smg-5, and smg-6 genes, which encode NMD pathway proteins (Zhang et al., 2011). This explanation is consistent with an initial report that failed to demonstrate a role for the SMG proteins in exo-RNAi (Montgomery et al., 1998), although a transgenic system for studying transcriptional gene silencing showed impairment of repression upon depletion of $s m g-5$ by RNAi (Grishok et al., 2005).

\section{WAGO 22G RNA-mediated transcriptional gene silencing: The nuclear RNAi pathway}

\subsection{Early evidence for transcriptional gene silencing}

A complex of MES-2, MES-3 and MES-6 is functionally analogous to the Polycomb-repressive complex 2 (PRC2), a complex that catalyzes H3K27 trimethylation, a repressive chromatin mark. MES-4 generates H3K36me3 marks. MES-2, MES-3, MES-6, and MES-4 are required for germline silencing of high-copy arrays (Kelly and Fire, 1998), and silenced arrays carry histone modifications consistent with a heterochromatinized state (Kelly et al., 2002). A screen for factors involved in cosuppression, silencing in trans of an endogenous locus in response to a cognate transgenic array, identified numerous factors that regulate chromatin structure and transcription (Robert et al., 2005). In this system, silencing occurs at the transcriptional level, as relieving cosuppression through loss of MUT-16 or MUT-8/RDE-2 results in proportional increases in levels of both spliced and unspliced transcripts (Robert et al., 2005). Another transgene was found to show transcriptional silencing upon dsRNA feeding that occurs through a mechanism that requires both RNAi factors and proteins involved in chromatin regulation (Grishok et al., 2005). Transcriptional silencing of this transgene correlates with decreased RNA polymerase (Pol) II occupancy as well as increased $\mathrm{H} 4$ histone acetylation. Furthermore, this transgene shows spontaneous silencing upon loss of RRF-3 (Grishok et al., 2005), suggesting convergence of endo- and exo-RNAi upon a common a transcriptional silencing pathway.

\subsection{Early evidence for heritable and multigenerational gene silencing}

The effects of dsRNA injection are often observed in progeny, but rarely heritable beyond the first generation (Fire et al., 1998; Montgomery et al., 1998), suggesting the existence of an epigenetic heritable interfering agent of limited longevity. However, hermaphrodites injected with dsRNA targeting transcripts expressed in the maternal germline show multigenerational inheritance of silencing mediated by a dominant factor that can be inherited through either sperm or oocyte (Grishok et al., 2000). Similarly, a germline-expressed transgene transcriptionally silenced by cosuppression takes several generations to recover expression after relief of cosuppression (Robert et al., 2005). In contrast, transcriptional silencing of a transgene expressed in the soma is not inherited (Grishok et al., 2005). These data suggest the existence of two different forms of inherited RNAi: single-generation inheritance of somatic RNAi and multigenerational inheritance of germline RNAi.

\subsection{NRDE-3 and HRDE-1 WAGO Argonautes mediate nuclear RNAi}

Two WAGO Argonautes, WAGO-12/NRDE-3 and WAGO-9/HRDE-1, mediate somatic and germline nuclear RNAi, respectively, through what is referred to as the Nrde (Nuclear RNAi-defective) pathway (Figure 3). Although both NRDE-3 and HRDE-1 were reported to be completely dispensable for RNAi-mediated knockdown of pos-1 or let-2 (Yigit et al., 2006), these Branch 3 WAGO Argonautes appear to be the sole mediators of transcriptional gene silencing. As WAGO Argonautes, neither has an intact catalytic triad conferring slicer activity (Yigit et al., 2006), but each contains a bipartite nuclear localization signal (NLS) and shows predominantly nuclear localization (Guang et al., 2008; Ashe et al., 2012; Buckley et al., 2012). NRDE-3 and HRDE-1 are triggered by WAGO 22G RNA binding to enter the nucleus and associate with nascent pre-mRNA targets, where they recruit NRDE-2, NRDE-1, and NRDE-4 to inhibit Pol II elongation and deposit the repressive H3K9me3 chromatin mark (Guang et al., 2008; Guang et al., 2010; Burkhart et al., 2011; Burton et al., 2011; Ashe et al., 2012; Buckley et al., 2012; Gu, S.G., et al. 2012; Luteijn et al., 2012; Shirayama et al., 2012). The two Argonautes appear to use common silencing mechanisms and machinery (Burkhart et al., 2011; Ashe et al., 2012; Buckley et al., 2012; Luteijn et al., 2012), but their protein expression patterns are distinct: a rescuing GFP::NRDE-3 translational fusion protein shows expression in most somatic cells after the $~ 80$-cell embryo stage (Guang et al., 2008), whereas GFP::HRDE-1 is expressed in male and female germ cells (Buckley et al., 2012; Shirayama et al., 2012). Consequently, NRDE-3 mediates somatic nuclear RNAi, and HRDE-1 mediates germline nuclear RNAi. 


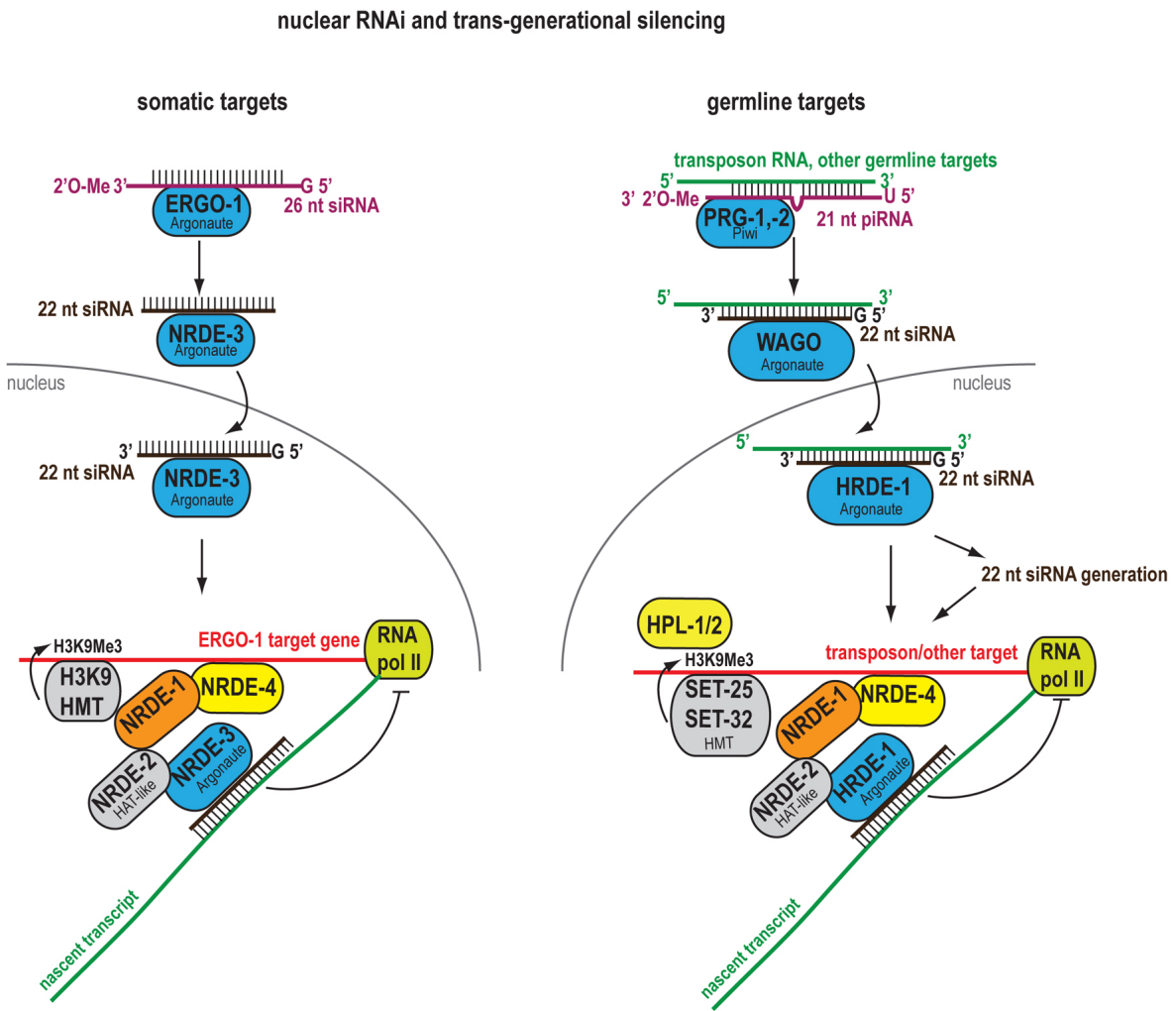

Figure 3. Nuclear RNAi pathways required for trans-generational silencing. Two pathways, silencing somatic targets and germline targets, converge on the same NRDE proteins but require distinct nuclear Argonaute proteins: cytoplasmic secondary 22G siRNAs associate with NRDE-3 in the soma and with HRDE-1 in the germline. The Argonaute:siRNA complex translocates to the nucleus where it recruits NRDE- 2 and NRDE-1 to the nascent pre-mRNA. NRDE-1 also associates with chromatin in a NRDE-4-dependent manner. NRDE-1 and NRDE-4 promote histone H3K9 trimethylation by histone methyltransferases and thus inhibit transcription. In addition, the NRDE proteins inhibit RNA polymerase II. Nuclear RNAi is heritable across generations: silencing signals, most likely siRNAs generated in the germline of the parent, are inherited and direct H3K9 trimethylation in the offspring.

\subsection{NRDE-3 collaborates with other WAGO Argonautes to silence somatic targets}

NRDE-3-mediated silencing is not always a significant component of the silencing response triggered by exo-RNAi or primary endogenous small RNAs (Guang et al., 2008; Guang et al., 2010). While NRDE-3 likely does not play an important role in silencing of cytoplasmic mRNAs (Guang et al., 2008), it is required for exo-RNAi of somatic nuclear-localized mRNAs including polycistronic pre-mRNAs such as lin-15a-lin15b and lir-1-lin-26 (Guang et al., 2008). Loss of $n r d e-3$ also results in increased pre-mRNA and mRNA levels of select endogenous targets (Guang et al., 2008); however, a majority of NRDE-3 targets show far less upregulation in the absence of NRDE-3 than in the absence of RRF-1/EGO-1, DRH-3, or EKL-1 (Gu et al., 2009). This indicates that other somatic Argonautes such as SAGO-1 and SAGO-2 (Yigit et al., 2006) collaborate with NRDE-3 to mediate WAGO 22G-RNA target silencing, potentially through the RDE-10/RDE-11 complex (Yang et al., 2012; Zhang et al., 2012). Accordingly, loss of NRDE-3 abrogates somatic nuclear RNAi entirely, but does not result in a classical somatic Rde phenotype (Guang et al., 2008). NRDE-3 is also required for single-generation inheritance of somatic target silencing triggered by exo-RNAi; in progeny, NRDE-3 promotes continued accumulation of secondary WAGO 22G RNAs to reestablish H3K9me3 marks (see Section 18) (Burton et al., 2011).

\subsection{HRDE-1 transgenerationally silences diverse targets to promote germline immortality}

In contrast to NRDE-3, HRDE-1 engages the Nrde pathway in germ cells to direct silencing that can be inherited over many generations (also called RNAe for RNA-induced epigenetic silencing) (Ashe et al., 2012; Bagijn et al., 2012; Buckley et al., 2012; Gu, S.G., et al., 2012; Luteijn et al., 2012; Shirayama et al., 2012). This transgenerational silencing can be initiated by exo-RNAi to establish transcript knockdown that is stable for several generations without additional trigger exposure (Ashe et al., 2012; Buckley et al., 2012; Gu, S.G., et al., 2012). Endogenous small RNA pathways also robustly engage germline nuclear RNAi. 22G RNAs bound by HRDE-1 and WAGO-1 largely overlap (Shirayama et al., 2012), affirming that nuclear and cytoplasmic WAGO Argonautes share 
a common set of siRNA cofactors and targets. These include WAGO 22G RNAs triggered by 21U RNAs and 26G RNAs that maintain germline integrity. In particular, 21U RNAs encode an epigenetic memory of non-self critical for genome surveillance (Ashe et al., 2012; Bagijn et al., 2012; Lee et al., 2012; Shirayama et al., 2012). These fertility-promoting small RNAs associate with HRDE-1 to initiate transgenerational silencing of targets that pose a threat to germline integrity; accordingly, loss of HRDE-1 or downstream Nrde factors causes progressive target desilencing and emergence of pleiotropic germline defects including a Him phenotype, production of nonfunctional gametes, and failure to generate mature oocytes or sperm in late generations at $25^{\circ} \mathrm{C}$ (Buckley et al., 2012). Effectively, loss of HRDE-1 results in a Mrt, or mortal germline, phenotype. While this phenotype is shared by mutants of downstream Nrde pathway members (NRDE-2, NRDE-1, and NRDE-4), it is not observed with loss of the somatic nuclear Argonaute NRDE-3 (Buckley et al., 2012). After engaging HRDE-1 to initiate transgenerational silencing, the triggering primary small RNA may not be required, as suggested by the dispensability of PRG-1 for maintenance of transgene silencing initiated by 21U RNAs (Ashe et al., 2012; Lee et al., 2012; Luteijn et al., 2012; Shirayama et al., 2012). For silencing of some targets, however, HRDE-1 and the Nrde pathway collaborate with other, likely cytoplasmic WAGO Argonautes: some transgenes are incompletely silenced by HRDE-1 and show increased desilencing upon loss of additional Argonautes including WAGO-10 (Shirayama et al., 2012). Additional requirements for transgenerational silencing are discussed further below.

\section{NRDE-3 target recognition}

Like other WAGO Argonautes, NRDE-3 binds WAGO 22G RNAs. Immuno-purified NRDE-3 complexes contain primarily 5' triphosphorylated $\sim 22$ nt endo-siRNAs (Guang et al., 2008). Consistent with production by a cytoplasmic RdRP acting on spliced transcripts, NRDE-3-associated siRNAs map to exonic sequences, even when RNAi is triggered by dsRNA containing introns (Guang et al., 2008). NRDE-3 primarily binds somatic 22G RNAs triggered by ERGO-1 class 26G RNAs, such as E01G4.5 22G RNAs, but also binds secondary siRNAs during exo-RNAi targeting a somatic transcript (Guang et al., 2008; Gent et al., 2010; Guang et al., 2010). Due to redundancy among the WAGO Argonautes, loss of NRDE-3 does not significantly impair stability of these $22 \mathrm{G}$ RNAs (Guang et al., 2008).

Upon binding a cytoplasmic WAGO 22G RNA, NRDE-3 translocates to the nucleus to execute target silencing (Guang et al., 2008). Nuclear localization is required for NRDE-3 function: an NLS-defective NRDE-3 mutant protein that binds siRNAs at wild type levels but does not redistribute to the nucleus fails to rescue nrde-3 mutant phenotypes (Guang et al., 2008). siRNA binding is required for translocation to the nucleus, as mutating the NRDE-3 PAZ domain to abrogate siRNA binding also results in failure to localize to the nucleus (Guang et al., 2008). Similarly, loss of siRNA cofactors abolishes nuclear localization. As NRDE-3 binds primarily somatic $22 \mathrm{G}$ RNAs triggered by ERGO-1 class 26G RNAs, GFP::NRDE-3 is cytoplasmic in eri-1, ergo-1, mut-7, rde-4, mut-2, eri-9, eri-6, and dcr-1(mg375) helicase mutants (Guang et al., 2008; Pavelec et al., 2009; Fischer et al., 2011). The nuclear localization of GFP::NRDE-3 is rescued upon introduction of dsRNA in an eri-1 mutant, but not an rde-4 mutant, which cannot initiate exo-RNAi (Guang et al., 2008); this suggests that siRNA binding is necessary and sufficient for nuclear redistribution of nuclear Argonautes.

After translocation to the nucleus, the loaded WAGO 22G RNA directs NRDE-3 to associate with unspliced target pre-mRNA (Guang et al., 2008). NRDE-3 targeting recognition appears to require very high sequence complementarity (Montgomery et al., 2012): A reporter transgene with a single target site for an X-cluster 22G RNA is efficiently silenced in a wild type background and shows primarily NRDE-3-dependent silencing. Silencing is abrogated by mismatches at positions 1-3, 4-5, 12-14, or deletion of a nucleotide at position 4. Silencing is impaired by mismatches at positions 9-11 or deletion or insertion of a nucleotide at position 13. Mismatches at position 13 or 20-22 are tolerated.

\section{The downstream nuclear RNAi machinery}

The proteins NRDE-2, NRDE-1, and NRDE-4 mediate the transcription silencing effects of nuclear Argonautes. After NRDE-3 binds a WAGO 22G RNA, translocates to the nucleus, and recognizes a nascent pre-mRNA target, NRDE-3 associates with NRDE-2 to recruit NRDE-1 to the transcript and direct its deposition on chromatin through a mechanism that requires NRDE-4 (Guang et al., 2008; Guang et al., 2010; Burkhart et al., 2011). NRDE-2, NRDE-1, and NRDE-4 are also engaged by HRDE-1 to mediate nuclear RNAi in the germline (Ashe et al., 2012; Buckley et al., 2012; Luteijn et al., 2012). Accordingly, loss of any of these factors results in the same Mrt phenotype observed with loss of HRDE-1 (Buckley et al., 2012), as well as loss of sensitivity to nuclear exo-RNAi (Guang et al., 2010; Burkhart et al., 2011). 


\subsection{NRDE-2}

NRDE-2 is a predominantly nuclear-localized, evolutionarily conserved protein encoding a domain of unknown function, a Serine/Arginine-rich domain, and a HAT-like domain, a domain consisting of repeats of three aromatic residues that is found in several RNA processing proteins (Guang et al., 2010). Immuno-purified NRDE-2 complexes contain NRDE-3, but only when the NRDE-3 NLS is intact, indicating an exclusively nuclear interaction (Guang et al., 2010). NRDE-2 is dispensable for NRDE-3 to transport siRNAs from cytoplasm to nucleus and for NRDE-3 to associate with the target pre-mRNA (Guang et al., 2010). Within the nucleus, NRDE-3 recruits NRDE-2 to the targeted nascent transcript. It is interesting to note that siRNA counts appear to be elevated two-fold by loss of NRDE-2 (Gu, S.G., et al., 2012); possibly, failure to silence target transcripts in the nucleus via the Nrde pathway results in increased cytoplasmic transcript accumulation, enhancing WAGO 22G RNAs production by RdRPs.

\subsection{NRDE-1}

NRDE-1 is a nematode-specific, nuclear-localized protein containing no obvious protein domains (Burkhart et al., 2011). NRDE-1 is recruited by NRDE-2/NRDE-3 to pre-mRNA near the target site, where it is required for nuclear RNAi-mediated inhibition of transcription elongation; a nrde-1 mutant fails to show 5' transcription inhibition of lin-15b upon RNAi and survives RNAi targeting of the lir-1 polycistron in a sensitizing eri-1 mutant background (Burkhart et al., 2011). NRDE-1 also associates with chromatin at the targeted genomic locus in a NRDE-4-dependent manner and links nuclear RNAi to chromatin regulation by promoting H3K9 trimethylation at genomic sites targeted by WAGO 22G RNAs.

\subsection{NRDE-4}

NRDE-4 is a nematode-specific protein with a predicted bipartite NLS and no other obvious domains (Burkhart et al., 2011). NRDE-4 does not recruit NRDE-3, NRDE-2, or NRDE-1 to pre-mRNA but is required for recruitment of NRDE-1 to chromatin and therefore for transcriptional inhibition and $\mathrm{H} 3 \mathrm{~K} 9$ trimethylation (Burkhart et al., 2011).

\section{Nuclear RNAi inhibits transcriptional elongation and drives H3K9 trimethylation}

Nuclear RNAi terminates transcription and promotes establishment of a repressed chromatin state at genomic loci targeted by exo-RNAi as well as multiple loci targeted by endo-siRNAs and 21U RNAs (Burkhart et al., 2011; Buckley et al., 2012; Gu, S.G., et al., 2012; Luteijn et al., 2012; Shirayama et al., 2012). Nuclear RNAi requires interaction with target pre-mRNA, as targeting 5' to the 5' UTR does not result in silencing (Gu, S.G., et al., 2012). NRDE-3, NRDE-2, and NRDE-1 bind to pre-mRNA at the target site but are not detected in association with pre-mRNA 5' to the target site, presumably due to co-transcriptional splicing of nascent transcripts (Moore and Proudfoot, 2009; Guang et al., 2010; Burkhart et al., 2011). Nrde targeting inhibits transcription elongation, as Pol II occupancy increases near the target site but not at initiation sites (Guang et al., 2010). Nuclear run-on assays show Pol II inhibition occurring $\sim 2 \mathrm{~kb}$ downstream of the target site, correlating with decreased Pol II occupancy by chromatin immunoprecipitation 3' to the target site (Guang et al., 2010; Burkhart et al., 2011). Because transcription is inhibited, NRDE-3, NRDE-2, and NRDE-1 do not bind pre-mRNA sequences encoded 3' to the target site; however, Nrde factors appear to remain associated with pre-mRNA fragments after silencing (Guang et al., 2010; Burkhart et al., 2011).

Nrde pathway targeting results in enrichment of H3K9 trimethylation at genomic target sites that correlates with the transcription inhibition (Burkhart et al., 2011), consistent with the established repressive role of H3K9 methylation. Whereas NRDE-3 and NRDE-2 recruit NRDE-1 to the RNA target near or 5' to the target site, NRDE-1 requires NRDE-4 for chromatin interaction and associates with chromatin predominantly 3' to the site of RNAi (Burkhart et al., 2011). Accordingly, H3K9 trimethylation occurs across the gene but peaks 3' to the target site (Burkhart et al., 2011). The chromatin response to nuclear RNAi occurs at the subgenic level, as shifting the RNAi trigger site shifts the interval of H3K9me3 deposition (Gu, S.G., et al., 2012). During exo-RNAi-triggered nuclear RNAi, secondary siRNAs accumulate mainly at exonic regions proximal to the dsRNA trigger site, but both exons and introns show high H3K9me3 levels after targeting (Gu, S.G., et al., 2012).

Although many of the studies of NRDE-3 function in nuclear RNAi were conducted under exo-RNAi, NRDE-3-mediated nuclear RNAi is also important for transcriptional silencing triggered by a subset of endogenous small RNAs in the soma (Burkhart et al., 2011). As mentioned above (see Section 15), NRDE-3 binds somatic 
WAGO 22G RNAs, many of which are triggered by ERGO-1 class 26G RNAs (Guang et al., 2008; Gent et al., 2010; Guang et al., 2010). 22G RNAs targeting the ERGO-1 target E01G4.5 are abundantly bound by NRDE-3 (Guang et al., 2008) and direct chromatin modification at this locus through the somatic nuclear RNAi pathway (Burkhart et al., 2011). Loss of members of the ERI complex, NRDE-3, or downstream Nrde pathway factors results in a subtle but detectable increase in E01G4.5 transcription inhibition, increased transcript levels, and depletion of H3K9me3 marks at the E01G4.5 locus (Burkhart et al., 2011). The strong influence of NRDE-3 on H3K9me3 deposition at the E01G4.5 locus is uncommon, and levels of many ERGO-1 targets show little or no effect of loss of the somatic Nrde pathway (Burkhart et al., 2011), consistent with significant overlap between cytoplasmic and nuclear WAGO Argonautes in silencing of somatic targets (Gu et al., 2009). Nonetheless, in the absence of ERGO-1 class 26G RNAs, the somatic Nrde pathway can be heavily engaged by exo-RNAi, as indicated by the emergence of RNAi phenotypes for polycistronic transcripts in an eri-1 mutant (Bosher et al., 1999; Kennedy et al., 2004). In fact, exo-RNAi of some monocistronic transcripts also engages NRDE-3 in the absence of ERGO-1 class 26G RNAs, as exo-RNAi targeting $u n c-73$ and $d p y-13$ results in less severe phenotypes in an eri-1; nrde-3 double mutant than in an eri-1 mutant (Guang et al., 2008). While loss of ERGO-1 class 26G RNAs does sensitize the somatic nuclear RNAi system for phenotypic analysis, an eri-1 or equivalent mutant background is not necessary for the transcription- and chromatin-level effects of nuclear RNAi (Burkhart et al., 2011).

\section{NRDE-3 directs single-generation epigenetic inheritance of silencing}

Although NRDE-3 acts primarily in somatic cells, the silencing effects of somatic nuclear RNAi are transmissible for one generation (Burton et al., 2011). Exo-RNAi in the parental generation generates secondary siRNAs abundantly bound by NRDE-3; inheriting progeny also show high levels of NRDE-3-bound secondary siRNAs from embryo through adulthood, although these are not inherited into F2 embryos (Burton et al., 2011). In the absence of NRDE- 1 or NRDE-4, these secondary siRNAs are still inherited and associate with NRDE-3, but levels of NRDE-3-bound secondary siRNAs are not maintained throughout development (Burton et al., 2011). This indicates that somatic nuclear RNAi is not required for inheritance of secondary siRNAs but is required for continued expression of these siRNAs in progeny.

H3K9me3 marks established in the parental generation at RNAi target loci are lost in progeny during the embryo stage, possibly due to the absence of NRDE-3 in early embryogenesis (Guang et al., 2008; Burton et al., 2011). Silencing is then reestablished through NRDE-3 and downstream Nrde factors during development and strengthened to exceed the H3K9me3 levels in the parental generation (Burton et al., 2011). The mechanism is likely the same in progeny as in the parental generation as NRDE-3 siRNA binding and nuclear translocation are still required for inherited silencing (Burton et al., 2011). However, the increased H3K9me3 deposition in progeny suggests that the inheritance of a silencing signal may further promote the ability of nuclear RNAi to repress chromatin, possibly by enhancing coupling of siRNA generation and H3K9me3 deposition in progeny. Importantly, this reestablishment of silencing requires the activity of NRDE-3 in progeny: homozygous nrde-3 mutant progeny of heterozygous nrde-3 hermaphrodites subjected to exo-RNAi do not show inheritance of silencing (Burton et al., 2011). The converse has not been tested; it is not known whether somatic nuclear RNAi is required in the parental generation for RNAi inheritance and chromatin repression in progeny. Furthermore, it is as yet unclear whether somatic nuclear RNAi is required for establishment or maintenance of transcriptional silencing during RNAi inheritance in the soma.

Somatic nuclear RNAi in the parental generation directs siRNA expression and H3K9 trimethylation in inheriting progeny through one of two possible mechanisms. NRDE-3-bound secondary siRNAs may be directly deposited into germ cells to direct embryonic silencing in association with other WAGO Argonautes, then re-bind NRDE-3 to promote maintenance of siRNA expression through nuclear RNAi during development. Alternatively, a different silencing signal such as primary small RNAs may be deposited in germ cells to promote expression of siRNAs in progeny that bind NRDE-3 and re-engage nuclear RNAi (Burton et al., 2011). In support of the latter mechanism, NRDE-3-bound WAGO 22G RNAs do not appear to initiate their own amplification at target sites (Montgomery et al., 2012), suggesting that primary small RNA trigger may be required for secondary siRNA re-amplification in progeny. One likely possibility is that primary exo-siRNAs are directly inherited from hermaphrodites exposed to dsRNA. Indeed, $r d e-1$ transcript is abundant in hermaphrodite germline and detected at high levels in embryos of all stages (NEXTDB clone 26b3), suggesting that primary exo-siRNAs may be inherited bound to RDE-1 to trigger NRDE-3-mediated nuclear RNAi in inheriting progeny. Such a mechanism would explain why somatic nuclear RNAi is heritable for only a single generation. 


\section{HRDE-1 directs multigenerational epigenetic inheritance of silencing}

Like NRDE-3, HRDE-1 engages the Nrde downstream machinery to direct transcriptional silencing and H3K9me3 deposition at loci targeted by exo-RNAi and endogenous small RNAs; however, HRDE-1 binds germline WAGO 22G RNAs and engages the downstream Nrde pathway to direct transgenerational silencing of germline transcripts (Ashe et al., 2012; Bagijn et al., 2012; Buckley et al., 2012; Gu, S.G., et al., 2012; Luteijn et al., 2012; Shirayama et al., 2012). Loss of HRDE-1, NRDE-2, NRDE-1, or NRDE-4 results in the Mrt phenotype described above (Buckley et al., 2012), likely due to desilencing of germline targets such as transposons that pose a threat to genome integrity when active. Further testing of mutants for various upstream and downstream $21 \mathrm{U}$ RNA, 26G RNA, and WAGO 22G RNA pathway factors is necessary to determine the respective contributions of these pathways to promoting germline immortality. Curiously, the Mrt phenotype of hrde-l and nrde-2 mutants manifests only at $25^{\circ} \mathrm{C}$, whereas $n r d e-1$ and $n r d e-4$ mutants are Mrt at $25^{\circ} \mathrm{C}$ and $20^{\circ} \mathrm{C}$ (Buckley et al., 2012), suggesting the chromatin machinery of the Nrde pathway may have additional roles in germline maintenance.

Multigenerational inheritance of transcriptional silencing requires germline expression of the targeted transcript (Grishok et al., 2005). Most likely, an mRNA template must be present in the germline for RdRP to generate siRNAs capable of propagating silencing across each generation; this may account for the limited perdurance of NRDE-3-mediated somatic nuclear RNAi (Burton et al., 2011). In contrast, HRDE-1-mediated nuclear RNAi in the germline results in continued siRNA production and silencing over multiple generations that maintain H3K9 trimethylation (Ashe et al., 2012; Buckley et al., 2012; Gu, S.G., et al., 2012; Luteijn et al., 2012; Shirayama et al., 2012). Accordingly, factors such as MUT-7 and RDE-3 that are required for 22G RNA accumulation are also required for maintenance of transgenerational silencing (Luteijn et al., 2012; Shirayama et al., 2012). Screens using transgenerationally silenced exo-RNAi and 21U RNA sensor transgenes have identified a set of chromatin factors necessary for maintenance of transgenerational RNAi: the chromo domain protein HPL-2 (semiredundantly with HPL-1), Polycomb complex protein MES-3, Trithorax complex protein MES-4, SET domain SET-32, and putative H3K9 methyltransferase SET-25 (Ashe et al., 2012; Shirayama et al., 2012). These proteins may be recruited by NRDE-1 to mediate $\mathrm{H} 3 \mathrm{~K} 9$ methylation or otherwise promote a chromatin state favorable for continued siRNA biogenesis to maintain nuclear RNAi.

In the parental generation exposed to dsRNA, HRDE-1 is dispensable for germline silencing; however, HRDE-1 is required in the F1 generation for inheritance of parental germline silencing, in the F2 generation for inheritance from the $\mathrm{F} 1$, and so on, as indicated by a failure of homozygous hrde-1 mutant offspring to inherit germline transgene silencing from a $h r d e-1$ heterozygous hermaphrodite (Buckley et al., 2012). This result differs somewhat from another study, which shows that loss of HRDE-1 or MUT-7 reactivates a silenced locus only in the second generation of hrde-l homozygosity (Luteijn et al., 2012), suggesting that epigenetic silencing may be established in the parental germline.

Surprisingly, the germline nuclear RNAi pathway appears to require cytoplasmic WAGO Argonautes for initiation: during exo-RNAi targeting the oogenesis-enriched smg- 1 transcript (Reinke et al., 2004), the target site fails to become enriched for H3K9me3 marks in the MAGO mutant strain (Gu, S.G., et al., 2012). This definitively indicates that the nuclear and cytoplasmic WAGO Argonautes do not compete for siRNA cofactors. Rather, cytoplasmic WAGO Argonautes promote nuclear RNAi through an unknown mechanism. It is not yet established whether WAGO Argonautes bind 22G RNAs irreversibly or reversibly; if the latter, perhaps HRDE-1 appropriates WAGO 22G RNAs from the cytoplasmic Argonautes for nuclear silencing. NRDE-3 does not appear to be required for establishment or maintenance of transgenerational silencing (Buckley et al., 2012; Luteijn et al., 2012), nor is a sensitizing mutation in endo-RNAi factors such as ERI-1 or RRF-3 required (Gu, S.G., et al., 2012). Other WAGO Argonautes do, however, contribute variably to HRDE-1 target silencing, as some 21U RNA sensor transgenes show dependence on additional Argonautes for complete silencing (Bagijn et al., 2012; Shirayama et al., 2012).

While establishment of transgenerational silencing requires factors involved in the formation of the initial trigger, such as RDE-1 in the case of primary exo-siRNAs (Buckley et al., 2012; Gu, S.G., et al., 2012) or PRG-1 in the case of 21U RNAs (Ashe et al., 2012; Bagijn et al., 2012; Lee et al., 2012; Luteijn et al., 2012; Shirayama et al., 2012), maintenance of transgenerational silencing does not (Ashe et al., 2012; Buckley et al., 2012; Lee et al., 2012; Luteijn et al., 2012; Shirayama et al., 2012). Once established, HRDE-1-mediated nuclear silencing is stable for multiple generations and can be inherited from either the male or female germline as a dominant signal, although silencing may show a generational lag when inherited from the male (Ashe et al., 2012; Luteijn et al., 2012; Shirayama et al., 2012). This silencing in trans is mediated by a diffusible agent, namely, secondary 22G RNAs, capable of repressing homologous DNA regardless of whether the primary small RNA target site is present (Luteijn et al., 2012). These $22 \mathrm{G}$ RNAs map further upstream of the initiating $21 \mathrm{U}$ RNA target site than observed during 
early, PRG-1-dependent silencing (Bagijn et al., 2012), suggesting that transgenerational RNAi may entail additional spreading during maintenance (Luteijn et al., 2012). 22G RNAs whose expression is stimulated at transgenerationally silenced $21 \mathrm{U}$ RNA targets to maintain silencing may not correspond precisely to the species triggered by $21 \mathrm{U}$ RNA targeting, as the latter appear to be PRG-1-dependent and are lost when transgenerationally silenced loci are introduced into a prg-1 mutant background (Ashe et al., 2012).

After exposure to dsRNA, secondary siRNA accumulation and association with HRDE-1 decrease progressively across generations (Buckley et al., 2012). Transgenerational silencing initiated by endogenous small RNAs shows a similar effect, as endogenous targets of HRDE-1 silencing show progressive loss of H3K9me3 enrichment and increased expression over successive generations in a hrde-l mutant background (Buckley et al., 2012). This suggests that endogenous primary small RNA triggers may be required for periodic reinforcement of established transgenerational silencing. It is intriguing to speculate what function the nuclear RNAi pathways serve. While nuclear RNAi may simply provide a heritable signal to direct silencing of aberrant and potentially dangerous transcripts, it has also been suggested that targeting of these endogenous loci by nuclear RNAi may also fulfill a larger function in regulating chromatin dynamics.

\section{Features and targets of CSR-1 22G RNAs}

The 22G RNAs that are not bound by WAGO Argonautes are the CSR-1 22G RNAs (Figure 4). Named for their sole Argonaute effector, CSR-1, these 22G RNAs do not induce target silencing but rather associate with chromatin to promote proper organization or assembly of targets within the holocentric chromosomes of $C$. elegans (Claycomb et al., 2009). CSR-1 22G RNAs are produced in the germline by the same DRH-3- and EKL-1-containing RdRP module as WAGO 22G RNAs, but require EGO-1 and not RRF-1 for accumulation (Claycomb et al., 2009; Gu et al., 2009). Although CSR-1 22G RNAs share the features and biogenesis machinery of the secondary siRNAs engaged by WAGO Argonautes, there is no evidence that CSR-1 22G RNAs are themselves secondary siRNAs.

\section{CSR-1 associated 22G siRNA pathway}

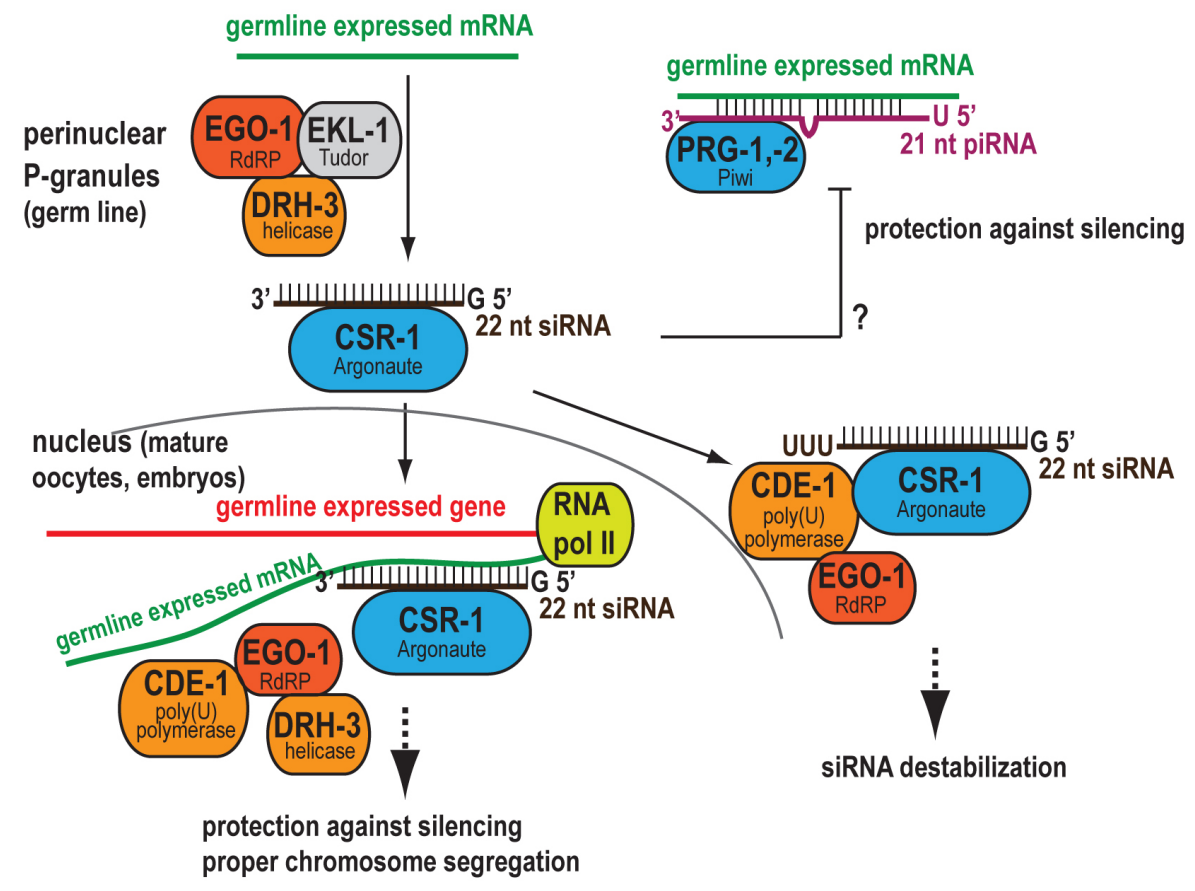

Figure 4. The CSR-1 22G siRNA pathway. In the germline P granules, the RdRP EGO-1, in complex with the helicase DRH-3 and the Tudor domain protein EKL-1, generates 22G siRNAs from over 4000 protein-coding, germline-expressed genes. These 22G siRNAs associate with the Argonaute CSR-1. CSR-1:siRNA complexes do not silence their targets, but rather localize to chromosomes to ensure proper meiotic and mitotic chromosome segregation and to protect against silencing. The poly(U)polymerase CDE-1 regulates the abundance of CSR-1 associated $22 \mathrm{G}$ siRNAs by uridylation. CSR-1 is thought to protect its targets against PRG-1 recruitment. 
CSR-1 22G RNAs largely target germline-expressed genes. Immuno-purified CSR-1 complexes are enriched for a set of $22 \mathrm{G} \mathrm{RNAs}$ that are antisense to over 4,000 protein-coding genes, whereas microRNAs, 21U RNAs, and 22G RNAs targeting repetitive sequences, pseudogenes, and intergenic or unannotated loci are depleted (Claycomb et al., 2009). Similarly, comparison of deep sequencing libraries generated from $c s r-1$ and ego- 1 mutants (Claycomb et al., 2009) with $d r h-3$ and ekl-1 mutants (Gu et al., 2009) reveals that CSR-1 and EGO-1 are required for accumulation of $22 \mathrm{G}$ RNAs antisense to protein-coding genes, many of which also require DRH-3 and EKL-1 (Claycomb et al., 2009). Most CSR-1 22G RNAs are depleted in the absence of germline (Gu et al., 2009), consistent with the germline-restricted expression pattern of EGO-1 (Smardon et al., 2000; Maine et al., 2005; Vought et al., 2005; Claycomb et al., 2009). Less than 1\% of 22G RNAs recovered in immuno-purified CSR-1 complexes target repetitive elements and pseudogenes (Claycomb et al., 2009).

\section{The CSR-1 22G RNA pathway molecular machinery}

The 22G RNA RdRP module is discussed in detail above; the following sections focus on the features and functions of EGO-1, DRH-3, and EKL-1 only as they pertain to the CSR-1 22G RNA pathway. In situ hybridization indicates that concentrations of ego-1, drh-3, ekl-1, and $c s r-1$ transcripts are highest in gonad and early embryo (She et al., 2009), in line with other transcript and protein localization data (Smardon et al., 2000; Reinke et al., 2004; Maine et al., 2005; Vought et al., 2005; Nakamura et al., 2007; Claycomb et al., 2009). DRH-3 and EKL-1 are not germline-restricted, as they also function in WAGO 22G RNA biogenesis in germline and soma (Claycomb et al., 2009; Gu et al., 2009).

\subsection{CSR-1}

The CSR-1 Argonaute clusters phylogenetically within the WAGO clade (Yigit et al., 2006), but does not bind WAGO 22G RNAs (Claycomb et al., 2009; Gu et al., 2009). Nevertheless, loss of CSR-1 results in partially defective germline and early zygotic transgene silencing (Yigit et al., 2006), although this may be due to defective localization of $\mathrm{P}$ granules in the absence of CSR-1 (Claycomb et al., 2009). CSR-1 contains an intact catalytic triad (Yigit et al., 2006). In a cell-free system, addition of single-stranded, 5'triphosphorylated 23 nt siRNA and target mRNA results in significant target cleavage that is $90 \%$ depleted in $\operatorname{csr}-1$ null mutant adult lysate; substitution of 5' monophosphorylated siRNA decreased cleavage efficiency considerably (Aoki et al., 2007). Recombinant CSR-1 is also capable of catalyzing robust cleavage in vitro when incubated with single-stranded 5' triphosphorylated, but not 5' monophosphorylated, siRNA and target (Aoki et al., 2007). Like the slicer activity of RISC in other organisms (Elbashir et al., 2001; Schwarz et al., 2004), CSR-1-mediated cleavage in cell lysate requires magnesium ions and is abolished by mutation of the two nucleotides flanking the target cleavage site (Aoki et al., 2007). Although these in vitro findings demonstrate the catalytic competence of CSR-1, no direct function for its cleavage activity has been demonstrated. The $c s r-1$ gene encodes two isoforms. Both are expressed at all developmental stages, but $c s r-1$ mRNA and protein are most enriched in young adult, gravid adult, and embryo (Claycomb et al., 2009). The larger CSR-1 protein isoform is expressed throughout larval development and is not germline restricted (Claycomb et al., 2009). CSR-1 does not require EGO-1, DRH-3, or EKL-1 for stability, nor do any of those proteins require CSR-1 or another member of the RdRP module for stability (Claycomb et al., 2009).

\section{Phenotypes associated with CSR-1 22G RNA pathway compromise}

EGO-1, DRH-3, EKL-1, and CSR-1 represent the main components of the CSR-1 22G RNA pathway (Claycomb et al., 2009). Earlier phenotypic analyses and focused or genome-wide RNAi screens have identified an overlapping set of phenotypes associated with depletion of these proteins that include embryonic lethality, larval arrest, and sterility (Qiao et al., 1995; Fraser et al., 2000; Smardon et al., 2000; Maeda et al., 2001; Zipperlen et al., 2001; Kamath et al., 2003; Simmer et al., 2003; Rual et al., 2004; Fernandez et al., 2005; Sonnichsen et al, 2005; Duchaine et al., 2006; Lehner et al., 2006; Yigit et al., 2006). These four factors were first implicated in a common pathway by their shared enhancement of $\underline{k} s r-1$ lethality (Ekl) phenotype, which describes a failure to specify excretory duct cell fate in late embryogenesis (Rocheleau et al., 2008). Transcripts whose loss produces an Ekl phenotype are expressed in maternal germline to regulate embryonic gene expression (Rocheleau et al., 2008).

\subsection{Germline phenotypes}

Mutation or RNAi-mediated depletion of ego-1, drh-3, ekl-1, or csr-1 results in common mitotic and meiotic defects leading to sterility (Qiao et al., 1995; Simmer et al., 2003; Robert et al., 2005; Duchaine et al., 2006; Yigit et al., 2006). In general, loss of these factors results in underproliferation of the germline, with nuclei of abnormal 
shape and size, (Vought et al., 2005; Maine et al., 2005; Duchaine et al., 2006; Claycomb et al., 2009; She et al., 2009). ego-1 mutants show moderately reduced germ cell number, enlargement of the transition zone with large, diffuse transition zone nuclei, reduction of the pachytene zone, and overall delayed gametogenesis (Qiao et al., 1995; Smardon et al., 2000; Vought et al., 2005). The spermatogenesis-to-oogenesis transition is also delayed, resulting in production of excess sperm (Smardon et al., 2000). Sperm produced by an ego-1 mutant are incapable of fertilization, and oocytes are small, nonfunctional, and show unpaired homologous chromosomes (Qiao et al., 1995; Smardon et al., 2000). Like ego-1 mutants, $d r h$-3, ekl-1, and csr-1 mutant germlines show protracted transition zones that include some large nuclei with diffuse chromosome morphology (Duchaine et al., 2006; She et al., 2009). These mutants also show aberrantly segregated chromosomes in diakinesis oocyte nuclei, suggesting defects in pairing, synapsis, or recombination (Nakamura et al., 2007; Claycomb et al., 2009; She et al., 2009). $d r h-3$ and $e k l-1$ mutants produce sperm with abnormally large and variably sized nuclei, indicating impaired chromatin condensation or segregation; a similar phenotype is observed in an ego-1; csr-1 double mutant (She et al., 2009). These phenotypes suggest pervasive chromosome segregation defects.

\subsection{Embryonic phenotypes}

Loss of EGO-1, DRH-3, EKL-1, or CSR-1 results in embryonic lethality (Duchaine et al., 2006; Yigit et al., 2006; Rocheleau et al., 2008). Fertilized ego-1 mutant oocytes produce eggshells, but arrest as balls of 20-50 cells without undergoing morphogenesis or gastrulation (Smardon et al., 2000). Similarly, RNAi-mediated depletion of ego-1, drh-3, ekl-1, or csr-1 results in production of embryos that show normal prophase chromosome condensation, poor metaphase alignment, chromosomal bridging at anaphase, and bisection of lagging chromosomes by the cleavage furrow at cytokinesis during every cell division; these defects cause accumulation of abnormally shaped nuclei with non-wild-type chromosomal complements and result in embryonic lethality due to arrest between gastrulation and early morphogenesis (Duchaine et al., 2006; Yigit et al., 2006; Nakamura et al., 2007; Claycomb et al., 2009). $d r h-3$ hypomorphic mutants and a $c s r-1$ mutant partially rescued by transgenic CSR-1 expression both produce dead embryos arrested at various points in embryogenesis that contain many nuclei with abnormal DNA content (Claycomb et al., 2009; Gu et al., 2009). A Him phenotype is also observed in these mutants, confirming impairment of chromosome segregation (Claycomb et al., 2009).

\section{Localization and assembly of the CSR-1 22G RNA complex}

\subsection{Germline localization}

In the germline, EGO-1, DRH-3, and CSR-1, but not EKL-1, localize to P granules and promote or maintain P granule structure and association with the nuclear periphery (Claycomb et al., 2009). As oocytes mature, EGO-1 is lost from P granules, but some DRH-3 and CSR-1 continue to associate with P granules in germ cells throughout the life cycle. DRH-3 recruits EGO-1 to the P granule, which recruits CSR-1 in an EKL-1-dependent manner. This assemblage then drives $\mathrm{P}$ granule association with the nuclear periphery, possibly by binding mRNA targets as they exit through nuclear pores; such a mechanism would be consistent with loss of $\mathrm{P}$ granule perinuclear localization in states of low transcription such as in early germ cells or oocytes (Claycomb et al., 2009). Mutation of ego-1, drh-3, ekl-1, or csr-1 disrupts perinuclear localization of P granules (Vought et al., 2005; Claycomb et al., 2009), possibly contributing to the Rde phenotypes exhibited by these mutants (Smardon et al., 2000; Kim et al., 2005; Robert et al., 2005; Duchaine et al., 2006; Yigit et al., 2006; Gu et al., 2009). These four factors may also promote normal nuclear pore structure. In wild type germlines, $\mathrm{P}$ granules are detected near nuclear pore clusters, although not every nuclear pore is associated with a P granule (Pitt et al., 2000). Pachytene chromosomes are not located adjacent to nuclear pores and may not be able to attach to the nuclear envelope in regions of high nuclear pore density (Pitt et al., 2000). Because the nuclear envelope assembles around chromatin, the chromatin may therefore influence distribution of nuclear pores (Vasu and Forbes, 2001). Loss of EGO-1 results in a patchy distribution of nuclear pore marker nucleoporin in mitotic and transition zones that might reflect abnormal chromatin dynamics (Vought et al., 2005). In sperm and oocytes, CSR-1 is detected in chromatin fractions (Chu et al., 2006), consistent with immunofluorescence data indicating that CSR-1 and EGO-1 are enriched in mature oocyte nuclei, with CSR-1 associating with diakinetic chromosomes (Claycomb et al., 2009).

\subsection{Embryo localization}

EGO-1, DRH-3, EKL-1, and CSR-1 localize to mitotic chromosomes in embryos (Claycomb et al., 2009). In embryonic cells, all four factors show cytoplasmic localization. During mitosis, however, they show nuclear enrichment beginning at prophase. As embryonic mitotic chromosomes condense, EGO-1, DRH-3, and EKL-1 become enriched along the length of each chromosome, but CSR-1 remains nuclear. At the metaphase plate, DRH-3 
and CSR-1 localize to chromosomes in a pattern similar to that of cohesins, whereas EKL-1 and, to a lesser degree, EGO-1 localize to chromosomes in a pattern like that of kinetochore proteins. EKL-1 stays associated with chromosomes during anaphase, while the others are more difficult to detect. The factors assemble on chromosomes in a hierarchical order, with DRH-3 recruiting EGO-1 and EKL-1, which in turn recruit CSR-1 (Claycomb et al., 2009). In embryos, CSR-1 pathway components are found to associate directly with chromatin in a CSR-1 22G RNA-dependent manner, supporting a direct role for CSR-1 and associated 22G RNAs in promoting chromosome segregation: CSR-1 shows enrichment at CSR-1 22G RNA target loci, but not WAGO 22G RNA target loci, by chromatin immunoprecipitation; this association is lost in a $d r h-3$ mutant (Claycomb et al., 2009). As CSR-1 22G RNA target loci are distributed relatively uniformly along chromosomes, CSR-1 associates with chromatin genome-wide to influence chromosome segregation (Claycomb et al., 2009).

\section{CSR-1 22G RNA effector function}

Although CSR-1 shows cleavage activity in cell lysate (Aoki et al., 2007), CSR-1 does not appear to silence CSR-1 22G RNA targets: these targets are expressed in germline, oocyte, and embryo and do not show changes in expression upon loss of CSR-1 or DRH-3 (Claycomb et al., 2009; Gu et al., 2009). Rather, EGO-1, DRH-3, EKL-1, and CSR-1 localize to chromosomes to promote proper organization and alignment of metaphase chromosomes and proper orientation of kinetochores to opposing spindle poles (Claycomb et al., 2009). Loss of these factors disrupts chromosome condensation and cohesion: depletion of any by RNAi results in highly disorganized condensin and cohesin protein loading onto mitotic chromosomes in embryos (Claycomb et al., 2009). Loss of these factors also disrupts kinetochore assembly. Whereas inner centromeric proteins HCP-3/CENP-A and HCP-4/CENP-C should be poleward-localized on both sides of metaphase plate, embryos depleted of CSR-1 22G RNA pathway factors show dramatically disorganized loading of these proteins on metaphase chromosomes; outer kinetochore protein KLP-7/MCAK and spindle checkpoint protein BUB-1 are also misloaded onto mitotic chromosomes (Claycomb et al., 2009). Thus, the CSR-1 22G RNA pathway directly influences chromosome organization to promote proper segregation. The $\mathrm{X}$ chromosome is depleted of genes expressed in the germline (Reinke et al., 2004) and therefore of CSR-1 22G RNA targets. Nevertheless, the X chromosome usually segregates correctly in mitosis, suggesting that other mechanisms may govern $X$ chromosome segregation (Claycomb et al., 2009).

The CSR-1 22G RNA pathway also regulates H3K9me2 distribution. H3K9me2 becomes enriched on unpaired chromosomes and high-copy extrachromosomal arrays during meiosis (Kelly et al., 2002; Bean et al., 2004). Loss of EGO-1, DRH-3, EKL-1, or CSR-1 results in reduced H3K9me2 accumulation on unpaired chromosomes such as the male $\mathrm{X}$ chromosome and ectopic H3K9me2 accumulation on paired and synapsed chromosomes (Maine et al., 2005; She et al., 2009). Whereas wild-type male pachytene nuclei show a single focus of H3K9me2 labeling, ego-1, drh-3, ekl-1, or csr-1 mutant nuclei with normal pachytene morphology lack a single strong focus of $\mathrm{H} 3 \mathrm{~K} 9 \mathrm{me} 2$ labeling, and some show multiple bright $\mathrm{H} 3 \mathrm{~K} 9 \mathrm{me} 2$ foci with higher overall levels of H3K9me2 (Maine et al., 2005; She et al., 2009). Mutant nuclei, however, may be large, morphologically abnormal, and polyploid, with diffuse chromosome morphology and multiple H3K9me2 foci and high overall H3K9me2 (She et al., 2009). This demonstrates a role for CSR-1 22G RNAs in specifying chromatin modification. However, as CSR-1 22G RNAs target protein-coding genes expressed in germline (Claycomb et al., 2009; Gu et al., 2009), it is likely that CSR-1 and associated 22G RNAs are excluding repressive H3K9me2 marks from target genomic loci rather than depositing them.

Another function of CSR-1 22G RNAs may be to define areas of germline transcription. This is consistent with a proposed role for CSR-1 22G RNAs in acting as a self-recognition system in opposition to $21 \mathrm{U}$ RNA-directed silencing of DNA perceived as non-self; targeting of transcripts by CSR-1 may therefore represent a licensing process that helps to maintain expression of bona fide germline transcripts (Ashe et al., 2012; Shirayama et al., 2012). As CSR-1 22G RNAs are generated in germline through the activity of RdRP EGO-1, CSR-1 22G RNA targets are robustly expressed in maternal germline and may therefore engage CSR-1 as nascent transcripts during gametogenesis; this may define CSR-1 chromatin domains that could perdure through embryogenesis independent of transcription (Claycomb et al., 2009). Such a mechanism would account for the prominent localization of CSR-1 to chromatin in mature oocytes (Claycomb et al., 2009). Presumably, CSR-1 establishes euchromatic genomic regions and defines the boundaries of adjacent kinetochores, potentially by mediating deposition of activating chromatin marks and HCP-3/CENP-A incorporation, respectively. In embryos, CENP-A is loaded at low density onto about half of the genome, inverse to regions transcribed in germline and early embryo, and defines centromeric regions (Gassmann et al., 2012). Pre-existing CENP-A nucleosomes are not necessary to guide recruitment of new CENP-A nucleosomes, as CENP-A is not transmitted by sperm during fertilization and is unloaded and reloaded during oogenic meiotic prophase (Gassmann et al., 2012). Germline transcription of a genomic region may exclude CENP-A incorporation in progeny, with reinforcement by zygotic transcription in early embryogenesis. Domains targeted by the CSR-1 22G RNA pathway and regions enriched for the centromeric histone variant CENP-A appear 
to be mutually exclusive (Claycomb et al., 2009); therefore, the CSR-1 22G RNA pathway may serve to transmit patterns of germline transcription to early embryo. Alternatively, H3K36 methylation may fulfill this role, as its pattern also correlates inversely with CENP-A occupancy (Rechtsteiner et al., 2010; Gassmann et al., 2012).

Finally, the CSR pathway also appears to play a critical role in maturation of replication-dependent core histone mRNAs. Processing of replication-dependent core histone pre-mRNAs requires endonucleolytic cleavage in the 3'UTR just downstream of the stem-loop that in many organisms is directed by the U7 snRNA (Marzluff et al., 2008). Nematodes, however, lack the U7 snRNA (Dávila López and Samuelsson, 2008), and CSR-1 22G RNAs may fulfill this role in its stead (Avgousti et al., 2012): a majority of histone genes (66\%) are depleted by loss of CSR-1. Immuno-purified CSR-1 complexes are enriched for $22 \mathrm{G}$ RNAs targeting all core histone genes, and EGO-1-dependent 22G RNAs map to histone pre-mRNAs at the 3' end sites of mature histone mRNAs (Avgousti et al., 2012). CSR-1 binds these 22G RNAs to target histone pre-mRNAs and either directly cleaves them or facilitates their cleavage. Loss of CSR-1 22G RNA pathway factors results in the accumulation of unprocessed histone mRNAs, with a corresponding decrease in histone production that could explain the phenotypes of these mutants (Avgousti et al., 2012). For example, histone biogenesis defects in cultured cells result in S-phase extension and cell cycle delay (Wagner et al., 2005; Ideue et al., 2009) reminiscent of the extended transition zone observed in ego-1 mutant germlines (Smardon et al., 2000). Additionally, RNAi-mediated knockdown of a single core histone gene causes chromosome segregation defects and sterility similar to the phenotypes of CSR-1 22G RNA pathway mutants (Kodama et al., 2002; Sonnichsen et al, 2005). Consistent with this explanation, overexpression of transgenic histone pre-mRNAs that do not require cleavage rescues lethality associated with $c s r-1$ or ego-l depletion by RNAi (Avgousti et al., 2012). Phenotypes similar to those shown by CSR-1 22G RNA pathway mutants also result upon depletion of the stem-loop-binding protein (SLBP), CDL-1. SLBP is required for histone mRNA processing, stabilization, and efficient translation (Marzluff et al., 2008). In the germline, CDL-1::GFP shows nuclear enrichment in developing oocytes, much like CSR-1 (Claycomb et al., 2009; Avgousti et al., 2012). RNAi-mediated knockdown of $c d l-1$ results in accumulation of unprocessed histone mRNA, severely depletes histone levels, and causes adult sterility and chromosome segregation defects in early embryo (Kodama et al., 2002; Pettitt et al., 2002; Avgousti et al., 2012). In contrast, RNAi-mediated knockdown of condensin component $s m c-4$ does not result in histone processing and accumulation defects, showing that this is not a general feature associated with compromised chromosome segregation (Avgousti et al., 2012). These results suggest that CSR-1 and CDL-1 may collaborate to promote histone maturation in oocytes, ensuring that stores of histones will be sufficient to sustain embryonic division until zygotic transcription begins. It is interesting to note that the sterility associated with compromise of the CSR-1 22G RNA pathway may be independent of the P granule defects, as loss of $c d l-1$ does not disrupt P granules (Avgousti et al., 2012). Finally, intestinal cell endoreduplication also requires high levels of histone proteins (see WormBook chapter The C. elegans intestine). Possibly related to somatic expression observed for the longer csr-1 isoform (Claycomb et al., 2009), CSR-1 protein is detected in adult intestinal nuclei (Avgousti et al., 2012). These cells show depletion of histone H2B upon RNAi knockdown of csr-1, suggesting that CSR-1 function in histone processing may not be restricted to the germline (Avgousti et al., 2012).

\section{CDE-1 regulates the CSR-1 22G RNA pathway}

The CSR-1 22G RNA pathway requires the conserved $\beta$ nucleotidyltransferase CDE-1 for uridylation of 22G RNAs to restrict their accumulation (van Wolfswinkel et al., 2009). The $c d e-1$ transcript (Reinke et al., 2004) is detected by in situ hybridization throughout the entire gonad but not in other tissues (van Wolfswinkel et al., 2009). In the male or hermaphrodite spermatogenic germline, CDE-1 localizes to bright granules in close proximity to the condensing DNA of mature sperm. In the oogenic germline, CDE-1 localization is cytoplasmic with perinuclear enrichment. Within the embryonic P lineage, CDE-1 localizes to perinuclear granules, colocalizing mainly with PGL-1; in mitotic cells, CDE-1 shows EGO-1-dependent localization to the outer edges of condensing chromosomes at prometaphase and the poleward sides of chromosomes at metaphase, with expression detectable through anaphase. CDE-1 immuno-purifies with EGO-1 in embryo extract, but CSR-1 is not required for EGO-1 accumulation. Loss of EGO-1 results in dispersed localization of CDE-1 over the whole metaphase plate and a CDE-1 halo surrounding the DNA in condensing sperm. Depletion of CSR-1 results in very faint but correct CDE-1 localization to the metaphase plate, but no association of CDE-1 granules with DNA in sperm. Loss of EGO-1 or CSR-1 does not affect CDE-1 localization to P-granules, and EGO-1 and CSR-1 show normal localization in the absence of CDE-1. CDE-1 protein is not detected in intestine (van Wolfswinkel et al., 2009).

CDE- 1 is a catalytically active nucleotidyltransferase. Immuno-purified CDE-1 complexes preferentially catalyze the addition of uridines to small RNA 3' ends in vitro (van Wolfswinkel et al., 2009), consistent with earlier findings expressing CDE-1 in Xenopus oocytes (Kwak and Wickens, 2007). Uridine tails added in vitro are short, suggesting low processivity, and can be blocked by 2'-O-methylation of the substrate small RNA 3' end (van Wolfswinkel et al., 2009). In vivo, CDE-1 uridylates a portion of CSR-1 22G RNAs. Endo-siRNAs, but not 
microRNAs, show addition of one or more untemplated 3' uridines that are lost in the absence of CDE-1 in adults; however, some endo-siRNAs in young adult still show untemplated uridines in a cde-1 mutant, suggesting redundant terminal uridylation activity at earlier stages (van Wolfswinkel et al., 2009). It is not clear whether CDE-1 uridylates $22 \mathrm{G}$ RNAs already bound by CSR-1; however, no association between the two proteins is detected in embryo extract (van Wolfswinkel et al., 2009). Uridylation by CDE-1 appears to destabilize CSR-1 22G RNAs, as those frequently found uridylated in wild type are increased upon loss of CDE-1, whereas levels of microRNAs, $21 \mathrm{U}$ RNAs, and other antisense small RNAs are not (van Wolfswinkel et al., 2009). Accordingly, immuno-purification of CSR-1 complexes from a cde-1 mutant recovers many more siRNAs, although the distribution of targets does not change (van Wolfswinkel et al., 2009). This suggests that CDE-1 acts to reduce the half-life of CSR-1 22G RNAs, ensuring that CSR-1 is only partially loaded, and, indeed, CSR-1 22G RNAs are rarer by far than WAGO 22G RNAs (Claycomb et al., 2009; Gu et al., 2009). Their modest accumulation may also explain why CSR-1 22G RNA targets are not silenced, as only the more abundantly WAGO 22G RNA-targeted loci are appreciably silenced (Claycomb et al., 2009; Gu et al., 2009). CDE-1-mediated uridylation and partial loading of CSR-1 may serve to restrict CSR-1 22G RNAs to the CSR-1 22G RNA pathway, as loss of CDE-1 results in erroneous gene silencing likely due to misloading of CSR-1 22G RNAs onto WAGO 22G RNA Argonautes (van Wolfswinkel et al., 2009). This may explain the phenotypes of $c d e-1$ mutants, which show defects in cosuppression, increased Tc1 transposon activity, and partial insensitivity to RNAi in the germline (Robert et al., 2005; van Wolfswinkel et al., 2009). In the absence of CDE-1-mediated uridylation, excess CSR-1 22G RNAs compete with WAGO 22G RNAs for Argonatute binding, compromising exo-RNAi in the germline. Similarly, Tc1-targeting siRNAs are decreased, possibly due to decreased stabilization by available WAGO Argonautes, resulting in Tc1 activation and increased dsDNA breaks and apoptosis (van Wolfswinkel et al., 2009).

Loss of CDE-1 leads to meiotic and embryonic mitotic segregation defects with a resulting Him phenotype, presumably due to erroneous targeting of CSR-1 22G RNA targets by WAGO Argonautes (van Wolfswinkel et al., 2009). Germline nuclei do not show mitotic missegregation, but diakinetic oocytes often show univalents, suggesting impaired pairing of homologous chromosomes during meiosis (van Wolfswinkel et al., 2009). cde-1 mutant embryos show metaphase plate disorganization similar to that observed in $c s r-1$ and ego-l mutant embryos, with abnormal CENP-A deposition, and occasional severe spindle defects and polar body retention (Smardon et al., 2000; Yigit et al., 2006; Claycomb et al., 2009; van Wolfswinkel et al., 2009). In analysis of cde-1 mutant hermaphrodite $\mathrm{x}$ wild-type male hybrid embryos, both parental chromosomes are affected by major segregation defects, indicating that missegregation is not limited to the parental germline and persists into embryonic development (van Wolfswinkel et al., 2009). Consistent with the collaborative but antagonistic functions of CDE-1 and CSR-1 22G RNA pathway factors, the embryonic lethality of $c d e-1$ mutants is less severe than that of $c s r-1$ and ego-1 mutants (van Wolfswinkel et al., 2009). CDE-1 also regulates processing of pre-let-7 through uridylation (Heo et al., 2008) and may act in additional roles in other small RNA pathways.

\section{21U RNA features and loci}

The following pertains to canonical or "Type 1" 21U RNAs. "Type 2" 21U RNAs are associated with transcription start sites (TSSs) of protein-coding mRNAs and other Pol II transcripts and are discussed below.

Early deep sequencing of $C$. elegans small RNAs identified a highly diverse population of $21 \mathrm{nt}$ small RNAs with a 5' uridine bias and no other common sequence features (Ruby et al., 2006). These 21U RNAs are 5' monophosphorylated (Ruby et al., 2006) and terminally methylated by HENN-1 methyltransferase (Ruby et al., 2006; Billi et al., 2012; Kamminga et al., 2012; Montgomery et al., 2012). Unlike endo-siRNAs, 21U RNAs are not generated from mRNA templates by RdRPs but rather transcribed directly from genomic loci primarily clustered in two gene-depleted regions of chromosome IV (Ruby et al., 2006; Gu, W., et al., 2012). 21U RNA loci are vastly depleted for overlap with exons. Those that do overlap show no sense or antisense bias (Ruby et al., 2006). 21U RNA loci do not show a prominent strand bias and overlap less than expected by chance (Ruby et al., 2006), consistent with their independent transcription from short precursors, (Gu, W., et al., 2012; Billi et al, 2013). Although the larger genomic clusters of 21U RNA loci show synteny across rhabditids, the 21U RNAs themselves show no sequence conservation (Ruby et al., 2006; de Wit et al., 2009). This lack of conservation suggests that evolutionary pressure has maximized the diversity of $21 \mathrm{U}$ RNA sequences, in line with their purported role as a genetic immune system capable of selectively targeting non-self transcripts (Ashe et al., 2012; Lee et al., 2012; Shirayama et al., 2012). Consistent with their free evolution, the sequence content of a 21U RNA appears to be irrelevant for expression, with the exception of the 5' uridine (Billi et al, 2013). 
21U RNAs are robustly expressed in the germline (Batista et al., 2008; Das et al., 2008) and detected in early embryo, but their levels decline across development (Stoeckius et al., 2009). A majority of 21U RNAs show differential expression in male and female germlines (Billi et al, 2013; Shi et al., 2013). These male and female germline-enriched subpopulations of 21U RNAs direct the production of nonoverlapping 22G RNAs that target distinct transcripts. Targets of male germline-enriched 21U RNAs are depleted for transcripts enriched in spermatogenesis but not oogenesis (Reinke et al., 2004), suggesting selection against evolution of male-expressed 21U RNAs that target male germline genes (Billi et al, 2013; Shi et al., 2013). Female germline-enriched 21U RNAs do not show depletion of spermatogenesis-enriched transcripts and are paradoxically enriched for oogenesis-enriched transcripts. While the significance of this is uncertain, the distinct enrichment patterns suggest that male and female germline-enriched 21U RNAs are subject to different evolutionary pressures (Billi et al, 2013; Shi et al., 2013). Female germline-enriched 21U RNAs are preferentially detected in embryos (Billi et al, 2013; Shi et al., 2013), similar to the pattern observed for ERGO-1 class 26G RNAs (Han et al., 2009).

\section{21U RNA targets}

21U RNAs target transcripts by directing Argonaute PRG-1 to imperfectly complementary sites with up to three, or possibly even four, mismatches (Bagijn et al., 2012; Lee et al., 2012). 21U RNA targets are depleted of protein-coding transcripts but not pseudogene or transposon transcripts (Bagijn et al., 2012). As 21U RNA targeting triggers local production of WAGO 22G RNAs (Das et al., 2008; Lee et al., 2012), 21U RNA targets are enriched for WAGO 22G RNA targets and depleted for CSR-1 22G RNA targets (Lee et al., 2012). As 21U RNAs are capable of triggering transgenerational silencing that persists for tens of generations in the absence of PRG-1 and additional 21U RNA trigger (Ashe et al., 2012; Luteijn et al., 2012), identification of target transcripts is challenging. Targets can be approximated as those transcripts that show robust 21U RNA-dependent 22G RNA accumulation or those that show upregulation upon loss of PRG-1, although the latter are more likely to report a specific subset of targets whose silencing is more heavily weighted toward cytoplasmic than nuclear RNAi. Possibly, the rapid evolution of $21 \mathrm{U}$ RNA sequences and the profoundly stable silencing exerted by $21 \mathrm{U}$ RNAs renders definition of a specific target set irrelevant, as many previously silenced targets have likely been eroded through genetic drift. So may be the eventual fate of sequences not protected from $21 \mathrm{U}$ RNA targeting by a self-recognition system such as CSR-1 22G RNAs are proposed to represent (Ashe et al., 2012; Shirayama et al., 2012).

\section{21U RNA biogenesis}

21U RNAs are not transcribed by RdRP complexes, nor does their biogenesis appear to require DCR-1 or any of the factors directly involved in endo-siRNA biogenesis (Batista et al., 2008; Das et al., 2008). Rather, 21U RNAs are encoded as independent Pol II transcriptional units (Gu, W., et al., 2012; Billi et al, 2013) (Figure 5). In $C$. elegans and other rhabditids, 21U RNA loci show a common upstream sequence arrangement of two motifs, the large and small motifs, that together drive the regulated expression of 21U RNAs in the germline (Ruby et al., 2006; Cecere et al., 2012; Gu, W., et al., 2012; Billi et al, 2013). In C. elegans and C. briggsae, 21U RNA loci distribution shows a pattern of enrichment at the start and end of full-length DNA transposon loci in sense and antisense orientations, respectively, suggesting that 21U RNA upstream motifs may serve as traps for triggering 21U RNA biogenesis against recent integrations (Bagijn et al., 2012). 
PRG-1/2 associated 21U piRNA pathway

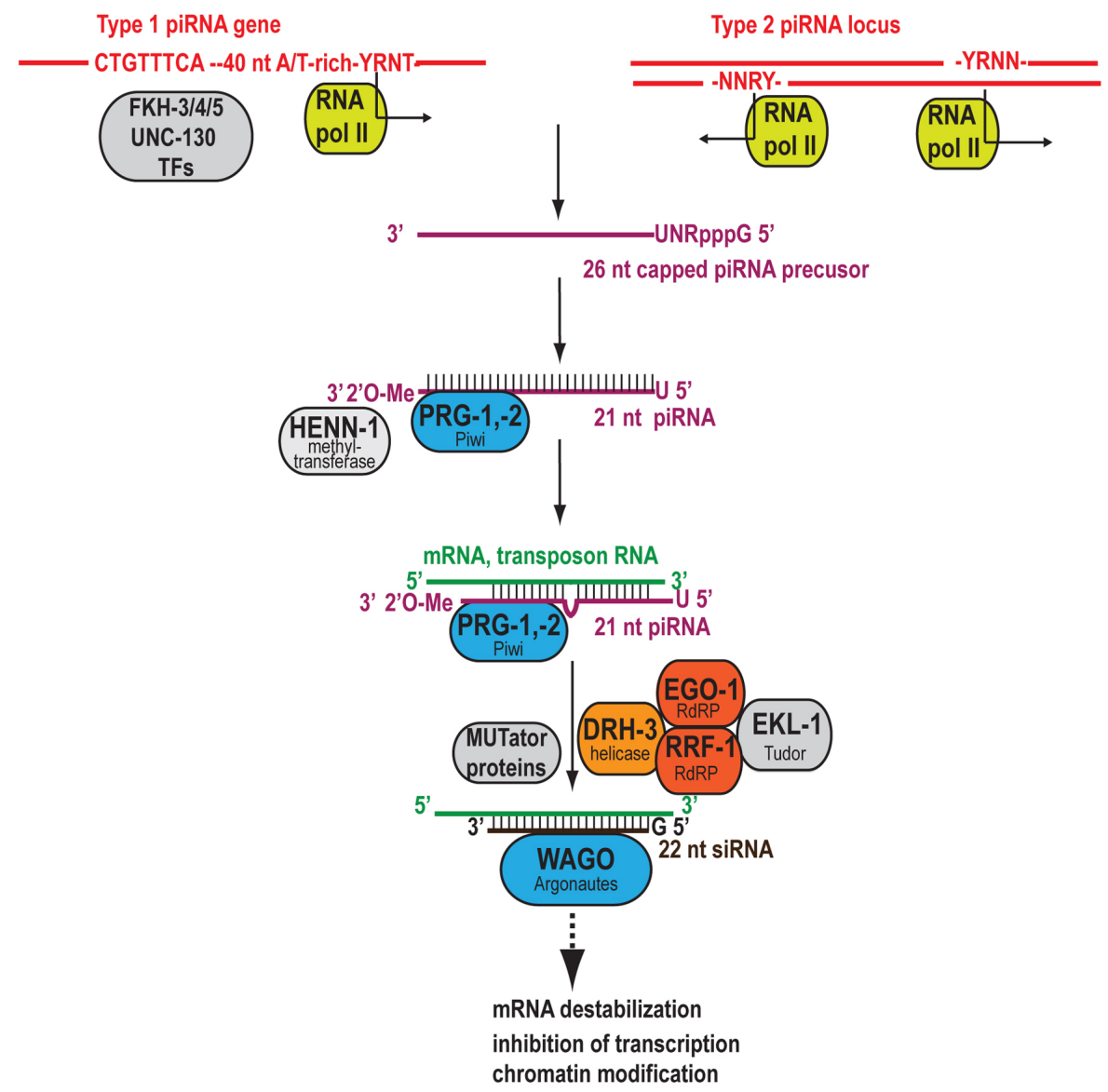

Figure 5. The 21U/piRNA pathway. piRNAs are generated from two types of loci, from piRNA genes mostly found in large clusters on chromosome IV and from protein coding genes and other Pol II transcripts. These two types of loci share a common small motif, but piRNA genes show a large motif that is bound by Forkhead transcription factors and is required to drive piRNA expression. piRNAs derived from protein coding genes map to transcription start sites and may reflect promoter proximal pausing. Both types of piRNA loci produce capped 26 nt precursors that are subsequently trimmed and 2'-O-methylated by HENN-1 to produce mature 21U RNAs. 21U RNAs associate with the Piwi proteins PRG-1/2 and trigger secondary $22 \mathrm{G}$ siRNA production.

\subsection{The 21U RNA large motif and Forkhead family transcription factors}

The large motif, occupying 60-25 bp upstream of the 21U RNA locus, consists of A/T-richness and a central 8 bp core motif of consensus sequence CTGTTTCA (Ruby et al., 2006). The A/T-richness of this region repels nucleosomes, resulting in nucleosome depletion upstream of 21U RNA locus; accordingly, the genomic 21U RNA clusters of chromosome IV show general depletion of nucleosomes (Cecere et al., 2012). The core motif is conserved in other rhabditids, but more divergent in Pristionchus pacificus; however, the spacing is conserved, suggesting mechanistic conservation (de Wit et al., 2009; Shi et al., 2013). The core motif is required for expression of the downstream 21U RNA, as its deletion or mutation results in loss of the associated 21U RNA (Cecere et al., 2012; Billi et al, 2013). A subset of Forkhead family transcription factors, including at least FKH-3, FKH-4, FKH-5, and UNC-130, binds this core motif to promote 21U RNA expression: 21U RNA upstream regions immuno-purify with UNC-130::GFP, and recombinant Forkhead proteins preferentially bind dsDNA probes containing the core motif octamer (Cecere et al., 2012). An unc-130 mutant shows 50\% depletion of individual 21U RNA species; similar depletion is observed upon partial, simultaneous RNAi knockdown of $f k h-3 / 4 / 5$ (Cecere et al., 2012). It is not yet understood whether these Forkhead proteins are semiredundantly required for expression of $21 \mathrm{U}$ RNAs or individually contribute to expression. Consistent with the core motif acting as a promoter element for independent transcription of $21 \mathrm{U}$ RNAs, the spacing between core motifs and 21U RNA loci affects 21U RNA expression, as does the sequence content. Plotting $21 \mathrm{U}$ RNA abundances by spacer length reveals a strong correlation, with spacer lengths of 37-40 nt showing the highest abundances and tailing down to either side (Ruby et al., 2006; Billi et al, 2013). The tolerance in the spacer length affords expression of multiple, genomically miniclustered $21 \mathrm{U}$ RAs in 
association with the same core motif (Kato et al., 2009; Billi et al, 2013). Core motifs that more closely match the consensus sequence, and in particular those that contain the central GTTTC, are correlated with higher abundance 21U RNAs and are more likely to be associated with 21U RNA miniclusters than solitary 21U RNAs (Batista et al., 2008; Billi et al, 2013). Miniclustered 21U RNA loci necessarily lie at different genomic distances from the core motif, and their respective abundances recapitulate the correlation between optimal spacer length and expression level (Billi et al, 2013). The sequence content of the core motif also influences germline enrichment. Core motifs whose first nucleotide is cytidine are preferentially enriched in male germline, whereas female germline-enriched 21U RNAs show no preference at this motif position and in general show core motifs with poorer consensus match (Billi et al, 2013). A simple explanation for differential germline expression of 21U RNAs according to their motifs would be selective binding by Forkhead transcription factors; however, neither UNC-130 nor FKH-3/4/5 appears to be selectively required for $21 \mathrm{U}$ RNAs expressed in male germline or showing a cytidine-intial motif (Allison Billi, Mallory Freeberg, and John Kim., unpublished data), suggesting that other factors confer germline enrichment.

\subsection{The 21U RNA small motif and precursor}

The small motif shows a YRNT consensus sequence with the thymidine situated at position 1 of the 21U RNA locus and the YR pair at 3 and 2 nt upstream, resembling the initiator element required for Pol II transcription initiation (Ruby et al., 2006; Gu, W., et al., 2012). Global profiling of endogenous capped small RNAs (csRNAs) reveals that the R position of the small motif corresponds to the first nucleotide of the $21 \mathrm{U}$ RNA precursor (Gu, W., et al., 2012). These csRNA precursors show blocked 5' ends consistent with Pol II-mediated capping and an average length of $26 \mathrm{nt}$, corresponding to the $\mathrm{m}^{7} \mathrm{GpppAmNm}$ cap- 2 structure upstream and $\sim 3 \mathrm{nt}$ downstream that are removed during 21U RNA maturation (Gu, W., et al., 2012). The abundances of csRNA precursors correlate significantly with abundances of their cognate $21 \mathrm{U}$ RNAs, supporting their status as precursors $(\mathrm{Gu}, \mathrm{W}$. , et al., 2012). A very small proportion of $21 \mathrm{U}$ RNAs show evidence of longer, $>70$ nt capped sequences (Cecere et al., 2012); however, these are unlikely to represent csRNA/21U RNA precursors, as their abundances do not correlate with abundances of cognate 21U RNAs (Gu, W., et al., 2012). The importance of the YR consensus match is indicated by the respective abundances of $21 \mathrm{U}$ RNAs encoded at loci staggered by $1 \mathrm{nt}$. Only one 21U RNA of each pair can show a YRNT motif; those that do are ten-fold more abundant than their non-YRNT sister species (Gu, W., et al., 2012).

\section{21U RNA precursor processing and Argonaute loading}

In association with an upstream large motif or at a TSS, the YR pair of the YRNT small motif appears sufficient to direct csRNA expression, whereas the thymidine is required for $21 \mathrm{U}$ RNA accumulation. The accumulation of csRNAs, but not mature 21U RNAs, associated with YNRV small motifs suggests that the 5' uridine of 21U RNAs is dispensable for transcription but plays a role in mature 21U RNA stabilization or processing (Gu, W., et al., 2012). In support of this, mutation of the thymidine to $\mathrm{G}$ or A abrogates accumulation of a cognate $21 \mathrm{G}$ or 21A species (Billi et al, 2013). Furthermore, comparison of other $C$. elegans isolates with YRNT motifs where the N2 isolate shows YRNV reveals that substitution for a genomic thymidine results in accumulation the associated 21U RNA (Gu, W., et al., 2012; Billi et al, 2013). The presence of a uridine at position 3 of the csRNA may be required for removal of the $\mathrm{m}^{7} \mathrm{GpppAmNm}$ cap-2 structure to generate the mature 21U RNA 5' end; however, recombinant PRG-1 shows preferential target cleavage in association with 5' uridine ssRNA (Bagijn et al., 2012), suggesting PRG-1 may purify candidate small RNAs processed from csRNAs by selectively binding and stabilizing those with 5' uridines. Identification of decapping machinery responsible for csRNA 5' end processing will address this question definitively. Trimming of the csRNA 3' end to achieve the mature $21 \mathrm{nt}$ length may occur after PRG-1 binding. While the vast majority of small RNA deep sequencing reads corresponding to 21U RNA loci are $21 \mathrm{nt}$ in length, a small portion show extensions, with a significant bias toward 3' extensions (Kato et al., 2009). These 3' extended reads may represent biogenesis intermediates and support the hypothesis that 3' trimming occurs after 5' processing. In silkworm, PIWI Argonaute-bound small RNAs are trimmed after Argonaute loading by a 3' to 5' exonuclease until the length is sufficiently short for anchoring of the 3' end in the Argonaute (Kawaoka et al., 2011). While the $26 \mathrm{nt}$ length of 26G RNAs is determined by DCR-1 processing, both $22 \mathrm{G}$ RNAs and $21 \mathrm{U}$ RNAs are DCR-1-independent (Aoki et al., 2007; Batista et al., 2008; Das et al., 2008; Pavelec et al., 2009). Perhaps a common exonuclease trims 22G RNAs and 21U RNAs.

\subsection{PRG-1}

PRG-1 was first identified as a factor required for germline stem cell proliferation and/or self-renewal: injection of antisense $\mathrm{prg}-1$ RNA into the germline results in significant shortening of the mitotic proliferative zone, reduced sperm production, and decreased production of offspring (Cox et al., 1998). Loss of PRG-1 results in 
decreased brood size at $20^{\circ} \mathrm{C}$ and sterility at $25^{\circ} \mathrm{C}$ (Yigit et al., 2006). This is likely due to defective sperm activation and fertilization. Spermatocytes are present at $25^{\circ} \mathrm{C}$, but mature spermatids are rare; those that do form fail to produce pseudopodia upon activation in vitro (Wang and Reinke, 2008). prg-1 mutant fertility is rescued to wild-type levels by mating to wild-type males at $20^{\circ} \mathrm{C}$, but still shows an average of half the wild-type brood size when out-crossed at $25^{\circ} \mathrm{C}$ (Wang and Reinke, 2008), suggesting compromised female fertility as well. PRG-1 is one of three PIWI clade Argonautes encoded by the C. elegans genome: ERGO-1 is highly divergent, whereas PRG-1 and PRG-2 show 91\% amino acid identity and are likely the result of a recent gene duplication (Cox et al., 1998; Yigit et al., 2006; Das et al., 2008). Despite their nearly identical sequences, only PRG-1 is required for 21U RNA accumulation (Batista et al., 2008; Das et al., 2008). Expression of prg-1 mRNA is highly germline-restricted and detected primarily in young adult and gravid adult (Batista et al., 2008; Das et al., 2008). PRG-1 protein is abundant in young adult, gravid adult, and embryo, suggesting maternal inheritance (Batista et al., 2008). Throughout the germline, PRG-1 associates consistently with P granules, with perinuclear localization in mitotic and meiotic zones, loss of expression during spermatid maturation, and dissociation from the nuclear periphery in mature oocytes (Batista et al., 2008; Wang and Reinke, 2008). Embryonic PRG-1 is also associated with P granules in the P lineage (Batista et al., 2008). PRG-1 is required for stabilizing 21U RNAs, and therefore accumulation of 21U RNAs parallels PRG-1 protein expression (Reinke et al., 2004; Batista et al., 2008; Das et al., 2008). PRG-1 binds 21U RNAs to target transcripts that are often imperfectly complementary by triggering biogenesis in trans of WAGO 22G RNAs (Das et al., 2008; Bagijn et al., 2012; Lee et al., 2012). These 21U RNA-dependent 22G RNAs can trigger transgenerational silencing by binding HRDE-1 and engaging the germline nuclear RNAi pathway (Ashe et al., 2012; Luteijn et al., 2012; Shirayama et al., 2012). Loss of PRG-1 results in target derepression due to failure to trigger dependent 22G RNAs: in particular, levels of Tc3 transposase increase and WAGO 22G RNAs targeting Tc3 decrease (Batista et al., 2008; Das et al., 2008; Gu et al., 2009). However, transcripts upregulated by loss of PRG-1 may not represent the full set of targets silenced by $21 \mathrm{U}$ RNAs, as transgenerational silencing engaged by $21 \mathrm{U}$ RNA-dependent WAGO 22G RNAs can be effectively maintained in the absence of 21U RNA trigger (Ashe et al., 2012; Lee et al., 2012; Luteijn et al., 2012; Shirayama et al., 2012). This, in addition to the fact that PRG-1-mediated targeting may not trigger WAGO 22G RNA biogenesis as effectively as other primary small RNA triggers (Bagijn et al., 2012; Lee et al., 2012), likely explains the subtle effect of 21U RNA loss upon global transcript expression (Batista et al., 2008). The catalytic triad of PRG-1 is intact, and recombinant PRG-1 is capable of cleaving target RNA when incubated with complementary 5' uridine ssRNA; cleavage is abrogated by mutating a key catalytic residue or by introducing a mismatch at the cleavage site (Bagijn et al., 2012). Nevertheless, introduction of catalytically inactive PRG-1 appears to rescue prg- 1 mutant 21U RNA accumulation, 22G RNA triggering, and target silencing, although the rescue of fertility may be imperfect (Bagijn et al., 2012; Lee et al., 2012). This suggests that, much like ERGO-1 class 26G RNAs, 21U RNAs silence targets primarily by triggering biogenesis of WAGO 22G RNAs that engage cytoplasmic and nuclear Argonautes.

\subsection{HENN-1}

HENN-1 protein and loss of function phenotypes are described in greater detail above. HENN-1 terminal methylates 21U RNAs universally (Billi et al., 2012; Kamminga et al., 2012; Montgomery et al., 2012). Although HENN-1 does not obviously localize to P granules (Billi et al., 2012) and stable interaction between PRG-1 and HENN-1 could not be detected (Kamminga et al., 2012), 21U RNA methylation presumably occurs after Argonaute loading, in analogy to ERGO-1, and 3' end trimming (Billi et al., 2012). HENN-1 is detected in a larger complex of $\sim 100 \mathrm{kD}$, suggesting that HENN-1 may act as a complex or associate with another factor, such as perhaps a trimmer exonuclease (Kamminga et al., 2012). HENN-1 is required for robust 21U RNA inheritance into embryo and perdurance in offspring (Billi et al., 2012; Montgomery et al., 2012), but loss of HENN-1 shows a lesser effect on $21 \mathrm{U}$ RNA accumulation in the adult germline (Billi et al., 2012; Kamminga et al., 2012). In contrast, ERGO-1 class 26G RNAs are quite significantly affected (Billi et al., 2012; Kamminga et al., 2012; Montgomery et al., 2012). This discrepancy does not appear to be due to differences in targeting complementarity preferentially triggering $26 \mathrm{G}$ RNA degradation, however, as the presence of a perfectly complementary 21U RNA target does not appear to stimulate 21U RNA trimming and tailing upon loss of methylation (Kamminga et al., 2012). Furthermore, both classes of 26G RNAs show roughly equivalent frequency, and 21U RNAs the lowest, of trimming and tailing among major small RNA classes in a wild-type background (Montgomery et al., 2012), suggesting that terminal methylation in C. elegans may not simply exist to oppose trimming and tailing. Nevertheless, loss of HENN-1 does result in increased levels of $20 \mathrm{nt}$ reads corresponding to 21U RNAs (Kamminga et al., 2012). The proportion of a particular 21U RNA species that is trimmed is highly correlated across different henn-1 mutant libraries, and $21 \mathrm{U}$ RNAs that show higher trimming frequencies are more likely to be depleted in henn-1 mutant libraries, indicating that 21U RNA trimming is related to greater instability and varies by species (Kamminga et al., 2012). The presence of HENN-1 appears to enhance 21U RNA-mediated silencing of a reporter transgene, but this may be an indirect 
consequence of decreased expression of factors involved in the WAGO 22G RNA pathway (Kamminga et al., 2012). Loss of HENN-1 results in extremely modest, if any, effect on levels of Tc3 transposase (Billi et al., 2012; Kamminga et al., 2012; Montgomery et al., 2012) and no increase in Tc3 transposition (Kamminga et al., 2012).

\section{TSS-associated 21U RNAs}

Mapping of csRNAs reveals that a majority are not associated with upstream core CTGTTTCA sequences; rather, many of these map bidirectionally to TSSs of protein-coding and other Pol II transcripts (Gu, W., et al., 2012). Like chromosome IV csRNAs associated with large motifs, these TSS-associated csRNAs are also processed into mature 21U RNAs when encoded by YRNT motifs (Gu, W., et al., 2012). The abundance of csRNAs at a particular promoter correlates with the abundance of longer capped reads originating from that promoter, suggesting that these csRNAs are products of Pol II initiation at active promoters and may reflect promoter-proximal pausing (Gu, W., et al., 2012). The significance of these TSS-associated 21U RNAs is as yet unknown. Much as Pol II initiation at canonical TSSs produces both csRNAs and longer pre-mRNA transcripts, a small fraction of 21U RNA loci show both csRNAs and corresponding longer reads (Cecere et al., 2012; Gu, W., et al., 2012). This suggests that promoter elements and 21U RNA upstream motifs may both promote Pol II association and transcription initiation, but 21U RNA loci generally lack sequence elements or chromatin configuration necessary to promote further transcript elongation. csRNA distribution does reveal differences in 21U RNA and canonical promoters, as bidirectional transcription is not reported to occur at 21U RNA loci. 21U RNAs are required for full fertility (Yigit et al., 2006; Batista et al., 2008; Das et al., 2008; Wang and Reinke, 2008), so it seems possible that csRNAs mapping to canonical promoters are incidental byproducts of selection for Pol II activity that produces 21U RNA precursors. However, promoter-associated csRNAs are observed in other metazoan genomes (Rasmussen and Lis, 1995; Nechaev et al., 2010), suggesting rather that 21U RNA production may originally have arisen incidental to this more ancient process of unknown significance.

\section{Future directions}

As a model organism, C. elegans rose to fame for its utility in the study of cell differentiation and organ development, yet this nematode has proven one of the richest models for study of small RNAs. The earlier challenges-developing methods to capture small RNAs of unknown properties, distinguishing the unique subclasses, identifying the factors involved in their biogenesis and effector mechanisms- have been met due to the ingenuity of pioneers in the field. Advances in sequencing technology have allowed us to catalogue the small RNAs of $C$. elegans to greater depth than ever anticipated, and new approaches continue to reveal additional complexity, such as the recently identified TSS-associated 21U RNAs found through the novel CapSeq method (Gu, W., et al., 2012). Genetic screens have also been invaluable in defining the protein factors that act in these pathways. Early screens identified suites of candidate small RNA pathway factors using complex RNAi-related phenotypes as readouts for incompletely understood underlying mechanisms. The roles of these candidates are still being elucidated, for example, chromatin factors previously implicated in transgene or transposon silencing (Kelly and Fire, 1998; Vastenhouw et al., 2003; Grishok et al., 2005; Kim et al., 2005) have only just been positioned downstream of the nuclear RNAi pathway and transgenerational silencing (Ashe et al., 2012; Shirayama et al., 2012).

In this young field of small RNAs, large, fundamental questions at every step remain unanswered. In biogenesis: How are transcripts selected for targeting by 26G RNAs? Currently, there is only speculation that secondary structure may somehow contribute to recruitment of biogenesis factors. In amplification: How does primary small RNA recognition trigger local biogenesis of secondary siRNAs? The key may lie in Mutator foci or other such physical cellular substructures that serve as amplification factories. In silencing: What are the chief post-transcriptional mechanisms by which siRNAs repress target mRNAs? The cleavage activity of many C. elegans Argonautes appears dispensable or non-conserved, contradicting the RNAi canon of Argonaute-mediated target cleavage. And particularly in broader function: What are the molecular mechanisms by which CSR-2 22G RNAs promote chromosome segregation? Why does loss of male 26G RNAs lead to defects in spermatogenesis? How does loss of the nuclear RNAi pathway compromise germline immortality? The final questions that bear upon us are those of conservation. Mammalian piRNAs show entirely unique biogenesis mechanisms, although downstream aspects of piRNA biology are shared with the $21 \mathrm{U}$ RNAs. This does not exclude the possibility that mammals express small RNAs from tiny transcriptional units like 21U RNA loci; discovery of small RNA classes and mechanisms in mammals is not yet at saturation. Although many of the pathways found in C. elegans are unlikely to be conserved in higher animals lacking the greatly expanded Argonaute family and its worm-specific clade, analogous or partially overlapping pathways may function in the germlines of other organisms. microRNAs, once 
regarded as a peculiarity of $C$. elegans genetics, have emerged as master regulators of most developmental processes. Endo-siRNAs and piRNAs appear to occupy a similar position of prominence in the C. elegans germline and embryo. It remains to be seen how this role translates in higher organisms.

\section{References}

Alder, M.N., Dames, S., Gaudet, J., and Mango, S.E. (2003). Gene silencing in Caenorhabditis elegans by transitive RNA interference. RNA 9, 25-32. Abstract Article

Ambros, V., Lee, R.C., Lavanway, A., Williams, P.T., and Jewell, D. (2003). MicroRNAs and other tiny endogenous RNAs in C. elegans. Curr. Biol. 13, 807-818. Abstract Article

Aoki, K., Moriguchi, H., Yoshioka, T., Okawa, K., and Tabara, H. (2007). In vitro analyses of the production and activity of secondary small interfering RNAs in C. elegans. EMBO J. 26, 5007-5019. Abstract

Ashe, A., Sapetschnig, A., Weick, E.M., Mitchell, J., Bagijn, M.P., Cording, A.C., Doebley, A.L., Goldstein, L.D., Lehrbach, N.J., Le Pen, J. et al. (2012). piRNAs can trigger a multigenerational epigenetic memory in the germline of C. elegans. Cell 150, 88-99. Abstract Article

Asikainen, S., Heikkinen, L., Wong, G., and Storvik, M. (2008). Functional characterization of endogenous siRNA target genes in Caenorhabditis elegans. BMC Genomics 9, 270. Abstract Article

Asikainen, S., Storvik, M., Lakso, M., and Wong, G. (2007). Whole genome microarray analysis of C. elegans rrf-3 and eri-1 mutants. FEBS Lett. 581, 5050-5054. Abstract

Avgousti, D.C., Palani, S., Sherman, Y., and Grishok, A. (2012). CSR-1 RNAi pathway positively regulates histone expression in C. elegans. EMBO J. 31, 3821-3832. Abstract Article

Bagijn, M.P., Goldstein, L.D., Sapetschnig, A., Weick, E.M., Bouasker, S., Lehrbach, N.J., Simard, M.J., and Miska, E.A. (2012). Function, targets, and evolution of Caenorhabditis elegans piRNAs. Science 337, 574-578. Abstract Article

Batista, P.J., Ruby, J.G., Claycomb, J.M., Chiang R., Fahlgren, N., Kasschau, K.D., Chaves, D.A., Gu, W., Vasale, J.J., Duan, S., et al. (2008). PRG-1 and 21U-RNAs interact to form the piRNA complex required for fertility in $C$. elegans. Mol. Cell 31, 67-78. Abstract Article

Bean, C.J., Schaner, C.E., and Kelly, W.G., (2004). Meiotic pairing and imprinted X chromatin assembly in Caenorhabditis elegans. Nature Genet. 36, 100-105. Abstract

Bernstein, E., Caudy, A.A., Hammond, S.M., and Hannon, G.J. (2001). Role for a bidentate ribonuclease in the initiation step of RNA interference. Nature 409, 363-366. Abstract Article

Billi, A.C., Alessi, A.F., Khivansara, V., Han, T., Freeberg, M., and. (2012). The Caenorhabditis elegans HEN1 ortholog, HENN-1, methylates and stabilizes select subclasses of germline small RNAs. PLoS Genet. 8, e1002617. Abstract Article

Billi, A.C., Freeberg, M.A., Day, A.M., Chun, S.Y., Khivansara, V., and Kim, J.K. (2013). A conserved upstream motif orchestrates autonomous, germline-enriched expression of Caenorhabditis elegans piRNAs. PLoS Genet. 9, e1003392. Abstract Article

Blanchard, D., Parameswaran, P., Lopez-Molina, J., Gent, J., Saynuk, J.F., and Fire, A. (2011). On the nature of in vivo requirements for $r d e-4$ in RNAi and developmental pathways in C. elegans. RNA Biol. 8, 458-467. Abstract Article

Bosher, J.M., Dufourcq, P., Sookhareea, S., and Labouesse, M. (1999). RNA interference can target pre-mRNA, consequences for gene expression in a Caenorhabditis elegans operon. Genetics 153, 1245-1256. Abstract

Buckley, B.A., Burkhart, K.B., Gu, S.G., Spracklin, G., Kershner, A., Fritz, H., Kimble, J., Fire, A., and Kenedy, S. (2012). A nuclear Argonaute promotes multigenerational epigenetic inheritance and germline immortality. Nature 489, 447-451. Abstract Article 
Burkhart, K.B., Guang, S., Buckley, B.A., Wong, L., Bochner, A.F., and Kennedy, S. (2011). A pre-mRNA-associating factor links endogenous siRNAs to chromatin regulation. PLoS Genet. 7, e1002249. Abstract Article

Burton, N.O., Burkhart, K.B., and Kennedy, S. (2011). Nuclear RNAi maintains heritable gene silencing in Caenorhabditis elegans. Proc. Natl. Acad. Sci. U. S. A. 108, 19683-19688. Abstract Article

Cecere, G., Zheng, G.X., Mansisidor, A.R., Klymko, K.E., and Grishok, A. (2012). Promoters recognized by forkhead proteins exist for individual 21U-RNAs. Mol. Cell 47, 734-745. Abstract Article

Chen, C.C., Simard, M.J., Tabara, H., Brownell, D.R., McCollough, J.A., and Mello, C.C. (2005). A member of the polymerase $\beta$ nucleotidyltransferase superfamily is required for RNA interference in $C$. elegans. Curr. Biol. 15, 378-383. Abstract Article

Chu, D.S., Liu, H., Nix, P., Wu, T.F., Ralston, E.J., Yates, J.R., 3rd, and Meyer, B.J. (2006). Sperm chromatin proteomics identifies evolutionarily conserved fertility factors. Nature 443, 101-105. Abstract Article

Claycomb, J.M., Batista, P.J., Pang, K.M., Gu, W., Vasale, J.J., van Wolfswinkel, J.C., Chaves, D.A., Shirayama, M., Mitani, S., Ketting, R.F., et al. (2009). The Argonaute CSR-1 and its 22G-RNA cofactors are required for holocentric chromosome segregation. Cell 139, 123-134. Abstract Article

Collins, J., Saari, B., and Anderson, P. (1987). Activation of a transposable element in the germ line but not the soma of Caenorhabditis elegans. Nature 328, 726-728. Abstract

Conine, C.C., Batista, P.J., Gu, W., Claycomb, J.M., Chaves, D.A., Shirayama M., and Mello, C.C. (2010). Argonautes ALG-3 and ALG-4 are required for spermatogenesis-specific 26G-RNAs and thermotolerant sperm in Caenorhabditis elegans. Proc. Natl. Acad. Sci. U. S. A. 107, 3588-3593. Abstract Article

Corrêa, R.L., Steiner, F.A., Berezikov, E., Ketting, R.F. (2010). MicroRNA-directed siRNA biogenesis in Caenorhabditis elegans. PLoS Genet. 6, e1000903. Abstract Article

Cox, D.N., Chao, A., Baker, J., Chang, L., Qiao, D., and Lin, H. (1998). A novel class of evolutionarily conserved genes defined by piwi are essential for stem cell self-renewal. Genes Dev. 12, 3715-3727. Abstract Article

Das, P.P., Bagijn, M.P., Goldstein, L.D., Woolford, J.R., Lehrbach, N.J., Sapetschnig, A., Buhecha, H.R., Howe, K.L., Startk, R., Matthews, N., et al. (2008). Piwi and piRNAs act upstream of an endogenous siRNA pathway to suppress Tc3 transposon mobility in the Caenorhabditis elegans germline. Mol. Cell 31, 79-90. Abstract Article

Dávila López, M., and Samuelsson, T. (2008). Early evolution of histone mRNA 3' end processing. RNA 14, 1-10. Abstract Article

de Wit, E., Linsen, S.E., Cuppen, E., and Berezikov, E. (2009). Repertoire and evolution of miRNA genes in four divergent nematode species. Genome Res. 19, 2064-2074. Abstract Article

Duchaine, T.F., Wohlschlegel, J.A., Kennedy, S., Bei, Y., Conte, D. Jr., Pang, K., Brownell, D.R., Harding, S., Mitani, S., Ruvkun, G., et al. (2006). Functional proteomics reveals the biochemical niche of C. elegans DCR-1 in multiple small-RNA-mediated pathways. Cell 124, 343-354. Abstract Article

Elbashir, S.M., Lendeckel, W., and Tuschl, T. (2001). RNA interference is mediated by 21- and 22-nucleotide RNAs. Genes Dev. 15, 188-200. Abstract Article

Emmons, S.W., and Yesner, L. (1984). High-frequency excision of transposable element Tc 1 in the nematode Caenorhabditis elegans is limited to somatic cells. Cell 36, 599-605. Abstract Article

Fernandez, A.G., Gunsalus, K.C., Huang, J., Chuang, L.S., Ying, N., Liang, H.L., Tang, C., Schetter, A.J., Zegar, C., Rual, J.F. et al. (2005). New genes with roles in the C. elegans embryo revealed using RNAi of ovary-enriched ORFeome clones. Genome Res. 15, 250-259. Abstract Article 
Fire, A., Albertson, D., Harrison, S.W., and Moerman, D.G. (1991). Production of antisense RNA leads to effective and specific inhibition of gene expression in C. elegans muscle. Development 113, 503-514. Abstract

Fire, A., Xu, S., Montgomery, M.K., Kostas, S.A., Driver, S.E., and Mello, C.C. (1998). Potent and specific genetic interference by double-stranded RNA in Caenorhabditis elegans. Nature 391, 806-811. Abstract Article

Fischer, S.E. (2010). Small RNA-mediated gene silencing pathways in C. elegans. Int. J. Biochem. Cell Biol. 42, 1306-1315. Abstract

Fischer, S.E., Butler, M.D., Pan, Q., and Ruvkun, G. (2008). Trans-splicing in C. elegans generates the negative RNAi regulator ERI-6/7. Nature 455, 491-496. Abstract Article

Fischer, S.E., Montgomery, T.A., Zhang, C., Fahlgren, N., Breen, P.C., Hwang, A., Sullivan, C.M., Carrington, J.C., and Ruvkun, G. (2011). The ERI-6/7 helicase acts at the first stage of an siRNA amplification pathway that targets recent gene duplications. PLoS Genet. 7, e1002369. Abstract Article

Fraser, A.G., Kamath, R.S., Zipperlen, P., Martinez-Campos, M., Sohrmann, M., and Ahringer, J. (2000). Functional genomic analysis of $C$. elegans chromosome I by systematic RNA interference. Nature 408, 325-330. Abstract Article

Gabel, H.W., and Ruvkun, G. (2008). The exonuclease ERI-1 has a conserved dual role in 5.8S rRNA processing and RNAi. Nat. Struct. Mol. Biol. 15, 531-533. Abstract Article

Gassmann, R., Rechtsteiner, A., Yuen, K.W., Muroyama, A., Egelhofer, T., Gaydos, L., Barron, F., Maddox, P., Essex, A., Monen, J. et al. (2012). An inverse relationship to germline transcription defines centromeric chromatin in C. elegans. Nature 484, 534-537. Abstract Article

Gent, J.I., Lamm, A.T., Pavelec, D.M., Maniar, J.M., Parameswaran, P., Tao, L., Kennedy, S., and Fire, A.Z. (2010). Distinct phases of siRNA synthesis in an endogenous RNAi pathway in C. elegans soma. Mol. Cell 37, 679-689. Abstract Article

Gent, J.I., Schvarzstein, M., Villeneuve, A.M., Gu, S.G., Jantsch, V., Fire, A.Z., and Baudrimont, A. (2009). A Caenorhabditis elegans RNA-directed RNA polymerase in sperm development and endogenous RNA interference. Genetics 183, 1297-1314. Abstract Article

Grishok, A., Pasquinelli, A.E., Conte, D., Li, N., Parrish, S., Ha, I., Baillie, D.L., Fire, A., Ruvkun, G., and Mello, C.C. (2001). Genes and mechanisms related to RNA interference regulate expression of the small temporal RNAs that control C. elegans developmental timing. Cell 106, 23-34. Abstract

Grishok, A., Sinskey, J.L., and Sharp, P.A. (2005). Transcriptional silencing of a transgene by RNAi in the soma of C. elegans. Genes Dev. 19, 683-696. Abstract Article

Grishok, A., Tabara, H., and Mello, C.C. (2000). Genetic requirements for inheritance of RNAi in C. elegans. Science 287, 2494-2497. Abstract Article

Gu, S.G., Pak, J., Guang, S., Maniar, J.M., Kennedy, S., and Fire, A. (2012). Amplification of siRNA in Caenorhabditis elegans generates a transgenerational sequence-targeted histone $\mathrm{H} 3$ lysine 9 methylation footprint. Nature Genet. 44, 157-164. Abstract Article

Gu, W., Lee, H.C., Chaves, D., Youngman, E.M., Pazour, G.J., Conte, D., Jr., and Mello, C.C. (2012). CapSeq and CIP-TAP identify Pol II start sites and reveal capped small RNAs as C. elegans piRNA precursors. Cell 151, 1488-1500. Abstract Article

Gu, W., Shirayama, M., Conte, D., Jr., Vasale, J., Batista, P.J., Claycomb, J.M., Moresco, J.J., Youngman, E.M., Keys, J., Stoltz, M.J., et al. (2009). Distinct argonaute-mediated 22G-RNA pathways direct genome surveillance in the C. elegans germline. Mol. Cell 36, 231-244. Abstract Article

Guang, S., Bochner, A.F., Burkhart, K.B., Burton, N., Pavelec, D.M., and Kennedy, S. (2010). Small regulatory RNAs inhibit RNA polymerase II during the elongation phase of transcription. Nature 465, 1097-1101. Abstract Article 
Guang, S., Bochner, A.F., Pavelec, D.M., Burkhart, K.B., Harding, S., Lachowiec, J., and Kennedy, S. (2008). An Argonaute transports siRNAs from the cytoplasm to the nucleus. Science 321, 537-541. Abstract Article

Han, T., Manoharan, A.P., Harkins, T.T., Bouffard, P., Fitzpatrick, C., Fitzpatrick, C., Chu, D.S., Thierry-Mieg, D., Thierry-Mieg, J., and Kim, J.K. (2009). 26G endo-siRNAs regulate spermatogenic and zygotic gene expression in Caenorhabditis elegans. Proc. Natl. Acad. Sci. U. S. A. 106, 18674-18679. Abstract Article

Han, W., Sundaram, P., Kenjale, H., Grantham, J., and Timmons, L. (2008). The Caenorhabditis elegans rsd-2 and rsd-6 genes are required for chromosome functions during exposure to unfavorable environments. Genetics 178 , 1875-1893. Abstract Article

Heo, I., Joo, C., Cho, J., Ha, M., Han, J., and Kim, V.N. (2008). Lin28 mediates the terminal uridylation of let-7 precursor MicroRNA. Mol. Cell 32, 276-284. Abstract Article

Hunt-Newbury, R., Viveiros, R., Johnsen, R., Mah, A., Anastas, D., Fang, L., Halfnight, E., Lee, D., Lin, J., Lorch, A. et al. (2007). High-throughput in vivo analysis of gene expression in Caenorhabditis elegans. PLoS Biol. 5, e237. Abstract Article

Ideue, T. Hino, K., Kitao, S., Yokoi, T., and Hirose, T. (2009). Efficient oligonucleotide-mediated degradation of nuclear noncoding RNAs in mammalian cultured cells. RNA 15, 1578-1587. Abstract Article

Kamath, R.S., Fraser, A.G., Dong, Y., Poulin, G., Durbin, R., Gotta, M., Kanapin, A., Le Bot, N., Moreno, S., Sohrmann, M. et al. (2003). Systematic functional analysis of the Caenorhabditis elegans genome using RNAi. Nature 421, 231-237. Abstract Article

Kamminga, L.M., van Wolfswinkel, J.C., Luteijn, M.J., Kaaij, L.J., Bagijn, M.P., Sapetschnig, W., Miska,E.A., Berezikov, E., and Ketting, R.F. (2012). Differential impact of the HEN1 homolog HENN-1 on 21U and 26G RNAs in the germline of Caenorhabditis elegans. PLoS Genet. 8, e1002702. Abstract Article

Kato, M., de Lencastre, A., Pincus, Z., and Slack, F.J. (2009). Dynamic expression of small non-coding RNAs, including novel microRNAs and piRNAs/21U-RNAs, during Caenorhabditis elegans development. Genome Biol. 10, R54. Abstract Article

Kawaoka, S., Izumi, N., Katsuma, S., and Tomari, Y. (2011). 3' end formation of PIWI-interacting RNAs in vitro. Mol. Cell 43, 1015-1022. Abstract Article

Kelly, W.G., and Fire., A. (1998). Chromatin silencing and the maintenance of a functional germline in Caenorhabditis elegans. Development 125, 2451-2456. Abstract

Kelly, W.G., Schaner, C.E., Dernburg, A.F., Lee, M.H., Kim, S.K., Villeneuve, A.M., and Reinke, V. (2002). $\mathrm{X}$-chromosome silencing in the germline of C. elegans. Development 129, 479-492. Abstract

Kennedy, S., Wang, D., and Ruvkun, G. (2004). A conserved siRNA-degrading RNase negatively regulates RNA interference in C. elegans. Nature 427, 645-649. Abstract Article

Ketting, R.F., and Plasterk, R.H. (2000). A genetic link between co-suppression and RNA interference in C. elegans. Nature 404, 296-298. Abstract Article

Ketting, R.F., Fischer, S.E., Bernstein, E., Sijen, T., Hannon, G.J., and Plasterk, R.H. (2001). Dicer functions in RNA interference and in synthesis of small RNA involved in developmental timing in C. elegans. Genes Dev. 15, 2654-2659. Abstract Article

Ketting, R.F., Haverkamp, T.H., van Luenen, H.G., and Plasterk, R.H. (1999). mut-7 of C. elegans, required for transposon silencing and RNA interference, is a homolog of Werner syndrome helicase and RNaseD. Cell 99, 133-141. Abstract Article

Kim, J.K., Gabel, H.W., Kamath, R.S., Tewari, M., Pasquinelli, A., Rual, J.F., Kennedy, S., Dybbs, M., Bertin, N., Kaplan, J.M. et al. (2005). Functional genomic analysis of RNA interference in C. elegans. Science 308, 1164-1167. Abstract Article 
Knight, S.W., and Bass, B.L. (2001). A role for the RNase III enzyme DCR-1 in RNA interference and germ line development in Caenorhabditis elegans. Science 293, 2269-2271. Abstract Article

Kodama, Y., Rothman, J.H., Sugimoto, A., and Yamamoto, M. (2002). The stem-loop binding protein CDL-1 is required for chromosome condensation, progression of cell death and morphogenesis in Caenorhabditis elegans. Development 129, 187-196. Abstract

Kwak, J.E., and Wickens, M. (2007). A family of poly(U) polymerases. RNA 13, 860-867. Abstract Article

Lau, N.C., Lim, L.P., Weinstein, E.G., and Bartel, D.P. (2001), An abundant class of tiny RNAs with probable regulatory roles in Caenorhabditis elegans. Science 294, 858-862. Abstract Article

Lee, H.C., Gu, W., Shirayama, M., Youngman, E., Conte, D., Jr., and Mello, C.C. (2012). C. elegans piRNAs mediate the genome-wide surveillance of germline transcripts. Cell 150, 78-87. Abstract Article

Lee, R., Feinbaum, R., and Ambros, V. (1993). The C. elegans heterochronic gene lin-4 encodes small RNAs with antisense complementarity to lin-14. Cell 75, 843-854. Abstract Article

Lee, R.C., and Ambros, V. (2001). An extensive class of small RNAs in Caenorhabditis elegans. Science 294, 862-864. Abstract Article

Lee, R.C., Hammell, C.M., and Ambros, V. (2006). Interacting endogenous and exogenous RNAi pathways in Caenorhabditis elegans. RNA 12, 589-597. Abstract Article

Lehner, B., Crombie, C., Tischler, J., Fortunato, A., and Fraser, A.G. (2006). Systematic mapping of genetic interactions in Caenorhabditis elegans identifies common modifiers of diverse signaling pathways. Nature Genet. 38, 896-903. Abstract Article

Lim, L.P., Lau, N.C., Weinstein, E.G., Abdelhakim, A., Yekta, S., Rhoades, M.W., Burge, C.B., and Bartel, D.P. (2003). The microRNAs of Caenorhabditis elegans. Genes Dev. 17, 991-1008. Abstract Article

Luteijn, M.J., van Bergeijk, P., Kaaij, L.J., Almeida, M.V., Roovers, E.F., Berezikov, E. and Ketting, R.F. (2012). Extremely stable Piwi-induced gene silencing in Caenorhabditis elegans. EMBO J. 31, 3422-3430. Abstract Article

Maeda, I., Kohara, Y., Yamamoto, M., and Sugimoto, A. (2001). Large-scale analysis of gene function in Caenorhabditis elegans by high-throughput RNAi. Curr. Biol. 11, 171-176. Abstract Article

Maine, E.M., Hauth, J., Ratliff, T., Vought, V.E., She, X., and Kelly, W.G. (2005). EGO-1, a putative RNA-dependent RNA polymerase, is required for heterochromatin assembly on unpaired DNA during C. elegans meiosis. Curr. Biol. 15, 1972-1978. Abstract Article

Makeyev, E.V., and Bamford, D.H. (2002). Cellular RNA-dependent RNA polymerase involved in posttranscriptional gene silencing has two distinct activity modes. Mol. Cell 10, 1417-1427. Abstract Article

Mangone, M., Manoharan, A.P., Thierry-Mieg, D., Thierry-Mieg, J., Han, T., Mackowiak, S.D., Mis, E., Zegar, C., Gutwein, M.R., Khivansara, V. et al. (2010). The landscape of C. elegans 3'UTRs. Science 329, 432-435. Abstract Article

Marzluff, W.F., Wagner, E.J., and Duronio, R.J. (2008). Metabolism and regulation of canonical histone mRNAs, life without a poly(A) tail. Nat. Rev. Genet. 9, 843-854. Abstract Article

McGhee, J.D. The C. elegans intestine (March 27, 2007), WormBook, ed. The C. elegans Research Community, WormBook, doi/10.1895/wormbook.1.133.1, http://www.wormbook.org.

Montgomery, M.K., and Fire., A. (1998). Double-stranded RNA as a mediator in sequence-specific genetic silencing and co-suppression. Trends Genet. 14, 255-258. Abstract Article

Montgomery, M.K., Xu, S., and Fire., A. (1998). RNA as a target of double-stranded RNA-mediated genetic interference in Caenorhabditis elegans. Proc. Natl. Acad. Sci. U. S. A. 95, 15502-15507. Abstract Article 
Montgomery, T.A., Rim, Y.S., Zhang, C., Dowen, R.H., Phillips, C.M., Fischer, S.E., and Ruvkun, G. (2012). PIWI associated siRNAs and piRNAs specifically require the Caenorhabditis elegans HEN1 ortholog henn-1. PLoS Genet. 8, e1002616. Abstract Article

Moore, M.J., and Proudfoot, N.J. (2009). Pre-mRNA processing reaches back to transcription and ahead to translation. Cell 136, 688-700. Abstract Article

Nakamura, M., Ando, R., Nakazawa, T., Yudazono, T., Tsutsumi, N., Hatanaka, N., Ohgake, T., Hanaoka, F., and Eki, T. (2007). Dicer-related $d r h-3$ gene functions in germ-line development by maintenance of chromosomal integrity in Caenorhabditis elegans. Genes Cells 12, 997-1010. Abstract Article

Nechaev, S., Fargo, D.C., dos Santos, G., Liu, L., Gao, Y., and Adelman, K. (2010). Global analysis of short RNAs reveals widespread promoter-proximal stalling and arrest of Pol II in Drosophila. Science 327, 335-338. Abstract Article

Pak, J., and Fire, A. (2007). Distinct populations of primary and secondary effectors during RNAi in C. elegans. Science 315, 241-244. Abstract Article

Park, W., Li, J., Song, R., Messing, J., and Chen, X. (2002). CARPEL FACTORY, a Dicer homolog, and HEN1, a novel protein, act in microRNA metabolism in Arabidopsis thaliana. Curr. Biol. 12, 1484-1495. Abstract Article

Parker, G.S., Eckert, D.M., and Bass, B.L. (2006). RDE-4 preferentially binds long dsRNA and its dimerization is necessary for cleavage of dsRNA to siRNA. RNA 12, 807-818. Abstract Article

Parker, G.S., Maity, T.S., and Bass, B.L. (2008). dsRNA binding properties of RDE-4 and TRBP reflect their distinct roles in RNAi. J. Mol. Biol. 384, 967-979. Abstract Article

Parrish, S., and Fire, A. (2001). Distinct roles for RDE-1 and RDE-4 during RNA interference in Caenorhabditis elegans. RNA 7, 1397-1402. Abstract

Pavelec, D.M., Lachowiec, J., Duchaine, T.F., Smith, H.E., and Kennedy, S. (2009). Requirement for the ERI/DICER complex in endogenous RNA interference and sperm development in Caenorhabditis elegans. Genetics 183, 1283-1295. Abstract Article

Pettitt, J., Crombie, C., Schumperli, D., Muller, B. (2002). The Caenorhabditis elegans histone hairpin-binding protein is required for core histone gene expression and is essential for embryonic and postembryonic cell division. J. Cell Sci. 115, 857-866. Abstract

Phillips, C.M., Montgomery, T.A., Breen, P.C., and Ruvkun, G. (2012). MUT-16 promotes formation of perinuclear mutator foci required for RNA silencing in the C. elegans germline. Genes Dev. 26, 1433-1444. Abstract Article

Pitt, J.N., Schisa, J.A., and Priess, J.R. (2000). P granules in the germ cells of Caenorhabditis elegans adults are associated with clusters of nuclear pores and contain RNA. Dev. Biol. 219, 315-333. Abstract Article

Qiao, L., Lissemore, J.L., Shu, P., Smardon, A., Gelber, M.B., and Maine E.M. (1995). Enhancers of $g l p-1$, a gene required for cell-signaling in Caenorhabditis elegans, define a set of genes required for germline development. Genetics 141, 551-569. Abstract

Rasmussen, E.B., and Lis, J.T. (1995). Short transcripts of the ternary complex provide insight into RNA polymerase II elongational pausing. J. Mol. Biol. 252, 522-535. Abstract

Rechtsteiner, A., Ercan, S., Takasaki, T., Phippen, T.M., Egelhofer, T.A., Wang, W., Kimura, H., Lieb, J.D., and Strome, S. (2010). The histone H3K36 methyltransferase MES-4 acts epigenetically to transmit the memory of germline gene expression to progeny. PLoS Genet. 6, e1001091. Abstract Article

Reinhart, B.J., Slack, F.J., Basson, M., Pasquinelli, A.E., Bettinger, J.C., Rougvie, A.E., Horvitz, H.R., and Ruvkun, G. (2000). The 21-nucleotide let-7 RNA regulates developmental timing in Caenorhabditis elegans. Nature 403, 901-906. Abstract Article 
Reinke, V., Gil, I.S., Ward, S., and Kazmer, K. (2004). Genome-wide germline-enriched and sex-biased expression profiles in Caenorhabditis elegans. Development 131,311-323. Abstract Article

Robert, V.J., Sijen, T., van Wolfswinkel, J., and Plasterk, R.H. (2005). Chromatin and RNAi factors protect the $C$. elegans germline against repetitive sequences. Genes Dev 19, 782-787. Abstract Article

Rocheleau, C.E., Cullison, K., Huang, K., Bernstein, Y., Spilker, A.C., and Sundaram, M.V. (2008). The Caenorhabditis elegans ekl (enhancer of ksr-1 lethality). genes include putative components of a germline small RNA pathway. Genetics 178, 1431-1443. Abstract Article

Rual, J.F., Ceron, J., Koreth, J., Hao, T., Nicot, A.S., Hirozane-Kishikawa, T., Vandenhaute, J., Orkin, S.H., Hill, D.E., van den Heuvel, S., and Vidal, M. (2004). Toward improving Caenorhabditis elegans phenome mapping with an ORFeome-based RNAi library. Genome Res. 14, 2162-2168. Abstract Article

Ruby, J.G., Jan, C., Player, C., Axtell, M.J., Lee, W., Nusbaum, C., Ge, H., and Bartel, D.P. (2006). Large-scale sequencing reveals 21U-RNAs and additional microRNAs and endogenous siRNAs in C. elegans. Cell 127, 1193-1207. Abstract Article

Schrimpf, S.P., Weiss, M., Reiter, L., Ahrens, C.H., Jovanovic, M., Malmstrom, J., Brunner, E., Mohanty, S., Lercher, M.J., Hunziker, P.E., et al. (2009). Comparative functional analysis of the Caenorhabditis elegans and Drosophila melanogaster proteomes. PLoS Biol. 7, e48. Abstract Article

Schwarz, D.S., Tomari, Y., and Zamore, P.D. (2004). The RNA-induced silencing complex is a Mg2+-dependent endonuclease. Curr. Biol. 14, 787-791. Abstract Article

She, X., Xu, X., Fedotov, A., Kelly, W.G., and Maine, E.M. (2009). Regulation of heterochromatin assembly on unpaired chromosomes during Caenorhabditis elegans meiosis by components of a small RNA-mediated pathway. PLoS Genet. 5, e1000624. Abstract Article

Sheth, U., Pitt, J., Dennis, S., and Priess, J.R. (2010). Perinuclear P granules are the principal sites of mRNA export in adult C. elegans germ cells. Development 137, 1305-1314. Abstract Article

Shi, Z., Montgomery, T.A., Qi, Y., and Ruvkun, G. (2013). High-throughput sequencing reveals extraordinary fluidity of miRNA, piRNA, and siRNA pathways in nematodes. Genome Res. 23, 497-508. Abstract Article

Shirayama, M., Seth, M., Lee, H.C., Gu, W., Ishidate, T., Conte, D. Jr., and Mello, C.C. (2012). piRNAs initiate an epigenetic memory of nonself RNA in the C. elegans germline. Cell 150, 65-77. Abstract Article

Sijen, T., Fleenor, J., Simmer, F., Thijssen, K.L., Parrish, S., Timmons, L., Plasterk, R.H., and Fire, A. (2001). On the role of RNA amplification in dsRNA-triggered gene silencing. Cell 107, 465-476. Abstract Article

Sijen, T., Steiner, F.A., Thijssen, K.L., and Plasterk, R.H. (2007). Secondary siRNAs result from unprimed RNA synthesis and form a distinct class. Science 315, 244-247. Abstract Article

Simmer, F., Moorman, C., van der Linden, A.M., Kuijk, E., van den Berghe, P.V., Kamath, R.S., Fraser, A.g., Ahringer, J., and Plasterk, R.H. (2003). Genome-wide RNAi of $C$. elegans using the hypersensitive rrf-3 strain reveals novel gene functions. PLoS Biol. 1, e12. Abstract Article

Simmer, F., Tijsterman, M., Parrish, S., Koushika, S.P., Nonet, M.L., Fire, A., Ahringer, J., and Plasterk, R.H. (2002). Loss of the putative RNA-directed RNA polymerase RRF-3 makes C. elegans hypersensitive to RNAi. Curr. Biol. 12, 1317-1319. Abstract Article

Smardon, A., Spoerke, J.M., Stacey, S.C., Klein, M.E., Mackin, N., and Maine, E.M. (2000). EGO-1 is related to RNA-directed RNA polymerase and functions in germ-line development and RNA interference in C. elegans. Curr. Biol. 10, 169-178. Abstract

Sonnichsen, B., Koski, L.B., Walsh, A., Marschall, P., Neumann, B., Brehm, M., Alleaume, A.M., Artelt, J., Bettencourt, P., Cassin, E. et al. (2005). Full-genome RNAi profiling of early embryogenesis in Caenorhabditis elegans. Nature 434, 462-469. Abstract Article 
Steiner, F.A., Hoogstrate, S.W., Okihara, K.L., Thijssen, K.L., Ketting, R.F., Ketting, R.F., Plasterk, R.H., and Sijen, T. (2007). Structural features of small RNA precursors determine Argonaute loading in Caenorhabditis elegans. Nat. Struct. Mol. Biol.14, 927-933. Abstract Article

Steiner, F.A., Okihara, K.L., Hoogstrate, S.W., Sijen, T., and Ketting, R.F. (2009). RDE-1 slicer activity is required only for passenger-strand cleavage during RNAi in Caenorhabditis elegans. Nat. Struct. Mol. Biol.16, 207-211. Abstract Article

Stoeckius, M., Maaskola, J., Colombo, T., Rahn, H.P., Friedlander, M.R., Li, N., Chen, W., Piano, F., and Rajewsky, N. (2009). Large-scale sorting of C. elegans embryos reveals the dynamics of small RNA expression. Nat. Methods 6, 745-751. Abstract Article

Sundaram, P., Echalier, B., Han, W., Hull, D., and Timmons, L. (2006). ATP-binding cassette transporters are required for efficient RNA interference in Caenorhabditis elegans. Mol. Biol. Cell 17, 3678-3688. Abstract Article

Tabara, H., Sarkissian, M., Kelly, W.G., Fleenor, J., Grishok, A., Timmons, L., Fire, A., and Mello, C.C. (1999). The $r d e-1$ gene, RNA interference, and transposon silencing in C. elegans. Cell 99, 123-132. Abstract Article

Tabara, H., Yigit, E., Siomi, H., and Mello, C.C. (2002). The dsRNA binding protein RDE-4 interacts with RDE-1, DCR-1, and a DExH-box helicase to direct RNAi in C. elegans. Cell 109, 861-871. Abstract Article

Thivierge, C., Makil, N., Flamand, M., Vasale, J.J., Mello, C.C., Wohlschlegel, J., Conte, D. Jr., and Duchaine, T.F. (2012). Tudor domain ERI-5 tethers an RNA-dependent RNA polymerase to DCR-1 to potentiate endo-RNAi. Nat. Struct. Mol. Biol. 19, 90-97. Abstract Article

Tijsterman, M., Ketting, R.F., Okihara, K.L., Sijen, T., and Plasterk, R.H. (2002a). RNA helicase MUT-14-dependent gene silencing triggered in C. elegans by short antisense RNAs. Science 295, 694-697. Abstract Article

Tijsterman, M., May, R.C., Simmer, F., Okihara, K.L. and Plasterk, R.H. (2004). Genes required for systemic RNA interference in Caenorhabditis elegans. Curr. Biol. 14, 111-116. Abstract Article

Tijsterman, M., Okihara, K.L., Thijssen, K., and Plasterk, R.H. (2002b). PPW-1, a PAZ/PIWI protein required for efficient germline RNAi, is defective in a natural isolate of C. elegans. Curr. Biol. 12, 1535-1540. Abstract Article

Tops, B.B., Tabara, H., Sijen, T., Simmer, F., Mello, C.C., Plasterk, R.H., and Ketting, R.F. (2005). RDE-2 interacts with MUT-7 to mediate RNA interference in Caenorhabditis elegans. Nucleic Acids Res. 33, 347-355. Abstract Article

van Wolfswinkel, J.C., Claycomb, J.M., Batista, P.J., Mello, C.C., Berezikov, E., and Ketting, R.F. (2009). CDE-1 affects chromosome segregation through uridylation of CSR-1-bound siRNAs. Cell 139, 135-148. Abstract Article

Vasale, J.J., Gu, W., Thivierge, C., Batista, P.J., Claycomb, J.M., Youngman, E.M., Duchaine, T.F., Mello, C.C., and Conte, D. Jr. (2010). Sequential rounds of RNA-dependent RNA transcription drive endogenous small-RNA biogenesis in the ERGO-1/Argonaute pathway. Proc. Natl. Acad. Sci. U. S. A. 107, 3582-3587. Abstract Article

Vastenhouw, N.L., Fischer, S.E., Robert, V.J., Thijssen, K.L., Fraser, A.G., Kamath, R.S., Ahringer, J., and Plasterk, R.H. (2003). A genome-wide screen identifies 27 genes involved in transposon silencing in C. elegans. Curr. Biol. 13, 1311-1316. Abstract Article

Vasu, S.K., and Forbes, D.J. (2001). Nuclear pores and nuclear assembly. Curr. Opin. Cell Biol. 13, 363-375. Abstract Article

Vought, V.E., Ohmachi, M., Lee, M.H., and Maine, E.M. (2005). EGO-1, a putative RNA-directed RNA polymerase, promotes germline proliferation in parallel with GLP-1/Notch signaling and regulates the spatial organization of nuclear pore complexes and germline P granules in Caenorhabditis elegans. Genetics 170, 1121-1132. Abstract Article

Wagner, E.J., Berkow, A., and Marzluff, W.F. (2005). Expression of an RNAi-resistant SLBP restores proper S-phase progression. Biochem. Soc. Trans. 33, 471-473. Abstract 
Wang, G., and Reinke, V. (2008). A C. elegans Piwi, PRG-1, regulates 21U-RNAs during spermatogenesis. Curr. Biol. 18, 861-867. Abstract Article

Warf, M.B., Shepherd, B.A., Johnson, W.E., and Bass, B.L. (2012). Effects of ADARs on small RNA processing pathways in C. elegans. Genome Res. 22, 1488-1498. Abstract Article

Welker, N.C., Habig, J.W., and Bass, B.L. (2007). Genes misregulated in C. elegans deficient in Dicer, RDE-4, or RDE-1 are enriched for innate immunity genes. RNA 13, 1090-1102. Abstract Article

Welker, N.C., Maity, T.S., Ye, X., Aruscavage, P.J., Krauchuk, A.A., Liu, Q., and Bass, B.L. (2011). Dicer's helicase domain discriminates dsRNA termini to promote an altered reaction mode. Mol. Cell 41, 589-599. Abstract Article

Welker, N.C., Pavelec, D.M., Nix, D.A., Duchaine, T.F., Kennedy, S., and Bass, B.L. (2010). Dicer's helicase domain is required for accumulation of some, but not all, C. elegans endogenous siRNAs. RNA 16, 893-903. Abstract Article

Wightman, B., Ha, I., and Ruvkun, G. (1993). Posttranscriptional regulation of the heterochronic gene lin-14 by lin-4 mediates temporal pattern formation in C. elegans. Cell 75, 855-862 Abstract Article

Yang, H., Zhang, Y., Vallandingham, J., Li, H., Florens, L., and Mak, H.Y. (2012). The RDE-10/RDE-11 complex triggers RNAi-induced mRNA degradation by association with target mRNA in C. elegans. Genes Dev. 26, 846-856. Abstract Article

Yigit, E., Batista, P.J., Bei, Y., Pang, K.M., Chen, C.C., Toila, N.H., Joshua-Tor, L., Mitani, S., Simard, M.J. and Mello, C.C. (2006). Analysis of the C. elegans Argonaute family reveals that distinct Argonautes act sequentially during RNAi. Cell 127, 747-757. Abstract Article

Zamore, P.D., Tuschl, T., Sharp, P.A., and Bartel, D.P. (2000). RNAi, double-stranded RNA directs the ATP-dependent cleavage of mRNA at 21 to 23 nucleotide intervals. Cell 101, 25-33. Abstract Article

Zhang, C., Montgomery, T.A., Fischer, S.E., Garcia, S.M., Riedel, C.G., Fahlgren, N., Sullivan, C., Carrington, J.C., and Ruvkun, G. (2012). The Caenorhabditis elegans RDE-10/RDE-11 complex regulates RNAi by promoting secondary siRNA amplification. Curr. Biol. 22, 881-890. Abstract Article

Zhang, C., Montgomery, T.A., Gabel, H.W., Fischer, S.E., Phillips, C.M., Fahlgren, N., Sullivan, C.M., Carrington, J.C., and Ruvkun, G. (2011). mut-16 and other mutator class genes modulate 22G and 26G siRNA pathways in Caenorhabditis elegans. Proc. Natl. Acad. Sci. U. S. A. 108, 1201-1208. Abstract Article

Zhuang, J.J., and Hunter, C.P. (2011). Tissue specificity of Caenorhabditis elegans enhanced RNA interference mutants. Genetics 188, 235-237. Abstract Article

Zhuang, J.J., and Hunter, C.P. (2012). RNA interference in Caenorhabditis elegans, uptake, mechanism, and regulation. Parasitology 139, 560-573. Abstract Article

Zipperlen, P., Fraser, A.G., Kamath, R.S., Martinez-Campos, M., and Ahringer, J. (2001). Roles for 147 embryonic lethal genes on C. elegans chromosome I identified by RNA interference and video microscopy. EMBO J. 20, 3984-3992. Abstract Article

\begin{tabular}{|c|l}
\hline @) & All WormBook content, except \\
SODMERIGHIS RESERVED & Commons Attribution License.
\end{tabular} 